\title{
Single-Pot Asymmetric Approach toward Enantioenriched Quaternary Stereocenter-Containing Alkylidenecyclobutanes
}

Michael Eisold, Gabriel M. Kiefl, Dorian Didier*

Department Chemie, Ludwig-Maximillians-Universität

Butenandtestrasse 5-13, D-81377 Munich

dorian.didier@cup.uni-muenchen.de

Supporting Information

1. General considerations 2

2. Experimental procedures 4

a) General procedures 4

b) Experimental data $\quad 7$

3. NMR spectra 19

4. Asymmetric Part

a) Experimental data

b) Chiral HPLC chromatograms

5. Representative chromatograms for diastereoisomeric ratios determination 49 


\section{General considerations}

Commercial available starting materials were used without further purification unless otherwise stated. All reactions were carried out under $\mathrm{N}_{2}$ atmosphere in flame-dried glassware. Syringes which were used to transfer anhydrous solvents or reagents were purged with nitrogen prior to use.

$\mathrm{CH}_{2} \mathrm{Cl}_{2}$ was predried over $\mathrm{CaCl}_{2}$ and distilled from $\mathrm{CaH}_{2}$. THF was refluxed and distilled from sodium benzophenone ketyl under nitrogen. $\mathrm{Et}_{2} \mathrm{O}$ was predried over $\mathrm{CaCl}_{2}$ and passed through activated $\mathrm{Al}_{2} \mathrm{O}_{3}$ (the solvent purification system SPS-400-2 from Innovative Technologies Inc.).

Chromatography purifications were performed using silica gel $\left(\mathrm{SiO}_{2}, 0.040-0.063 \mathrm{~mm}, 230-400\right.$ mesh ASTM) from Merck or Florisil ( $\mathrm{MgSiO}_{3}, 60-100$ mesh) from APOLLO. The spots were visualized under UV $(254 \mathrm{~nm})$ or by staining the TLC plate with $\mathrm{KMnO}_{4}$ solution $\left(\mathrm{K}_{2} \mathrm{CO}_{3}, 10 \mathrm{~g}-\mathrm{KmnO}_{4}, 1.5 \mathrm{~g}-\mathrm{H}_{2} \mathrm{O}, 150 \mathrm{~mL}-\right.$ $\mathrm{NaOH} 10 \%$ in $\mathrm{H}_{2} \mathrm{O}, 1.25 \mathrm{~mL}$ ), p-anisaldehyde solution (conc. $\mathrm{H}_{2} \mathrm{SO}_{4}, 10 \mathrm{~mL}-\mathrm{EtOH}, 200 \mathrm{~mL}-\mathrm{AcOH}, 3 \mathrm{~mL}$ - $p$-anisaldehyde, $4 \mathrm{~mL}$ ) and/or "Magic stain" (phosphomolybdic acid, $2.5 \mathrm{~g}-\mathrm{Ce}\left(\mathrm{SO}_{4}\right)_{2}, 1 \mathrm{~g}-$ conc. $\mathrm{H}_{2} \mathrm{SO}_{4}$, $6 \mathrm{~mL}-\mathrm{H}_{2} \mathrm{O}, 94 \mathrm{~mL}$ ).

Diastereoisomeric ratios were determined by ${ }^{1} \mathrm{H}$ NMR and ${ }^{13} \mathrm{C}$ NMR. NMR spectra were recorded on Bruker WH-400 instrument. Chemical shifts are reported as $\delta$ values in ppm relative to residual solvent peak $\left({ }^{1} \mathrm{H}-\right.$ NMR) or solvent peak $\left({ }^{13} \mathrm{C}-\mathrm{NMR}\right)$ in deuterated chloroform $\left(\mathrm{CDCl}_{3}: \delta 7.26 \mathrm{ppm}\right.$ for ${ }^{1} \mathrm{H}-\mathrm{NMR}$ and $\delta 77.16$ ppm for ${ }^{13} \mathrm{C}-\mathrm{NMR}$ ). Abbreviations for signal coupling are as follows: s (singlet), $\mathrm{d}$ (doublet), $\mathrm{t}$ (triplet), $\mathrm{q}$ (quartet), quint (quintet), m (multiplet) and br (broad). Reaction endpoints were determined by GC monitoring of the reactions. Gas chromatography was performed with machines of Agilent Technologies 7890, using a column of type HP 5 (Agilent 5\% phenylmethylpolysiloxane; length: $15 \mathrm{~m}$; diameter: 0.25 mm; film thickness: $0.25 \mu \mathrm{m}$ ) or Hewlett-Packard 6890 or 5890 series II, using a column of type HP 5 (HewlettPackard, 5\% phenylmethylpolysiloxane; length: $15 \mathrm{~m}$; diameter: 0,25 mm; film thickness: $0.25 \mu \mathrm{m}$ ). High resolution mass spectra (HRMS) and low resolution mass spectra (LRMS) were recorded on Finnigan MAT 95Q or Finnigan MAT 90 instrument or JEOL JMS-700. Infrared spectra were recorded on a Perkin 281 IR spectrometer and samples were measured neat (ATR, Smiths Detection DuraSample IR II Diamond ATR). The absorption bands were reported in wave numbers $\left(\mathrm{cm}^{-1}\right)$ and abbreviations for intensity are as follows: vs (very strong; maximum intensity), s (strong; above $75 \%$ of max. intensity), m (medium; from $50 \%$ to $75 \%$ of max. intensity), w (weak; below $50 \%$ of max. intensity) and br (broad). Melting points were determined on a Büchi B-540 apparatus and uncorrected. Optical rotation values were determined on a P8000-P8100-T polarimeter from A. Krüss Optronic, running software V3.0 with $5 \mathrm{~cm}$ path length. Enantiomeric excess was determined using a Shimadzu prominence HPLC machine running LabSolutions V5.42SP5 equipped with Chiralcel Technologies Europe columns with $0.46 \mathrm{~cm}$ diameter and $25 \mathrm{~cm}$ length, from Daicel chemical industries LTD.

$[n$-BuLi $]=2.41 \mathrm{M}$ in hexane (titration with isopropanol / 1,10-phenanthroline), purchased from Rockwood Lithium GmbH.

$\left[\mathrm{Me}_{3} \mathrm{Al}\right]=2.00 \mathrm{M}$ in hexane (considered from commercial source). 
[Allylzinc bromide $\mathrm{LiCl}]=0.77 \mathrm{M}$ in THF (titration with iodine) .

[(2-Methylallyl)zinc bromide $\mathrm{LiCl}]=0.53 \mathrm{M}$ in THF (titration with iodine).

$[i$-Propylmagnesium bromide $\mathrm{LiCl}]=1.26 \mathrm{M}$ in THF (titration with iodine).

[Methylmagnesium chloride] $=2.73 \mathrm{M}$ in THF (titration with iodine).

[Ethylmagnesium bromide] $=1.25 \mathrm{M}$ in THF (titration with iodine) .

[Cyclopropylmagnesium bromide $\mathrm{LiCl}]=0.87 \mathrm{M}$ in THF (titration with iodine).

[Phenethylmagnesium bromide $\mathrm{LiCl}]=0.86 \mathrm{M}$ in THF (titration with iodine).

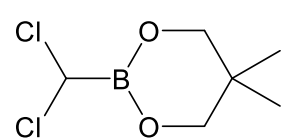

$1 \mathbf{b}$<smiles>ClC(Cl)B1O[C@@H](Cl)[C@@H](Cl)O1</smiles>

$(R, R)-5$<smiles>ClC(Cl)B1O[C@@H](Cl)[C@@H](Cl)O1</smiles>

$(S, S)-5$<smiles>CCCCCCCCCCCCC</smiles>

$(R, R)-8$

The preparation of $\mathbf{1 b},{ }^{1}(\boldsymbol{R}, \boldsymbol{R})-\mathbf{5}^{2},(\boldsymbol{S}, \boldsymbol{S})-\mathbf{5}^{3}$ and $(\boldsymbol{R}, \boldsymbol{R})-\mathbf{8}^{4}$ was done according to known literature procedures.

${ }^{1}$ P. G. M. Wuts, P. A. Thompson, J. Organomet. Chem., 1982, 234, 137-41.

${ }^{2}$ R. W. Hoffmann, K. Ditrich, G. Köster, R. Stürmer, Chem. Ber., 1989, 122, 1783-1789.

${ }^{3}$ L. Li, S. Zhao, A. Joshi-Pangu, M. Diane, M. Biscoe, J. Am. Chem. Soc., 2014, 136, 14027-14030.

${ }^{4}$ D. S. Matteson, H.-W. Man, J. Org. Chem., 1994, 59, 5734-5741. 


\section{Experimental procedures}

\section{a) General procedures}

General Procedure A:
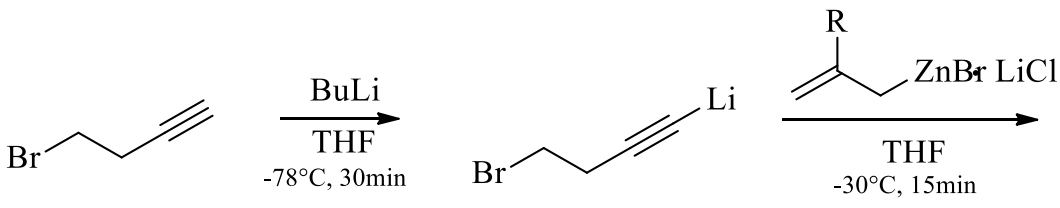

r.t., $1 \mathrm{~h}$

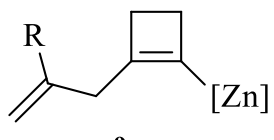

9a

To a solution of 4-bromobut-1-yne in THF ( $0.5 \mathrm{M})$ was added dropwise a solution of $n$-butyllithium (1.0 equiv) in hexane at $-78{ }^{\circ} \mathrm{C}$. After stirring for 30 minutes at aforesaid temperature, the solution was warmed to $-30{ }^{\circ} \mathrm{C}$ and stirred for an additional 5 minutes. A solution of the zinc organyl with $\mathrm{R}=\mathrm{H}$, Me (1.0 equiv) in THF was added slowly and the solution was stirred for another 15 mins at $-30{ }^{\circ} \mathrm{C}$. The mixture was warmed to room temperature and allowed to react for $60 \mathrm{mins}$, to yield the metallated cyclobutenyl derivative 9a.
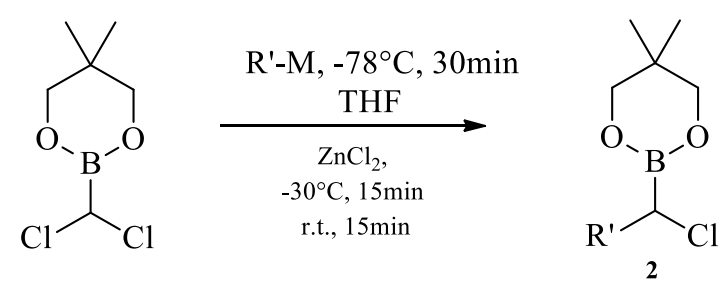

A second flask was charged with a solution of the dichloromethylboronic ester in THF ( $0.5 \mathrm{M})$ and a solution of the lithium organyl and Grignard species (1.0 equiv), respectively, was added dropwise at $-78{ }^{\circ} \mathrm{C}$. After stirring for 30 minutes at aforesaid temperature, a solution of zinc chloride (1.0 M, 1.0 equiv) in THF was added. The resulting mixture was then stirred for 15 minutes at $-30{ }^{\circ} \mathrm{C}$ and finally for another 15 minutes at room temperature to yield the homologated alkylboronic ester $\mathbf{2}$.<smiles>[R]C(=C)CC1=C([Y10])CC1</smiles>

9a

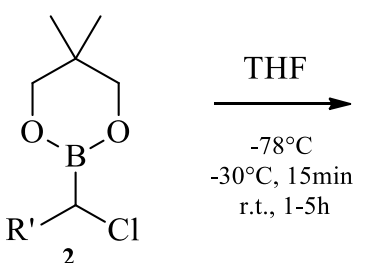<smiles>[R]C(=C)CC1=C(C([R])B2OCC(C)(C)CO2)CC1</smiles>

3

The mixture containing the metallated cyclobutenyl derivative 9a was then added via syringe to the mixture of the homologated alkylboronic ester 2 at $-78{ }^{\circ} \mathrm{C}$. After stirring for 15 minutes at $-30{ }^{\circ} \mathrm{C}$, the resulting mixture was allowed to react for another $1-2 \mathrm{~h}$ at room temperature. Volatiles were removed under reduced pressure, to yield a pale yellow residue. The residue 3 was dissolved in $\mathrm{CH}_{2} \mathrm{Cl}_{2}(0.5 \mathrm{M})$ and cooled to $0{ }^{\circ} \mathrm{C}$.<smiles>[R]C(=C)CC1=C(C([R])B2OCC(C)(C)CO2)CC1</smiles>

3

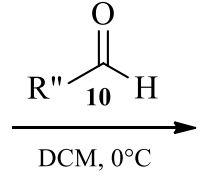


The liquid and solid aldehydes 10 ( 0.5 equiv) were added neat and dissolved in $\mathrm{CH}_{2} \mathrm{Cl}_{2}$, respectively. Upon full consumption of the boronate intermediate 3 , saturated ammonium chloride solution and $\mathrm{Et}_{2} \mathrm{O}$ were added and the mixture was stirred vigorously. The aqueous phase was extracted with $\mathrm{Et}_{2} \mathrm{O}(3 \times 20 \mathrm{~mL})$ and the combined organic layers washed with aqueous sodium metabisulfite $(20 \mathrm{~mL})$. The washed solution was dried over magnesium sulfate, filtrated and concentrated under reduced pressure. The crude alcohol was purified by flash-column chromatography on silica gel with ethyl acetate in hexanes and $\mathrm{Et}_{2} \mathrm{O}$ in hexanes, respectively, to afford the pure alkylidenecyclobutylcarbinols 4.

\section{$\underline{\text { General Procedure B: }}$}
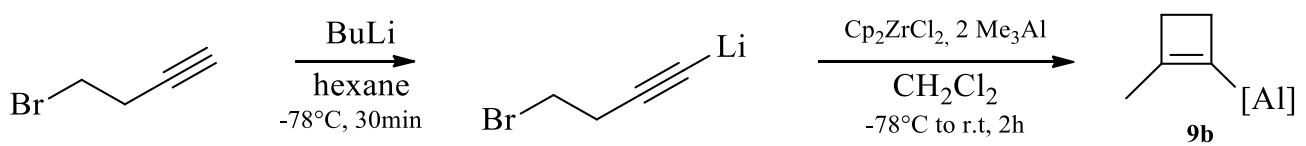

To a solution of 4-bromobutyne in hexane $(0.5 \mathrm{M})$, was added dropwise a solution of $n$-butyllithium (1.0 equiv) in hexane at $-78{ }^{\circ} \mathrm{C}$ and stirred for 30 minutes. A second flask was charged with $\mathrm{Cp}_{2} \mathrm{ZrCl}_{2}$ (1.0 equiv) in $\mathrm{CH}_{2} \mathrm{Cl}_{2}(0.5 \mathrm{M})$ and trimethylaluminium (2.0 equiv) was added at room temperature and stirred for 30 minutes. The second solution was transferred to the first one at $-78{ }^{\circ} \mathrm{C}$ via cannula. The resulting mixture was the allowed to stir at room temperature for 2 hours to form the metallated cyclobutenyl derivative $\mathbf{9 b}$.

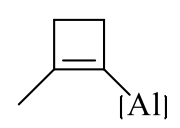

$9 \mathbf{b}$

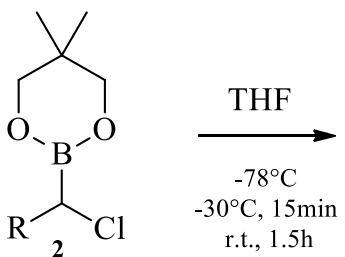

r.t., $1.5 \mathrm{~h}$

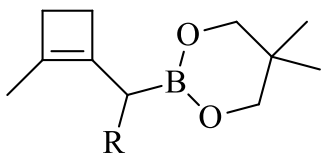

3

The homologated alkylboronic ester $\mathbf{2}$ was received following the second step of the general procedure A. The solution was added to the mixture containing the metallated cyclobutenyl derivative $9 \mathbf{b}$ at $-78^{\circ} \mathrm{C}$. The resulting mixture was allowed to react at room temperature for $1.5 \mathrm{~h}$. Volatiles were removed under reduced pressure, to yield a pale yellow residue. The residue 3 was dissolved in $\mathrm{CH}_{2} \mathrm{Cl}_{2}(0.5 \mathrm{M})$ and cooled to $0{ }^{\circ} \mathrm{C}$.
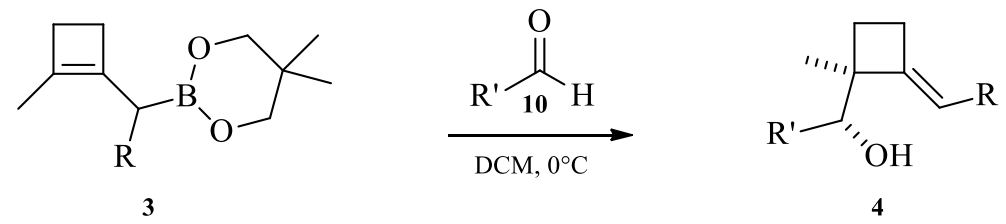

The liquid and solid aldehydes $\mathbf{1 0}$ ( 0.5 equiv) were added neat and dissolved in $\mathrm{CH}_{2} \mathrm{Cl}_{2}$, respectively. Upon full consumption of the boronate intermediate 3 , saturated ammonium chloride solution and $\mathrm{Et}_{2} \mathrm{O}$ were added and the mixture was stirred vigorously. The aqueous phase was extracted with $\mathrm{Et}_{2} \mathrm{O}(3 \times 20 \mathrm{~mL})$ and the combined organic layers washed with aqueous sodium metabisulfite $(20 \mathrm{~mL})$. The washed solution was dried over magnesium sulfate, filtrated and concentrated under reduced pressure. The crude alcohol was purified by flash-column chromatography on silica gel with ethyl acetate in hexanes and $\mathrm{Et}_{2} \mathrm{O}$ in hexanes, 
respectively, to afford the pure alkylidenecyclobutylcarbinols 4.

\section{General Procedure C:}<smiles>CC(C)OB(OC(C)C)C(O)C(O)C(O)C(F)(Cl)C(F)(Cl)Cl</smiles><smiles>ClC(Cl)B1OC(C2CCCCC2)C(C2CCCCC2)O1</smiles><smiles>[R]C(Cl)B1OC(C2CCCC2)C(C)C1[As]</smiles>

A flask was charged with a solution of diisopropyl (dichloromethyl)boronate in THF (0.5 M). A solution of the dicyclohexylethanediol in THF $(0.2 \mathrm{M})$ was added at room temperature and the mixture was stirred for 30 minutes to allow full conversion. The resulting solvent mixture was removed under reduced pressure and substituted for pure THF $(0.5 \mathrm{M})$. The solution was cooled to $-78{ }^{\circ} \mathrm{C}$ and a solution of the lithium organyl and Grignard species (1.0 equiv), respectively, was added dropwise. After stirring for 30 minutes at aforesaid temperature, a solution of zinc chloride (1.0 M, 1.0 equiv) in THF was added. The resulting mixture was then stirred for 15 minutes at $-30{ }^{\circ} \mathrm{C}$ and finally for another 15 minutes at room temperature to yield the homologated alkylboronic ester 6.

The pure alkylidenecyclobutylcarbinols $\mathbf{4}$ were afforded by following the third and fourth step of the general procedure A.

\section{General Procedure D:}

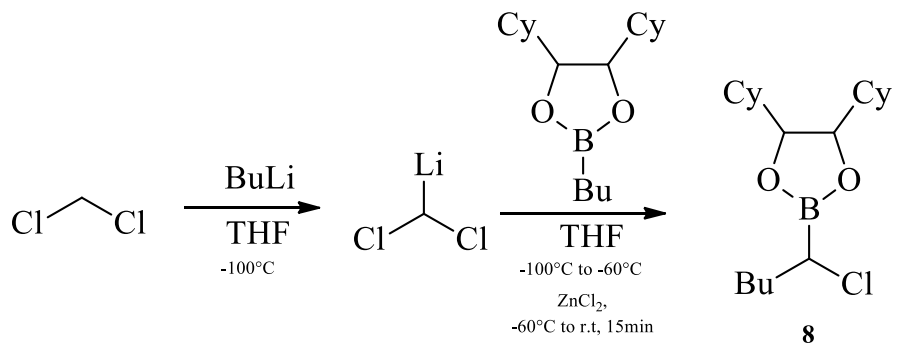

A second flask was charged with a solution of $\mathrm{CH}_{2} \mathrm{Cl}_{2}$ in THF $(0.5 \mathrm{M})$ and cooled to $-100{ }^{\circ} \mathrm{C}$. $n$-Butyllithium was added dropwise and the resulting solution was stirred for another 30 minutes at aforesaid temperature. (4R,5R)-2-butyl-4,5-dicyclohexyl-1,3,2-dioxaborolane in THF $(0.5 \mathrm{M})$ was added and the solution was allowed to warm to $-60{ }^{\circ} \mathrm{C}$, followed by the addition of zinc chloride (1.0 M, 1.0 equiv) in THF. The mixture was stirred for another 15 minutes at said temperature to yield the homologated alkylboronic ester 8 .

The pure alkylidenecyclobutylcarbinols $\mathbf{4}$ were afforded by following the third and fourth step of the general procedure A. 


\section{b) Experimental data}

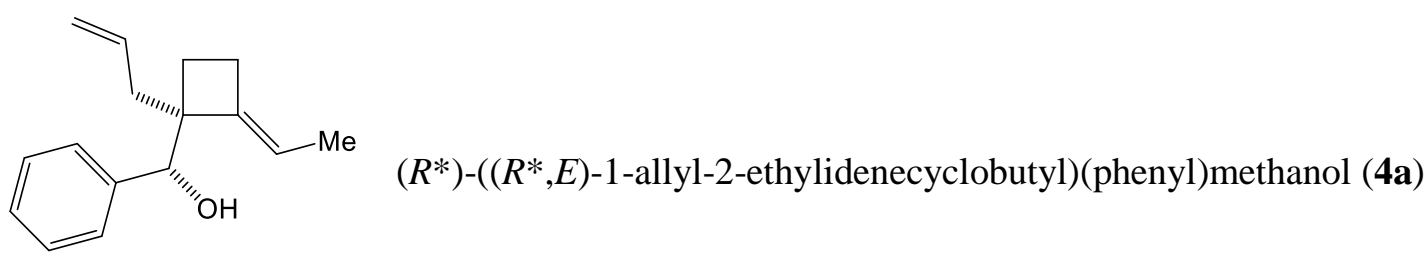

Using allylzinc bromide as the zinc organyl, methylmagnesium chloride as the Grignard species, 2(dichloromethyl)-5,5-dimethyl-1,3,2-dioxaborinane as the boronic ester and benzaldehyde according to general procedure A, provided $\mathbf{4 a}(60 \mathrm{mg}, 53 \%)$ as a colourless oil in 97:3 $d r$.

Colourless oil, $\boldsymbol{R}_{\mathbf{f}}=0.19\left(10: 1\right.$ hexane:EtOAc, UV, PAA). ${ }^{1} \mathbf{H}$ NMR $\left(400 \mathrm{MHz}, \mathrm{CDCl}_{3}\right): \delta(\mathrm{ppm}) 7.36-7.31$ $(\mathrm{m}, 2 \mathrm{H}), 7.29-7.18(\mathrm{~m}, 3 \mathrm{H}), 5.80(\mathrm{~m}, 1 \mathrm{H}), 5.35-5.20(\mathrm{~m}, 1 \mathrm{H}), 5.07-4.86(\mathrm{~m}, 2 \mathrm{H}), 4.71(\mathrm{~d}, J=2.6 \mathrm{~Hz}$, $1 \mathrm{H}), 2.35(\mathrm{~m}, 1 \mathrm{H}), 2.32-2.21(\mathrm{~m}, 2 \mathrm{H}), 2.18(\mathrm{~d}, J=3.0 \mathrm{~Hz}, 1 \mathrm{H}), 2.01-1.90(\mathrm{~m}, 2 \mathrm{H}), 1.75(\mathrm{~m}, 1 \mathrm{H}), 1.48(\mathrm{dt}$, $J=6.6,1.7 \mathrm{~Hz}, 3 \mathrm{H}) .{ }^{13} \mathrm{C}$ NMR $\left(101 \mathrm{MHz}, \mathrm{CDCl}_{3}\right): \delta(\mathrm{ppm}) 144.1,141.1,136.0,127.8,127.5,127.4,117.5$, 117.2, 78.1, 55.0, 38.2, 24.4, 23.4, 13.2. HRMS (EI): calcd for $\mathrm{C}_{16} \mathrm{H}_{20} \mathrm{O}^{+}[\mathrm{M}]^{+}: 228.1514$, found 228.1490. IR: v (cm-1) 3446 (br. vw), 3071 (vw), 3029 (vw), 2975 (w), 2931 (w), 2912 (w), 2855 (w), 1638 (w), 1602 (vw), 1494 (w), 1476 (vw), 1451 (w), 1438 (w), 1414 (w), 1376 (w), 1343 (w), 1323 (w), 1289 (w), 1243 (w), 1195 (w), 1156 (w), $1081(\mathrm{w}), 1062$ (w), 1035 (m), 1018 (m), 998 (m), 964 (w), 909 (s), $814(\mathrm{w}), 771$ (w), $732(\mathrm{~m}), 700(\mathrm{vs}), 686(\mathrm{~m}), 675(\mathrm{~m})$.

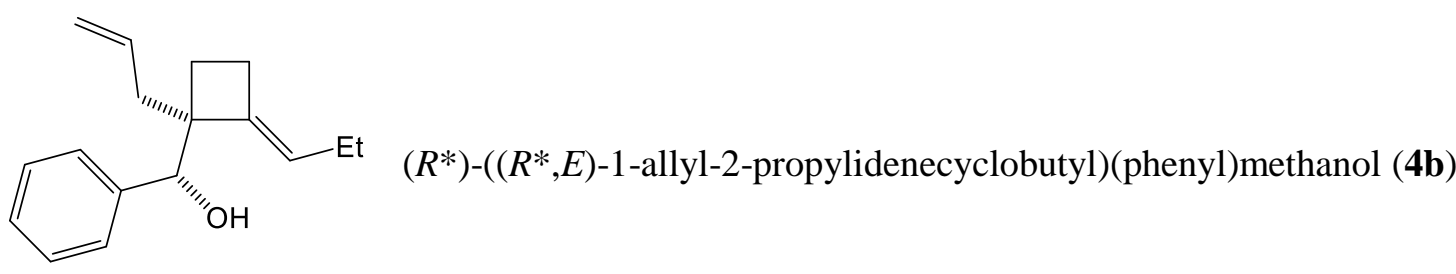

Using allylzinc bromide as the zinc organyl, ethylmagnesium chloride as the Grignard species, 2(dichloromethyl)-5,5-dimethyl-1,3,2-dioxaborinane as the boronic ester and benzaldehyde according to general procedure A, provided $\mathbf{4 b}(47 \mathrm{mg}, 65 \%)$ as a colourless oil in 97:3 $d r$.

Colourless oil, $\boldsymbol{R}_{\mathbf{f}}=0.15$ (50:1 hexane:EtOAc, UV, PAA). ${ }^{1} \mathbf{H}$ NMR $\left(400 \mathrm{MHz}, \mathrm{CDCl}_{3}\right): \delta(\mathrm{ppm}) 7.38-7.31$ $(\mathrm{m}, 2 \mathrm{H}), 7.30-7.10(\mathrm{~m}, 3 \mathrm{H}), 5.86-5.76(\mathrm{~m}, 1 \mathrm{H}), 5.26-5.20(\mathrm{~m}, 1 \mathrm{H}), 5.07-4.88(\mathrm{~m}, 2 \mathrm{H}), 4.71(\mathrm{~s}, 1 \mathrm{H})$, $2.41-2,32(\mathrm{~m}, 1 \mathrm{H}), 2.31-2.08(\mathrm{~m}, 3 \mathrm{H}), 2.03-1.92(\mathrm{~m}, 2 \mathrm{H}), 1.92-1.81(\mathrm{~m}, 2 \mathrm{H}), 1.80-1.70(\mathrm{~m}, 1 \mathrm{H})$, $0.90(\mathrm{t}, J=7.5 \mathrm{~Hz}, 3 \mathrm{H}) .{ }^{13} \mathrm{C} \mathbf{N M R}\left(101 \mathrm{MHz}, \mathrm{CDCl}_{3}\right): \delta(\mathrm{ppm}) 142.7,141.1,136.0,127.7,127.6,127.4$, 125.1, 117.2, 78.1, 54.8, 38.4, 24.5, 23.3, 21.4, 14.4. HRMS (EI): calcd for $\mathrm{C}_{17} \mathrm{H}_{22} \mathrm{O}^{+}[\mathrm{M}]^{+}: 242.1671$, found 242.1710. IR: $v\left(\mathrm{~cm}^{-1}\right) 3446$ (br. vw), 3071 (vw), 3029 (vw), 2959 (w), 2870 (w), 2358 (vw), 2336 (vw), 1636 (w), 1451 (m), 1436 (w), 1297 (w), 1195 (w), 1035 (m), 1022 (m), 1014 (m), 999 (m), 971 (w), 909 (m), 770 (w), 739 (m), 700 (vs). 


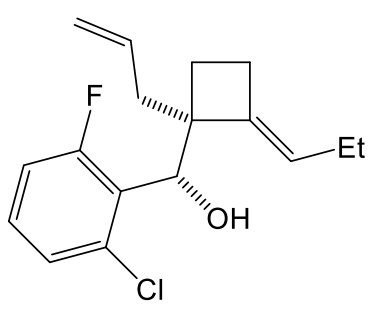

$\left(S^{*}\right)-\left(\left(R^{*}, E\right)-1\right.$-allyl-2-propylidenecyclobutyl)(2-chloro-6-fluorophenyl)methanol $(4 c)$

Using allylzinc bromide as the zinc organyl, ethylmagnesium chloride as the Grignard species, 2(dichloromethyl)-5,5-dimethyl-1,3,2-dioxaborinane as the boronic ester and 2-chloro-6-fluorobenzaldehyde according to general procedure A, provided $\mathbf{4 c}(90 \mathrm{mg}, 61 \%)$ as a colourless oil in 97:3 $d r$.

Colourless oil, $\boldsymbol{R}_{\mathbf{f}}=0.18$ (100:1 hexane:EtOAc, UV, PAA). ${ }^{1} \mathbf{H}$ NMR $\left(400 \mathrm{MHz}, \mathrm{CDCl}_{3}\right): \delta(\mathrm{ppm}) 7.23-$ $7.09(\mathrm{~m}, 2 \mathrm{H}), 7.06-6.89(\mathrm{~m}, 1 \mathrm{H}), 6.01-5.85(\mathrm{~m}, 1 \mathrm{H}), 5.45-5.16(\mathrm{~m}, 2 \mathrm{H}), 5.16-5.00(\mathrm{~m}, 2 \mathrm{H}), 2.82(\mathrm{dd}$, $J=9.9,6.5 \mathrm{~Hz}, 1 \mathrm{H}), 2.54-2.39(\mathrm{~m}, 1 \mathrm{H}), 2.40-2.11(\mathrm{~m}, 4 \mathrm{H}), 1.97-1.70(\mathrm{~m}, 3 \mathrm{H}), 0.88(\mathrm{t}, J=7.5 \mathrm{~Hz}, 3 \mathrm{H})$. ${ }^{13}$ C NMR (101 MHz, CDCl $): \delta(\mathrm{ppm})$ 163.2, 160.8, 140.3, 135.7, 134.8 (d, $\left.J=7.7 \mathrm{~Hz}\right), 129.1$ (d, $J=10.5$ Hz), $127.3(\mathrm{~d}, J=14.3 \mathrm{~Hz}), 126.0(\mathrm{dd}, J=9.0,2.6 \mathrm{~Hz}), 117.5,115.3(\mathrm{~d}, J=24.7 \mathrm{~Hz}), 75.4,56.1,39.8(\mathrm{~d}, J=$ $2.9 \mathrm{~Hz}), 24.6,23.6(\mathrm{~d}, J=3.7 \mathrm{~Hz}), 21.4,14.2$. HRMS (EI): calcd for $\mathrm{C}_{17} \mathrm{H}_{19} \mathrm{ClFO}^{+}[\mathrm{M}-\mathrm{H}]^{+}:$: 293.1108, found 293.1099. IR: v (cm-1) 3471 (br. vw), 3074 (vw), 2959 (m), 2929 (w), 2871 (w), 2359 (w), 2333 (w), 1603 (m), 1575 (m), 1451 (s), 1407 (w), 1243 (m), 1186 (m), 1139 (w), 1072 (w), 1029 (m), 998 (m), 909 (s), 893 (s), 781 (vs), $756(\mathrm{~m}), 720(\mathrm{~m})$.

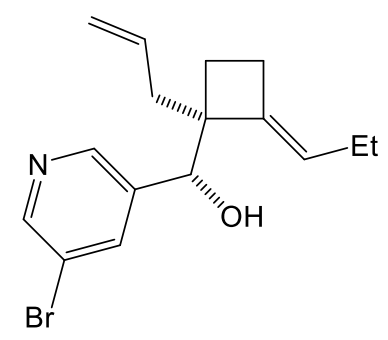

$\left(S^{*}\right)-\left(\left(R^{*}, E\right)\right.$-1-allyl-2-propylidenecyclobutyl)(5-bromopyridin-3-yl)methanol (4d)

Using allylzinc bromide as the zinc organyl, ethylmagnesium chloride as the Grignard species, 2(dichloromethyl)-5,5-dimethyl-1,3,2-dioxaborinane as the boronic ester and 5-bromo-3pyridinecarboxaldehyde according to general procedure A, provided $\mathbf{4 d}(98 \mathrm{mg}, 61 \%)$ as a colourless oil in 97:3dr.

Colourless oil, $\boldsymbol{R}_{\mathbf{f}}=0.17\left(10: 1\right.$ hexane:EtOAc, UV, PAA). ${ }^{1} \mathbf{H}$ NMR $\left(400 \mathrm{MHz}, \mathrm{CDCl}_{3}\right): \delta(\mathrm{ppm}) 8.50(\mathrm{~d}, J=$ $2.3 \mathrm{~Hz}, 1 \mathrm{H}), 8.46(\mathrm{~d}, J=1.9 \mathrm{~Hz}, 1 \mathrm{H}), 7.92(\mathrm{~s}, 1 \mathrm{H}), 5.96-5.80(\mathrm{~m}, 1 \mathrm{H}), 5.25-5.20(\mathrm{~m}, 1 \mathrm{H}), 5.15-4.94(\mathrm{~m}$, 2H), 4.74 (s, 1H), 3.10 (br. s, 1H), $2.55-2.27$ (m, 2H), 2.08 (m, 2H), $2.00-1.69$ (m, 4H), 0.94 (t, $J=7.5$ $\mathrm{Hz}, 3 \mathrm{H}) .{ }^{13} \mathrm{C}$ NMR (101 MHz, $\left.\mathrm{CDCl}_{3}\right): \delta(\mathrm{ppm}) 149.3,146.8,141.9,139.0,138.0,135.1,125.8,120.2$, 117.9, 74.9, 54.5, 39.3, 24.6, 22.9, 21.3, 14.3. HRMS (EI): calcd for $\mathrm{C}_{16} \mathrm{H}_{20} \mathrm{BrNO}^{+}[\mathrm{M}]^{+}:$321.0728, found 321.0728. IR: $v\left(\mathrm{~cm}^{-1}\right) 3248$ (br. w), 3074 (w), 2959 (m), 2928 (m), 2870 (m), 2851 (w), 2361 (w), 2340 (w), 1558 (m), 1456 (m), 1428 (m), 1419 (s), 1294 (m), 1223 (m), 1205 (m), 1139 (w), 1098 (m), 1048 (s), 1020 (s), 998 (m), 912 (vs), 885 (vs), 808 (w), 732 (s), 703 (vs). 


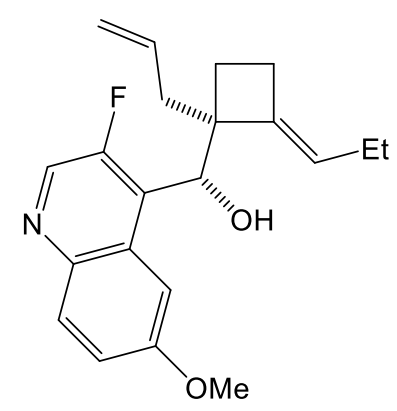

$\left(S^{*}\right)-\left(\left(R^{*}, E\right)-1\right.$-allyl-2-propylidenecyclobutyl)(3-fluoro-6-methoxyquinolin-4-yl)methanol (4e)

Using allylzinc bromide as the zinc organyl, ethylmagnesium chloride as the Grignard species, 2(dichloromethyl)-5,5-dimethyl-1,3,2-dioxaborinane as the boronic ester and 3-fluoro-6-methoxyquinoline-4carbaldehyde according to general procedure A, provided $4 \mathbf{e}(95 \mathrm{mg}, 56 \%)$ as a pale yellow solid in 97:3 $d r$. Pale yellow solid, $\mathrm{mp}=90-92^{\circ} \mathrm{C}, \boldsymbol{R}_{\mathbf{f}}=0.16\left(10: 1\right.$ hexane:EtOAc, UV, PAA). ${ }^{1} \mathbf{H}$ NMR $(400 \mathrm{MHz}$, $\left.\mathrm{CDCl}_{3}\right): \delta(\mathrm{ppm}) 8.49(\mathrm{~d}, J=2.2 \mathrm{~Hz}, 1 \mathrm{H}), 8.01-7.73(\mathrm{~m}, 2 \mathrm{H}), 7.25(\mathrm{dd}, J=9.2,2.8 \mathrm{~Hz}, 1 \mathrm{H}), 5.98-5.84$ $(\mathrm{m}, 1 \mathrm{H}), 5.60(\mathrm{~s}, 1 \mathrm{H}), 5.30-5.21(\mathrm{~m}, 1 \mathrm{H}), 5.18-4.87(\mathrm{~m}, 2 \mathrm{H}), 3.90(\mathrm{~s}, 3 \mathrm{H}), 3.15$ (br. s, 1H), $2.63(\mathrm{ddt}, J=$ 13.8, 6.4, $1.5 \mathrm{~Hz}, 1 \mathrm{H}), 2.42-2.11(\mathrm{~m}, 4 \mathrm{H}), 1.98-1.59(\mathrm{~m}, 3 \mathrm{H}), 0.79(\mathrm{t}, J=7.5 \mathrm{~Hz}, 3 \mathrm{H}) .{ }^{13} \mathbf{C} \mathbf{N M R}(101$ $\left.\mathrm{MHz}, \mathrm{CDCl}_{3}\right): \delta(\mathrm{ppm}) 157.7,155.8,153.3,142.1(\mathrm{~d}, J=2.4 \mathrm{~Hz}), 141.2,138.2(\mathrm{~d}, J=31.8 \mathrm{~Hz}), 135.5$, 131.1, $129.30-128.67(\mathrm{~m}), 125.7(\mathrm{~d}, J=1.5 \mathrm{~Hz}), 120.7$ (d, $J=2.5 \mathrm{~Hz}), 117.9,105.8$ (d, $J=5.2 \mathrm{~Hz}), 72.6(\mathrm{~d}$, $J=1.1 \mathrm{~Hz}), 56.2,55.6,39.9,24.6,23.6(\mathrm{~d}, J=3.5 \mathrm{~Hz}), 21.3,14.0$. HRMS (EI): calcd for $\mathrm{C}_{21} \mathrm{H}_{24} \mathrm{FNO}_{2}{ }^{+}[\mathrm{M}]^{+}$: 341.1791, found 341.1785. IR: $v\left(\mathrm{~cm}^{-1}\right) 3223$ (br. w), 3075 (w), 3005 (w), 2995 (w), 2963 (m), $2927(w)$, 2907 (w), 2831 (w), 2359 (w), 2337 (vw), 1622 (m), 1510 (s), 1465 (m), 1461 (w), 1426 (m), 1352 (m), 1306 (m), 1224 (vs), 1172 (w), 1137 (m), 1087 (s), 1046 (m), 1029 (s), 997 (m), 920 (s), 907 (s), 859 (m), 830 (vs), $794(\mathrm{~s}), 782(\mathrm{~m}), 709$ (m), $689(\mathrm{~m}), 677(\mathrm{~m})$.

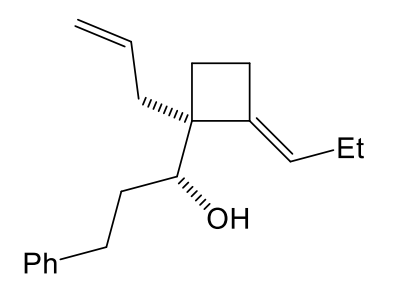

$\left(R^{*}\right)-1-\left(\left(R^{*}, E\right)-1-\right.$ allyl-2-propylidenecyclobutyl)-3-phenylpropan-1-ol (4f)

Using allylzinc bromide as the zinc organyl, ethylmagnesium chloride as the Grignard species, 2(dichloromethyl)-5,5-dimethyl-1,3,2-dioxaborinane as the boronic ester and 3-phenylpropanal according to general procedure A, provided $\mathbf{4 f}(65 \mathrm{mg}, 48 \%)$ as a colourless oil in 99:1 $d r$.

Colourless oil, $\boldsymbol{R}_{\mathbf{f}}=0.15$ (100:1 hexane:EtOAc, UV, PAA). ${ }^{1} \mathbf{H}$ NMR (400 MHz, $\left.\mathrm{CDCl}_{3}\right): \delta(\mathrm{ppm}) 7.38-$ $7.14(\mathrm{~m}, 5 \mathrm{H}), 6.02-5.73(\mathrm{~m}, 1 \mathrm{H}), 5.30-5.22(\mathrm{~m}, 1 \mathrm{H}), 5.10-5.06(\mathrm{~m}, 1 \mathrm{H}), 5.06-5.04(\mathrm{~m}, 1 \mathrm{H}), 3.63-$ $3.52(\mathrm{~m}, 1 \mathrm{H}), 3.05-2.93(\mathrm{~m}, 1 \mathrm{H}), 2.71-2.61(\mathrm{~m}, 1 \mathrm{H}), 2.50-2.38(\mathrm{~m}, 3 \mathrm{H}), 2.20-2.11(\mathrm{~m}, 1 \mathrm{H}), 2.04-1.88$ (m, 2H), $1.88-1.59(\mathrm{~m}, 5 \mathrm{H}), 0.98(\mathrm{t}, J=7.5 \mathrm{~Hz}, 3 \mathrm{H}) .{ }^{13} \mathbf{C} \mathbf{N M R}\left(101 \mathrm{MHz}, \mathrm{CDCl}_{3}\right): \delta(\mathrm{ppm}) 143.1,142.5$, 135.9, 128.6, 128.5, 125.9, 124.4, 117.0, 75.7, 54.3, 37.9, 33.5, 33.3, 24.3, 23.7, 21.3, 14.5. HRMS (EI): calcd for $\mathrm{C}_{19} \mathrm{H}_{26} \mathrm{O}^{+}[\mathrm{M}]^{+}:$270.1984, found 270.1995. IR: $v\left(\mathrm{~cm}^{-1}\right) 3453$ (br. vw), 3063 (vw), 3025 (w), 2957 (m), 2925 (w), 2869 (w), 2359 (w), 2336 (w), 1635 (w), 1496 (w), 1455 (m), $1436(\mathrm{w}), 1419$ (w), $1297(\mathrm{w})$, 1254 (w), 1157 (w), 1063 (m), 1030 (m), 998 (m), 910 (s), 849 (w), 747 (m), 698 (vs). 


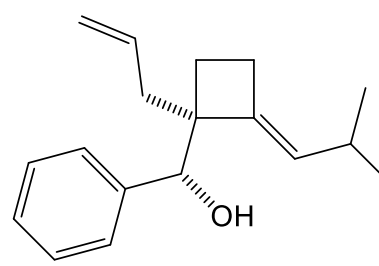

$\left(R^{*}\right)-\left(\left(R^{*}, E\right)-1-a l l y l-2-(2-m e t h y l p r o p y l i d e n e) c y c l o b u t y l\right)($ phenyl)methanol (4g)

Using allylzinc bromide as the zinc organyl, isopropylmagnesium chloride as the Grignard species, 2(dichloromethyl)-5,5-dimethyl-1,3,2-dioxaborinane as the boronic ester and benzaldehyde according to general procedure A, provided $\mathbf{4 g}(74 \mathrm{mg}, 73 \%)$ as a colourless oil in 99:1 $d r$.

Colourless oil, $\boldsymbol{R}_{\mathbf{f}}=0.12$ (100:1 hexane:EtOAc, UV, PAA). ${ }^{1} \mathbf{H}$ NMR $\left(400 \mathrm{MHz}, \mathrm{CDCl}_{3}\right): \delta(\mathrm{ppm}) 7.38-7.31$ (m, 2H), $7.29-7.17(\mathrm{~m}, 3 \mathrm{H}), 5.92-5.76(\mathrm{~m}, 1 \mathrm{H}), 5.09-5.03(\mathrm{~m}, 1 \mathrm{H}), 5.03-4.93(\mathrm{~m}, 2 \mathrm{H}), 4.70(\mathrm{~s}, 1 \mathrm{H})$, $2.41-2.33(\mathrm{~m}, 1 \mathrm{H}), 2.32-2.09(\mathrm{~m}, 4 \mathrm{H}), 2.06-1.92(\mathrm{~m}, 2 \mathrm{H}), 1.80-1.70(\mathrm{~m}, 1 \mathrm{H}), 0.90(\mathrm{dd}, J=6.7,3.6 \mathrm{~Hz}$, 6H). ${ }^{13} \mathrm{C}$ NMR (101 MHz, $\left.\mathrm{CDCl}_{3}\right): \delta$ (ppm) 141.1, 141.0, 135.9, 130.7, 127.7, 127.6, 127.4, 117.3, 78.0, 54.7, 38.8, 27.9, 24.7, 23.2, 23.1, 23.1. HRMS (EI): calcd for $\mathrm{C}_{18} \mathrm{H}_{24} \mathrm{O}^{+}[\mathrm{M}]^{+}:$: 256,1827, found 256.1792. IR: $v\left(\mathrm{~cm}^{-1}\right) 3436$ (br. vw), $3072(\mathrm{vw}), 3030$ (vw), 2952 (m), 2923 (w), 2865 (w), 1638 (w), $1494(\mathrm{w}), 1460$ (w), $1451(w), 1414(w), 1380(w), 1360(w), 1287(w), 1252$ (w), $1176(w), 1081(w), 1036(m), 1030(w)$, 1019 (m), 999 (m), 909 (s), 783 (w), 771 (vw), 753 (m), 741 (m), 701 (vs).

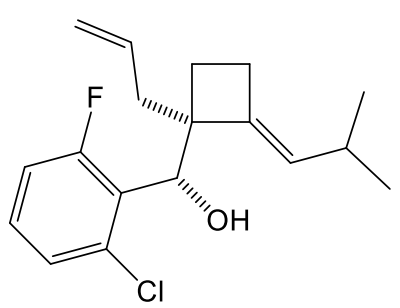

$\left(S^{*}\right)-\left(\left(R^{*}, E\right)-1\right.$-allyl-2-(2-methylpropylidene)cyclobutyl)(2-chloro-6fluorophenyl)-methanol (4h)

Using allylzinc bromide as the zinc organyl, isopropylmagnesium chloride as the Grignard species, 2(dichloromethyl)-5,5-dimethyl-1,3,2-dioxaborinane as the boronic ester and 2-chloro-6-fluorobenzaldehyde according to general procedure $\mathbf{A}$, provided $\mathbf{4 h}(94 \mathrm{mg}, 61 \%)$ as a colourless oil in 99:1 $d r$.

Colourless oil, $\boldsymbol{R}_{\mathbf{f}}=0.18$ (100:1 hexane:EtOAc, UV, PAA). ${ }^{1} \mathbf{H}$ NMR (400 MHz, $\left.\mathrm{CDCl}_{3}\right): \delta(\mathrm{ppm}) 7.25-$ $7.10(\mathrm{~m}, 2 \mathrm{H}), 7.04-6.92(\mathrm{~m}, 1 \mathrm{H}), 6.01-5.81(\mathrm{~m}, 1 \mathrm{H}), 5.31-5.25(\mathrm{~m}, 1 \mathrm{H}), 5.19-5.13(\mathrm{~m}, 1 \mathrm{H}), 5.11-$ $5.08(\mathrm{~m}, 1 \mathrm{H}), 5.08-5.03(\mathrm{~m}, 1 \mathrm{H}), 2.81(\mathrm{dd}, J=10.0,6.6 \mathrm{~Hz}, 1 \mathrm{H}), 2.50-2.40(\mathrm{~m}, 1 \mathrm{H}), 2.38-2.11(\mathrm{~m}, 5 \mathrm{H})$, $1.91-1.77(\mathrm{~m}, 1 \mathrm{H}), 0.91(\mathrm{~d}, J=6.7 \mathrm{~Hz}, 3 \mathrm{H}), 0.84(\mathrm{~d}, J=6.7 \mathrm{~Hz}, 3 \mathrm{H}) .{ }^{13} \mathbf{C}$ NMR $\left(101 \mathrm{MHz}, \mathrm{CDCl}_{3}\right): \delta$ (ppm) 163.2, 160.8, 138.5, 135.7, 134.8 (d, $J=7.7 \mathrm{~Hz}), 131.6$ (d, $J=1.9 \mathrm{~Hz}), 129.1$ (d, $J=10.5 \mathrm{~Hz}), 127.4$ $(\mathrm{d}, J=14.3 \mathrm{~Hz}), 126.0(\mathrm{~d}, J=3.1 \mathrm{~Hz}), 117.6,115.2(\mathrm{~d}, J=24.8 \mathrm{~Hz}), 75.4,56.1,40.3(\mathrm{~d}, J=2.4 \mathrm{~Hz}), 27.8$, 24.8, 23.6 (d, $J=4.2 \mathrm{~Hz}), 22.89$ (d, $J=18.3 \mathrm{~Hz}$ ). LRMS (DEP/EI) $m / z: 159.0\left[\mathrm{C}_{7} \mathrm{H}_{5} \mathrm{ClFO}\right]^{+}, 149.1\left[\mathrm{C}_{11} \mathrm{H}_{17}\right]^{+}$. HRMS (EI): calcd for $\mathrm{C}_{11} \mathrm{H}_{17}{ }^{+}\left[\mathrm{M}-\mathrm{C}_{7} \mathrm{H}_{5} \mathrm{ClFO}\right]^{+}:$149.1330, found 149.1307. IR: $v$ (cm $\left.{ }^{-1}\right) 3474$ (br. vw), 3076 (vw), 2955 (m), 2925 (m), 2867 (w), 1639 (w), 1604 (m), 1573 (m), 1452 (s), 1408 (w), 1284 (w), 1242 (m), 1187 (m), 1140 (w), 1101 (w), 1088 (w), 1072 (m), 1065 (w), 1028 (s), 999 (m), 955 (w), 910 (s), 893 (vs), 785 (vs), 773 (s), 728 (m), $700(\mathrm{~m})$. 


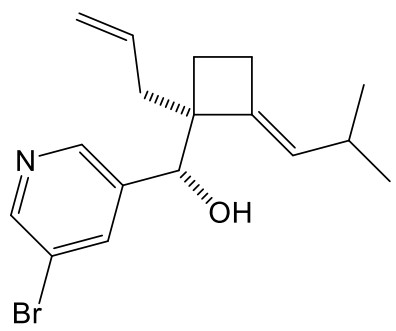

$\left(S^{*}\right)-\left(\left(R^{*}, E\right)-1\right.$-allyl-2-(2-methylpropylidene)cyclobutyl)(5-bromopyridin-3-yl)-

methanol (4i)

Using allylzinc bromide as the zinc organyl, isopropylmagnesium chloride as the Grignard species, 2(dichloromethyl)-5,5-dimethyl-1,3,2-dioxaborinane as the boronic ester and 5-bromo-3pyridinecarboxaldehyde according to general procedure $\mathbf{A}$, provided $\mathbf{4 i}(105 \mathrm{mg}, 62 \%)$ as a colourless oil in 99:1 dr.

Colourless oil, $\boldsymbol{R}_{\mathbf{f}}=0.17$ (10:1 hexane:EtOAc, UV, PAA). ${ }^{1} \mathbf{H}$ NMR $\left(400 \mathrm{MHz}, \mathrm{CDCl}_{3}\right): \delta(\mathrm{ppm}) 8.52(\mathrm{~d}, J=$ $2.3 \mathrm{~Hz}, 1 \mathrm{H}), 8.48(\mathrm{~d}, J=1.9 \mathrm{~Hz}, 1 \mathrm{H}), 8.05-7.78(\mathrm{~m}, 1 \mathrm{H}), 5.94-5.82(\mathrm{~m}, 1 \mathrm{H}), 5.15-5.09(\mathrm{~m}, 3 \mathrm{H}), 4.73(\mathrm{~s}$, 1H), 2.79 (br. s, 1H), $2.48-2.29$ (m, 2H), $2.29-2.18(\mathrm{~m}, 1 \mathrm{H}), 2.14-1.90(\mathrm{~m}, 3 \mathrm{H}), 1.87-1.78(\mathrm{~m}, 1 \mathrm{H})$, $0.94(\mathrm{t}, J=6.9 \mathrm{~Hz}, 6 \mathrm{H}) .{ }^{13} \mathbf{C} \mathbf{N M R}\left(101 \mathrm{MHz}, \mathrm{CDCl}_{3}\right): \delta(\mathrm{ppm}) 149.6,147.0,140.2,138.9,138.0,135.0$, 131.5, 120.1 118.0, 75.0, 54.4, 39.7, 27.9, 24.8, 23.1, 23.0, 22.9. HRMS (EI): calcd for $\mathrm{C}_{17} \mathrm{H}_{22} \mathrm{BrNO}^{+}[\mathrm{M}]^{+}$: 335.0885, found 335.0868. IR: $v\left(\mathrm{~cm}^{-1}\right) 3237$ (br. w), 3075 (w), 2954 (s), 2924 (m), 2866 (m), 1639 (w), 1581 (w), 1559 (w), 1464 (m), 1420 (s), 1381 (m), 1361 (m), 1292 (m), 1223 (m), 1206 (m), 1139 (m), 1099 (s), 1048 (s), 1021 (s), 999 (m), 912 (vs), 885 (s), 783 (m), 731 (vs), 703 (vs).

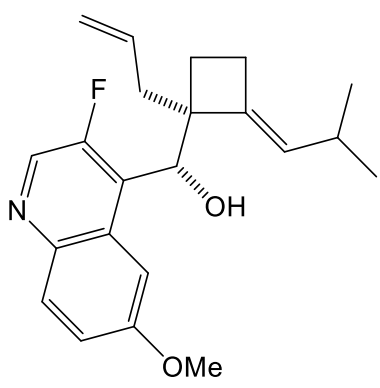

$\left(S^{*}\right)-\left(\left(R^{*}, E\right)-1-\right.$ allyl-2-(2-methylpropylidene)cyclobutyl)(3-fluoro-6methoxyquinolin-4-yl)methanol (4j)

Using allylzinc bromide as the zinc organyl, isopropylmagnesium chloride as the Grignard species, 2(dichloromethyl)-5,5-dimethyl-1,3,2-dioxaborinane as the boronic ester and 3-fluoro-6-methoxyquinoline-4carbaldehyde according to general procedure $\mathbf{A}$, provided $\mathbf{4 j}$ (107 $\mathrm{mg}, 60 \%)$ as a colourless oil in 99:1 $d r$. Colourless oil, $\boldsymbol{R}_{\mathbf{f}}=0.15\left(10: 1\right.$ hexane:EtOAc, UV, PAA). ${ }^{1} \mathbf{H}$ NMR $\left(400 \mathrm{MHz}, \mathrm{CDCl}_{3}\right): \delta(\mathrm{ppm}) 8.64-8.34$ $(\mathrm{m}, 1 \mathrm{H}), 7.96-7.86(\mathrm{~m}, 2 \mathrm{H}), 7.36-7.12(\mathrm{~m}, 1 \mathrm{H}), 5.60-5.84(\mathrm{~m}, 1 \mathrm{H}), 5.61-5.55(\mathrm{~m}, 1 \mathrm{H}), 5.14-4.99(\mathrm{~m}$, 3H), $3.99-3.79$ (m, 3H), $3.37-3.03(\mathrm{~m}, 1 \mathrm{H}), 2.62$ (dd, $J=13.1,6.3 \mathrm{~Hz}, 1 \mathrm{H}), 2.40-2.03(\mathrm{~m}, 5 \mathrm{H}), 1.90-$ $1.76(\mathrm{~m}, 1 \mathrm{H}), 0.87(\mathrm{dd}, J=6.8,1.2 \mathrm{~Hz}, 3 \mathrm{H}), 0.69(\mathrm{dd}, J=6.7,1.6 \mathrm{~Hz}, 3 \mathrm{H}) .{ }^{13} \mathbf{C} \mathbf{N M R}\left(101 \mathrm{MHz}, \mathrm{CDCl}_{3}\right): \delta$ (ppm) $157.7(\mathrm{~d}, J=1.8 \mathrm{~Hz}), 155.8,153.3,142.0,139.3,138.2(\mathrm{~d}, J=29.8 \mathrm{~Hz}), 135.4,131.3,131.11(\mathrm{~d}, J=$ $3.7 \mathrm{~Hz}), 129.38-128.62(\mathrm{~m}), 120.6(\mathrm{~d}, J=2.5 \mathrm{~Hz}), 117.9,105.8(\mathrm{~d}, J=4.4 \mathrm{~Hz}), 72.5(\mathrm{~d}, J=7.0 \mathrm{~Hz}), 56.1$, 55.6, 40.3, 27.7, 24.7, 23.6 (d, $J=3.5 \mathrm{~Hz}), 23.0,22.6$. HRMS (EI): calcd for $\mathrm{C}_{22} \mathrm{H}_{26} \mathrm{FNO}_{2}{ }^{+}[\mathrm{M}]^{+}: 355.1948$, found 355.1948. IR: $v\left(\mathrm{~cm}^{-1}\right) 3212$ (br. w), 3076 (w), 2955 (m), 2932 (w), 2867 (w), 2833 (w), 1622 
(m), 1509 (s), 1465 (m), 1427 (m), 1352 (m), 1309 (m), 1267 (m), 1229 (vs), 119 (m), 1175 (w), 1137 (m), 1085 (m), 1028 (m), 1000 (m), 907 (s), 830 (s), 795 (m), 732 (vs).

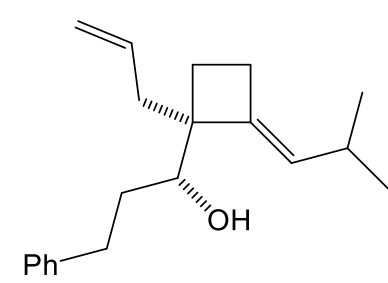

$\left(R^{*}\right)-1-\left(\left(R^{*}, E\right)-1\right.$-allyl-2-(2-methylpropylidene)cyclobutyl)-3-phenylpropan-1-ol $(\mathbf{4 k})$

Using allylzinc bromide as the zinc organyl, isopropylmagnesium chloride as the Grignard species, 2(dichloromethyl)-5,5-dimethyl-1,3,2-dioxaborinane as the boronic ester and 3-phenylpropanal according to general procedure A, provided $\mathbf{4 k}(72 \mathrm{mg}, 51 \%)$ as a colourless oil in 99:1 $d r$.

Colourless oil, $\boldsymbol{R}_{\mathbf{f}}=0.13$ (50:1 hexane:EtOAc, UV, PAA). ${ }^{1} \mathbf{H}$ NMR $\left(400 \mathrm{MHz}, \mathrm{CDCl}_{3}\right): \delta(\mathrm{ppm}) 7.37-7.12$ $(\mathrm{m}, 5 \mathrm{H}), 6.00-5.75(\mathrm{~m}, 1 \mathrm{H}), 5.10-5.04(\mathrm{~m}, 2 \mathrm{H}), 5.02(\mathrm{~s}, 1 \mathrm{H}), 3.55(\mathrm{~d}, J=10.2 \mathrm{~Hz}, 1 \mathrm{H}), 3.01-2.90(\mathrm{~m}$, 1H), $2.69-2.59(\mathrm{~m}, 1 \mathrm{H}), 2.51-2.35(\mathrm{~m}, 3 \mathrm{H}), 2.34-2.23(\mathrm{~m}, 1 \mathrm{H}), 2.16-2.08(\mathrm{~m}, 1 \mathrm{H}), 1.90-1.78(\mathrm{~m}$, 2H), $1.78-1.65(\mathrm{~m}, 3 \mathrm{H}), 0.95(\mathrm{~d}, J=6.7 \mathrm{~Hz}, 6 \mathrm{H}) .{ }^{13} \mathrm{C} \mathbf{N M R}\left(101 \mathrm{MHz}, \mathrm{CDCl}_{3}\right): \delta(\mathrm{ppm})$ 142.6, 141.5, 135.9, 130.1, 128.6, 128.5, 125.9, 117.1, 75.8, 54.2, 37.8, 33.5, 33.3, 27.8, 24.5, 23.6, 23.3, 23.1. HRMS (EI): calcd for $\mathrm{C}_{20} \mathrm{H}_{26}{ }^{+}\left[\mathrm{M}-\mathrm{H}_{2} \mathrm{O}\right]^{+}:$266.2035, found 266.2023. IR: $v$ ( $\left.\mathrm{cm}^{-1}\right) 3466$ (br. vw), 3064 (vw), 3027 (w), 2953 (m), 2924 (m), 2866 (w), 1639 (w), 1604 (w), 1496 (w), 1454 (m), 1415 (w), 1381 (w), 1361 (w), $1304(\mathrm{w}), 1262$ (w), $1176(\mathrm{w}), 1056$ (m), 1030 (m), 999 (m), 910 (s), 853 (w), 741 (m), 735 (m), 698 (vs).

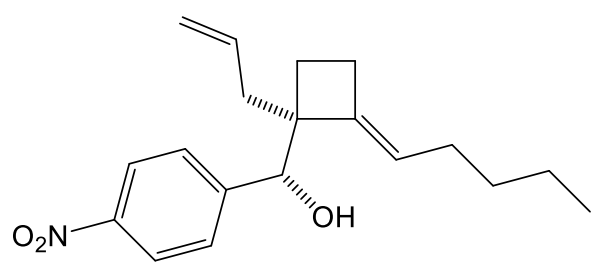

$\left(R^{*}\right)-\left(\left(R^{*}, E\right)-1-\right.$ allyl-2-pentylidenecyclobutyl $)(4-$ nitrophenyl)methanol (4I)

Using allylzinc bromide as the zinc organyl, butyllithium as the lithium organyl, 2-(dichloromethyl)-5,5dimethyl-1,3,2-dioxaborinane as the boronic ester and 4-nitrobenzaldehyde according to general procedure A, provided $4 \mathbf{l}$ (118 $\mathrm{mg}, 75 \%)$ as white crystals in 99:1 $d r$.

White crystals, $\mathrm{mp}=58-60{ }^{\circ} \mathrm{C}, \boldsymbol{R}_{\mathbf{f}}=0.24$ (4:1 hexane:EtOAc, UV, PAA). ${ }^{1} \mathbf{H}$ NMR $\left(400 \mathrm{MHz}, \mathrm{CDCl}_{3}\right): \delta$ (ppm) $8.25-8.05(\mathrm{~m}, 2 \mathrm{H}), 7.69-7.45(\mathrm{~m}, 2 \mathrm{H}), 5.91-5.79(\mathrm{~m}, 1 \mathrm{H}), 5.33-5.22(\mathrm{~m}, 1 \mathrm{H}), 5.15-4.94(\mathrm{~m}$, $2 \mathrm{H}), 4.87(\mathrm{~d}, J=3.2 \mathrm{~Hz}, 1 \mathrm{H}), 2.56-2.29(\mathrm{~m}, 3 \mathrm{H}), 2.26-2.12(\mathrm{~m}, 1 \mathrm{H}), 2.05-1.94(\mathrm{~m}, 2 \mathrm{H}), 1.94-1.77(\mathrm{~m}$, $3 \mathrm{H}), 1.40-1.19(\mathrm{~m}, 4 \mathrm{H}), 0.90(\mathrm{t}, J=7.1 \mathrm{~Hz}, 3 \mathrm{H}) .{ }^{13} \mathbf{C} \mathbf{N M R}\left(101 \mathrm{MHz}, \mathrm{CDCl}_{3}\right): \delta(\mathrm{ppm})$ 148.7, 147.3, 142.6, 135.2, 128.3, 124.2, 122.9, 117.9, 77.1, 54.9, 38.8, 31.8, 27.7, 24.7, 23.2, 22.5, 14.1. HRMS (EI): calcd for $\mathrm{C}_{19} \mathrm{H}_{23} \mathrm{NO}_{2}{ }^{+}\left[\mathrm{M}-\mathrm{H}_{2} \mathrm{O}\right]^{+}:$297.1729, found 297.1714. IR: $v\left(\mathrm{~cm}^{-1}\right) 3534$ (br. vw), 3076 (vw), 2955 (w), 2866 (w), 2856 (w), 1637 (w), 1604 (w), 1518 (s), 1457 (w), 1437 (w), 1418 (w), 1345 (vs), 1316 (w), $1286(w), 1245$ (w), 1193 (w), 1109 (w), 1041 (w), $1014(w), 999$ (w), $914(\mathrm{~m}), 856(\mathrm{~m}), 814(\mathrm{w}), 741(\mathrm{w})$, $706(\mathrm{~m})$. 


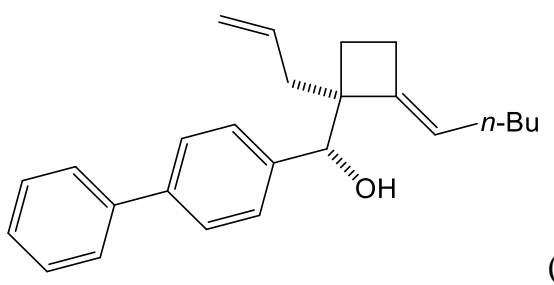

$$
\begin{gathered}
\left(R^{*}\right) \text {-[1,1'-biphenyl]-4-yl }\left(\left(R^{*}, E\right)\right. \text {-1-allyl-2- } \\
\text { pentylidenecyclobutyl)methanol (4m) }
\end{gathered}
$$

Using allylzinc bromide as the zinc organyl, $n$-butyllithium as the lithium species, 2-(dichloromethyl)-5,5dimethyl-1,3,2-dioxaborinane as the boronic ester and 4-biphenylcarbaldehyde according to general procedure A, provided $\mathbf{4 m}(128 \mathrm{mg}, 75 \%)$ as a colourless oil in 99:1 $d r$.

Colourless oil, $\boldsymbol{R}_{\mathbf{f}}=0.21$ (10:1 hexane:EtOAc, UV, PAA). ${ }^{1} \mathbf{H}$ NMR (400 MHz, $\left.\mathrm{CDCl}_{3}\right): 7.64-7.59$ (m, 2H), $7.58-7.53(\mathrm{~m}, 2 \mathrm{H}), 7.49-7.42(\mathrm{~m}, 4 \mathrm{H}), 7.38-7.32(\mathrm{~m}, 1 \mathrm{H}), 5.90(\mathrm{~m}, 1 \mathrm{H}), 5.31$ (ddt, J = 7.3, 5.3, $2.7 \mathrm{~Hz}$, $1 \mathrm{H}), 5.10-4.98(\mathrm{~m}, 2 \mathrm{H}), 4.82(\mathrm{~d}, \mathrm{~J}=3.0 \mathrm{~Hz}, 1 \mathrm{H}), 2.51-2.41(\mathrm{~m}, 1 \mathrm{H}), 2.39-2.31(\mathrm{~m}, 2 \mathrm{H}), 2.27(\mathrm{~d}, \mathrm{~J}=3.3$ $\mathrm{Hz}, 1 \mathrm{H}), 2.15-1.99(\mathrm{~m}, 2 \mathrm{H}), 1.96-1.88(\mathrm{~m}, 2 \mathrm{H}), 1.85$ (ddd, J = 11.7, 9.7, 7.6 Hz, 1H), $1.34(\mathrm{~m}, 4 \mathrm{H}), 0.91$ $(\mathrm{t}, \mathrm{J}=7.1 \mathrm{~Hz}, 3 \mathrm{H}) .{ }^{13} \mathrm{C}$ NMR $\left(101 \mathrm{MHz}, \mathrm{CDCl}_{3}\right)$ : 143.2, 141.0, 140.2, 136.0, 128.9, 128.0, 127.3, 127.3, 127.2, 126.4, 123.6, 117.3, 77.9, 55.0, 38.4, 31.9, 27.7, 24.7, 23.4, 22.5, 14.2. LRMS (EI): 346 (8), 331 (12), 281 (35), 253 (19), 207 (100), 183 (64). HRMS (EI): calcd for $\mathrm{C}_{25} \mathrm{H}_{30} \mathrm{O}^{+}[\mathrm{M}]^{+}:$346.2297, found 346.2283.

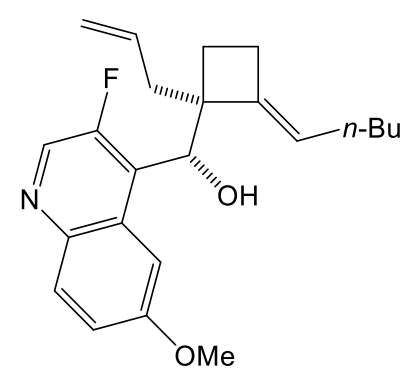

$\left(S^{*}\right)-\left(\left(R^{*}, E\right)-1\right.$-allyl-2-pentylidenecyclobutyl)(3-fluoro-6-methoxyquinolin-4yl)methanol (4n)

Using allylzinc bromide as the zinc organyl, $n$-butyllithium as the lithium species, 2-(dichloromethyl)-5,5dimethyl-1,3,2-dioxaborinane as the boronic ester and 3-fluoro-6-methoxyquinoline-4-carbaldehyde according to general procedure A, provided $4 \mathbf{n}(110 \mathrm{mg}, 60 \%)$ as a slightly yellow oil in 99:1 $d r$.

Colourless oil, $\boldsymbol{R}_{\mathbf{f}}=0.21$ (10:1 hexane:EtOAc, UV, PAA). ${ }^{1} \mathbf{H}$ NMR $\left(400 \mathrm{MHz}, \mathrm{CDCl}_{3}\right): \delta(\mathrm{ppm}){ }^{\mathbf{1 3}} \mathbf{C} \mathbf{~ N M R}$ (101 MHz, $\left.\mathrm{CDCl}_{3}\right): 157.8,154.6(\mathrm{~d}, J=252.4 \mathrm{~Hz}), 142.2,141.7,138.4(\mathrm{~d}, J=31.8 \mathrm{~Hz}), 135.5,131.3,129.2$ $(\mathrm{d}, J=2.5 \mathrm{~Hz}), 128.5(\mathrm{~d}, J=7.7 \mathrm{~Hz}), 124.2,124.2,120.7,118.0,105.7(\mathrm{~d}, J=4.9 \mathrm{~Hz}), 72.6,56.4,55.6,39.9$, 31.7, 27.6, 24.7, 23.6, 22.4, 14.1. LRMS (EI): 369 (90), 351 (21), 281 (100). HRMS (EI): calcd for $\mathrm{C}_{23} \mathrm{H}_{28} \mathrm{FNO}_{2}^{+}[\mathrm{M}]^{+}:$369.2104, found 369.2097. 


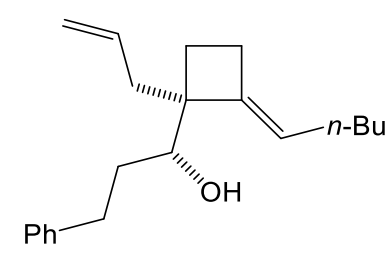

$\left(R^{*}\right)-1-\left(\left(R^{*}, E\right)-1\right.$-allyl-2-pentylidenecyclobutyl)-3-phenylpropan-1-ol (4o)

Using allylzinc bromide as the zinc organyl, $n$-butyllithium as the lithium species, 2-(dichloromethyl)-5,5dimethyl-1,3,2-dioxaborinane as the boronic ester and hydrocinnamaldehyde according to general procedure A, provided 4o (101 mg, 68\%) as a colourless oil in 99:1 $d r$.

Colourless oil, $\boldsymbol{R}_{\mathbf{f}}=0.42\left(10: 1\right.$ hexane:EtOAc, UV, PAA). ${ }^{1} \mathbf{H}$ NMR $\left(400 \mathrm{MHz}, \mathrm{CDCl}_{3}\right): \delta(\mathrm{ppm}){ }^{\mathbf{1 3}} \mathbf{C} \mathbf{~ N M R}$ (101 MHz, $\left.\mathrm{CDCl}_{3}\right): \delta 143.7,142.6,136.0,128.7,128.6,128.5,128.4,126.4,125.9,122.8,117.0,75.7,54.4$, 37.9, 33.5, 33.4, 31.9, 27.6, 24.5, 23.6, 22.4, 14.1. LRMS (EI): 298 (3), 280 (10), 257 (11), 239 (21), 164 (100). HRMS (EI): calcd for $\mathrm{C}_{21} \mathrm{H}_{30} \mathrm{O}^{+}[\mathrm{M}]^{+}:$298.2297, found 298.2302.

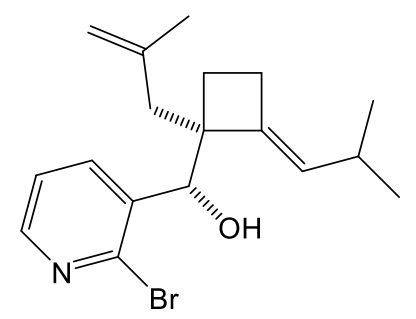

$\left(S^{*}\right)$-(2-bromopyridin-3-yl)((R*,E)-1-(2-methylallyl)-2-(2-methylpropylidene)cyclobutyl)methanol (4p)

Using (2-methylallyl)zinc bromide as the zinc organyl, isopropylmagnesium chloride as the Grignard species, 2-(dichloromethyl)-5,5-dimethyl-1,3,2-dioxaborinane as the boronic ester and 2-bromo-3pyridinecarboxaldehyde according to general procedure $\mathbf{A}$, provided $\mathbf{4 p}$ (142 $\mathrm{mg}, 81 \%)$ as a colourless oil in 99:1 $d r$.

Colourless oil, $\boldsymbol{R}_{\mathbf{f}}=0.15$ (10:1 hexane:EtOAc, UV, PAA). ${ }^{1} \mathbf{H}$ NMR (400 MHz, $\left.\mathrm{CDCl}_{3}\right): \delta(\mathrm{ppm}) 8.23(\mathrm{dd}, J$ = 4.6, $2.0 \mathrm{~Hz}, 1 \mathrm{H}), 7.99(\mathrm{dd}, J=7.7,2.0 \mathrm{~Hz}, 1 \mathrm{H}), 7.25(\mathrm{dd}, J=7.7,4.6 \mathrm{~Hz}, 1 \mathrm{H}), 5.29-5.16(\mathrm{~m}, 2 \mathrm{H}), 4.86$ (dt, $J=2.9,1.5 \mathrm{~Hz}, 1 \mathrm{H}), 4.79(\mathrm{~s}, 1 \mathrm{H}), 2.66(\mathrm{~d}, J=3.8 \mathrm{~Hz}, 1 \mathrm{H}), 2.44-2.26(\mathrm{~m}, 4 \mathrm{H}), 2.23-1.84(\mathrm{~m}, 3 \mathrm{H})$, $1.79(\mathrm{~s}, 3 \mathrm{H}), 0.87(\mathrm{~d}, J=6.7 \mathrm{~Hz}, 3 \mathrm{H}), 0.81(\mathrm{~d}, J=6.7 \mathrm{~Hz}, 3 \mathrm{H}) .{ }^{13} \mathbf{C} \mathbf{N M R}\left(101 \mathrm{MHz}, \mathrm{CDCl}_{3}\right): \delta(\mathrm{ppm}) 148.9$, 144.8, 143.6, 139.9, 138.9, 137.7, 131.2, 122.5, 114.4, 75.2, 55.6, 44.9, 27.7, 25.7, 24.9, 23.3, 22.9, 22.6. HRMS (EI): calcd for $\mathrm{C}_{18} \mathrm{H}_{24} \mathrm{BrNO}^{+}[\mathrm{M}]^{+}: 349.1041$, found 349.1048. IR: $v$ (cm $\left.{ }^{-1}\right) 3325$ (br. w), 3072 (w), 2954 (m), 2921 (m), 2867 (w), 1642 (w), 1576 (m), 1561 (m), 1463 (m), 1445 (m9, 1403 (s), 1374 (m), 1366 (m), $1260(\mathrm{w}), 1181(\mathrm{w}), 1117(\mathrm{~m}), 1077(\mathrm{~m}), 1055(\mathrm{~m}), 1027$ (s), 978 (w), $948(\mathrm{w}), 908$ (m), $890(\mathrm{~s}), 857$ (m), $836(\mathrm{w}), 806(\mathrm{~m}), 780(\mathrm{~m}), 732$ (vs), 702 (s). 


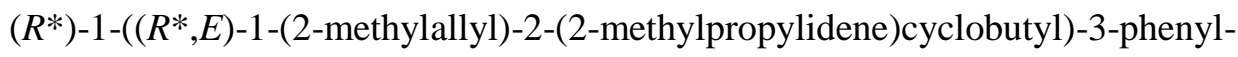
propan-1-ol (4q)

Using (2-methylallyl)zinc bromide as the zinc organyl, isopropylmagnesium chloride as the Grignard species, 2-(dichloromethyl)-5,5-dimethyl-1,3,2-dioxaborinane as the boronic ester and 3-phenylpropanal according to general procedure $\mathbf{A}$, provided $\mathbf{4 q}(111 \mathrm{mg}, 74 \%)$ as a colourless oil in 99:1 $d r$.

Colourless oil, $\boldsymbol{R}_{\mathbf{f}}=0.10$ (50:1 hexane:EtOAc, UV, PAA). ${ }^{1} \mathbf{H}$ NMR (400 MHz, $\left.\mathrm{CDCl}_{3}\right): \delta(\mathrm{ppm}) 7.43-7.04$ (m, 5H), $5.13-5.05(\mathrm{~m}, 1 \mathrm{H}), 4.83-4.79(\mathrm{~m}, 1 \mathrm{H}), 4.72(\mathrm{~s}, 1 \mathrm{H}), 3.58-3.50(\mathrm{~m}, 1 \mathrm{H}), 3.02-2.90(\mathrm{~m}, 1 \mathrm{H})$, $2.70-2.58(\mathrm{~m}, 1 \mathrm{H}), 2.52-2.43(\mathrm{~m}, 2 \mathrm{H}), 2.41-2.25(\mathrm{~m}, 2 \mathrm{H}), 2.27-2.12(\mathrm{~m}, 1 \mathrm{H}), 2.02-1.80(\mathrm{~m}, 4 \mathrm{H})$, $1.77(\mathrm{~s}, 3 \mathrm{H}), 1.75-1.64(\mathrm{~m}, 1 \mathrm{H}), 0.95(\mathrm{dd}, J=6.7,4.9 \mathrm{~Hz}, 6 \mathrm{H}) .{ }^{13} \mathbf{C} \mathbf{N M R}\left(101 \mathrm{MHz}, \mathrm{CDCl}_{3}\right): \delta(\mathrm{ppm})$ 144.6, 142.6, 142.4, 129.9, 128.6, 128.5, 125.9, 113.9, 76.3, 54.3, 42.3, 33.6, 33.5, 27.8, 25.2, 24.7, 23.3, 22.8. HRMS (EI): calcd for $\mathrm{C}_{21} \mathrm{H}_{30} \mathrm{O}^{+}[\mathrm{M}]^{+}:$298.2297, found 298.2290. IR: $v\left(\mathrm{~cm}^{-1}\right) 3447$ (br. vw), 3065 (vw), 3027 (w), 2953 (m), 2925 (m), 2866 (w), 1642 (w), 1454 (m), 1378 (w), 1362 (m), 1287 (w), 1256 (w), $1174(\mathrm{w}), 1040$ (m), $929(\mathrm{~m}), 889(\mathrm{~m}), 842$ (w), 747 (m), 734 (m), 698 (vs).

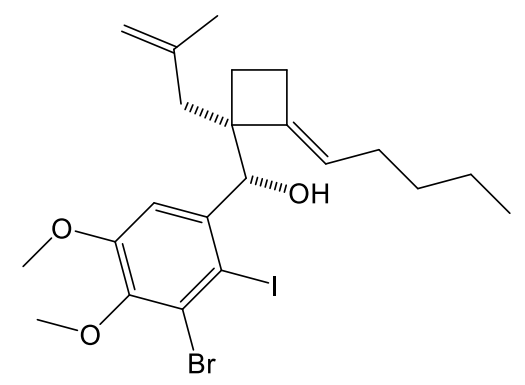

$\left(S^{*}\right)$-(3-bromo-2-iodo-4,5-dimethoxyphenyl) $\left(\left(R^{*}, E\right)\right.$-1-(2-methylallyl)-2pentylidenecyclobutyl)methanol (4r)

Using (2-methylallyl)zinc bromide as the zinc organyl, butyllithium as the lithium organyl, 2(dichloromethyl)-5,5-dimethyl-1,3,2-dioxaborinane as the boronic ester and 3-bromo-2-iodo-4,5dimethoxybenzaldehyde according to general procedure $\mathbf{A}$, provided $\mathbf{4 r}(135 \mathrm{mg}, 50 \%)$ as a colourless oil in 99:1 $d r$.

Colourless crystals, $\mathrm{mp}=81-83^{\circ} \mathrm{C}, \boldsymbol{R}_{\mathbf{f}}=0.07$ (20:1 hexane:EtOAc, UV, PAA). ${ }^{1} \mathbf{H}$ NMR $(400 \mathrm{MHz}$, $\left.\mathrm{CDCl}_{3}\right): \delta(\mathrm{ppm}) 7.32(\mathrm{~s}, 1 \mathrm{H}), 5.48-5.40(\mathrm{~m}, 1 \mathrm{H}), 5.27(\mathrm{~d}, J=3.8 \mathrm{~Hz}, 1 \mathrm{H}), 4.89-4.83(\mathrm{~m}, 1 \mathrm{H}), 4.78(\mathrm{~s}$, $1 \mathrm{H}), 3.88(\mathrm{~s}, 3 \mathrm{H}), 3.83(\mathrm{~s}, 3 \mathrm{H}), 2.49-2.27(\mathrm{~m}, 5 \mathrm{H}), 2.26-2.12(\mathrm{~m}, 1 \mathrm{H}), 2.10-1.94(\mathrm{~m}, 1 \mathrm{H}), 1.89-1.71$ $(\mathrm{m}, 2 \mathrm{H}), 1.79(\mathrm{~s}, 3 \mathrm{H}), 1.36-1.09(\mathrm{~m}, 4 \mathrm{H}), 0.84(\mathrm{t}, J=6.9 \mathrm{~Hz}, 3 \mathrm{H}) .{ }^{13} \mathbf{C} \mathbf{N M R}\left(101 \mathrm{MHz}, \mathrm{CDCl}_{3}\right): \delta(\mathrm{ppm})$ 153.2, 146.5, 144.8, 143.0, 142.8, 126.3, 123.9, 114.5, 111.4, 97.9, 82.6, 60.5, 56.5, 56.2, 45.0, 31.7, 27.6, 26.1, 24.9, 24.3, 22.5, 14.2. HRMS (EI): calcd for $\mathrm{C}_{22} \mathrm{H}_{30} \mathrm{BrIO}_{3}{ }^{+}[\mathrm{M}]^{+}:$548.0423, found 548.0420. IR: $v\left(\mathrm{~cm}^{-}\right.$ 1) 3467 (br. m), 3068 (vw), 2996 (w), 2953 (m), 2928 (m), 2870 (w), 2849 (w), 1643 (w), 1581 (w), 1462 (s), 1444 (m), 1419 8s), 1375 (m), 1344 (m), 1277 (s), 1248 (m), 1193 (m), 1158 (m), 1132 (m), 1066 (m), 1052 (s), 1032 (s), 996 (vs), 969 (m), 912 (w), 889 (s), 866 (s), 814 (m), 791 (m), 741 (m), 715 (w), 664 (m). 


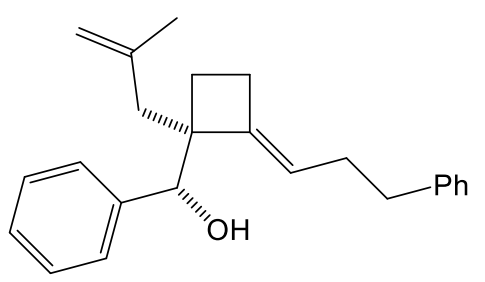

$\left(R^{*}\right)-\left(\left(R^{*}, E\right)-1-(2-m e t h y l a l l y l)-2-(3-\right.$

phenylpropylidene)cyclobutyl)(phenyl)-methanol (4s)

Using (2-methylallyl)zinc bromide as the zinc organyl, phenethylmagnesium bromide as the Grignard species, 2-(dichloromethyl)-5,5-dimethyl-1,3,2-dioxaborinane as the boronic ester and benzaldehyde according to general procedure $\mathbf{A}$, provided $\mathbf{4 s}(135 \mathrm{mg}, 81 \%)$ as a colourless oil in 99:1 $d r$.

Colourless oil, $\boldsymbol{R}_{\mathbf{f}}=0.22$ (20:1 hexane:EtOAc, UV, PAA). , ${ }^{1} \mathbf{H}$ NMR $\left(400 \mathrm{MHz}, \mathrm{CDCl}_{3}\right): \delta(\mathrm{ppm}) 7.44-7.09$ (m, 10H), $5.29-5.20(\mathrm{~m}, 1 \mathrm{H}), 4.83(\mathrm{~s}, 1 \mathrm{H}), 4.71(\mathrm{~d}, J=4.0 \mathrm{~Hz}, 1 \mathrm{H}), 4.69(\mathrm{~s}, 1 \mathrm{H}), 2.75-2.58(\mathrm{~m}, 2 \mathrm{H}), 2.35$ - 1.92 (m, 9H), 1.72 (s, 3H). ${ }^{13}$ C NMR (101 MHz, $\left.\mathrm{CDCl}_{3}\right)$ : $\delta$ (ppm) 145.2, 144.4, 142.1, 141.3, 128.5, 128.4, 127.6, 127.3, 125.9, 122.1, 113.8, 78.3, 54.9, 42.7, 35.7, 29.6, 25.4, 24.9, 24.4. HRMS (EI): calcd for $\mathrm{C}_{24} \mathrm{H}_{28} \mathrm{O}^{+}[\mathrm{M}]^{+}:$332.2140, found 332.2132. IR: $v\left(\mathrm{~cm}^{-1}\right) 3554$ (br. vw), 3463 (br. vw), 3080 (vw), 3063 (vw), 3028 (w), 2917 (w), 2853 (w), 1641 (w), 1603 (w), 1495 (w), 1452 (m), 1417 (w), 1374 (w), 1252 (w), 1194 (w), $1081(\mathrm{w}), 1036(\mathrm{~m}), 1022(\mathrm{~m}), 983(\mathrm{w}), 889(\mathrm{~m}), 847$ (w), $734(\mathrm{~m}), 698$ (vs).

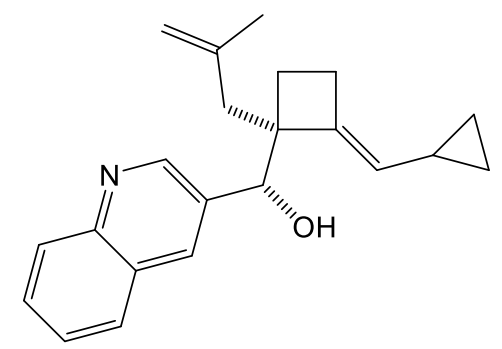

$\left(S^{*}\right)-\left(\left(R^{*}, E\right)-2-(\right.$ cyclopropylmethylene)-1-(2methylallyl)cyclobutyl)(quinolin-3-yl)-methanol (4t)

Using (2-methylallyl)zinc bromide as the zinc organyl, cyclopropylmagnesium bromide as the Grignard species, 2-(dichloromethyl)-5,5-dimethyl-1,3,2-dioxaborinane as the boronic ester and quinoline-3carboxaldehyde according to general procedure A, provided $\mathbf{4 t}(80 \mathrm{mg}, 50 \%)$ as a white solid in 99:1 $d r$.

White solid, $\mathrm{mp}=139-141{ }^{\circ} \mathrm{C}, \boldsymbol{R}_{\mathbf{f}}=0.12$ (5:1 hexane:EtOAc, UV, PAA). ${ }^{1} \mathbf{H}$ NMR $\left(400 \mathrm{MHz}, \mathrm{CDCl}_{3}\right): \delta$ (ppm) $8.84(\mathrm{~d}, J=2.2 \mathrm{~Hz}, 1 \mathrm{H}), 8.18(\mathrm{~d}, J=2.1 \mathrm{~Hz}, 1 \mathrm{H}), 8.06(\mathrm{~d}, J=8.5 \mathrm{~Hz}, 1 \mathrm{H}), 7.77(\mathrm{~d}, J=8.2 \mathrm{~Hz}, 1 \mathrm{H})$, 7.67 (ddd, $J=8.4,6.8,1.5 \mathrm{~Hz}, 1 \mathrm{H}), 7.52$ (ddd, $J=8.1,6.8,1.2 \mathrm{~Hz}, 1 \mathrm{H}), 4.97$ (s, 1H), 4.86 (s, 1H), 4.73 (s, $1 \mathrm{H}), 4.65-5.58(\mathrm{~m}, 1 \mathrm{H}), 3.20(\mathrm{~s}, 1 \mathrm{H}), 2.56-2.00(\mathrm{~m}, 6 \mathrm{H}), 1.76(\mathrm{~s}, 3 \mathrm{H}), 1.29-1.08(\mathrm{~m}, 1 \mathrm{H}), 0.75-0.56$ (m, 2H), $0.37-0.17(\mathrm{~m}, 2 \mathrm{H}) .{ }^{13} \mathbf{C}$ NMR (101 MHz, $\left.\mathrm{CDCl}_{3}\right): \delta$ (ppm) 150.6, 147.4, 144.3, 141.7, 134.7, 134.4, 129.3, 129.1, 128.0, 127.6, 127.5, 126.7, 114.3, 76.2, 54.9, 43.8, 25.9, 24.8, 24.8, 10.3, 6.5, 6.4. HRMS (EI): calcd for $\mathrm{C}_{22} \mathrm{H}_{25} \mathrm{NO}^{+}[\mathrm{M}]^{+}:$319.1936, found 319.1928. IR: $v\left(\mathrm{~cm}^{-1}\right) 3142$ (br. w), 3080 (w), $3061(w), 3001(w), 2954$ (w), 2920 (w), 2362 (w), 2335 (vw), 1640 (w), 1557 (w), 1498 (m), 1444 (w), 1420 (w), 1380 (m), 1316 (w), 1283 (w), 1229 (w), 1195 (w), 1148 (w), 1137 (w), 1124 (w), 1077 (w), 1046 (s), $1012(\mathrm{w}), 956(\mathrm{~m}), 905$ (m), 892 (s), 804 (m), 799 (w), 790 (s), 753 (vs). 


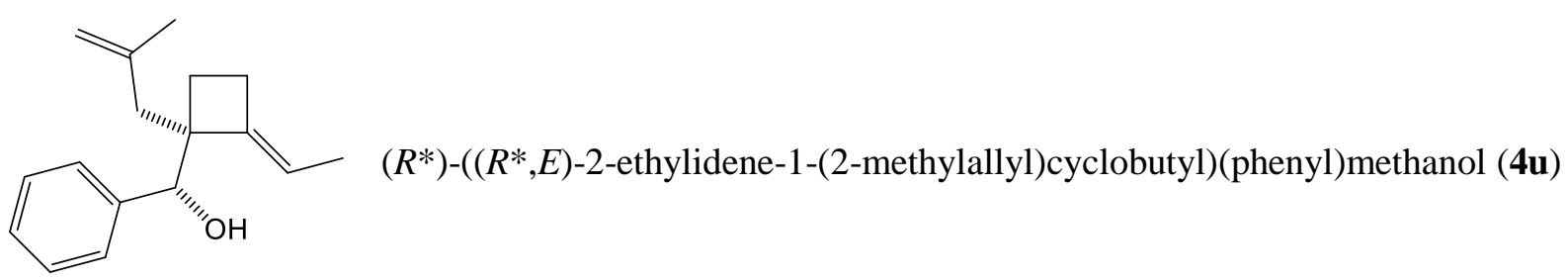

Using (2-methylallyl)zinc bromide as the zinc organyl, methylmagnesium chloride as the Grignard species, 2-(dichloromethyl)-5,5-dimethyl-1,3,2-dioxaborinane as the boronic ester and benzaldehyde according to general procedure A, provided $\mathbf{4 u}(109 \mathrm{mg}, 90 \%)$ as a colourless oil in 99:1 $d r$.

Using the same procedure, but adding benzaldehyde directly, without washing and without changing the solvent to $\mathrm{CH}_{2} \mathrm{Cl}_{2}$ afforded, after $24 \mathrm{~h}$ reaction time, $4 \mathbf{u}(101 \mathrm{mg}, 83 \%)$ in 99:1 $d r$.

Colourless oil, $\boldsymbol{R}_{\mathbf{f}}=0.16$ (50:1 hexane:EtOAc, UV, PAA). ${ }^{1} \mathbf{H}$ NMR $\left(400 \mathrm{MHz}, \mathrm{CDCl}_{3}\right): \delta(\mathrm{ppm}) 7.43-7.34$ (m, 2H), $7.34-7.19$ (m, 3H), 5.29 (qt, $J=6.7,2.6 \mathrm{~Hz}, 1 \mathrm{H}), 4.85(\mathrm{~s}, 1 \mathrm{H}), 4.79$ (d, $J=3.7 \mathrm{~Hz}, 1 \mathrm{H}), 4.70$ (s, $1 \mathrm{H}), 2.43-2.29(\mathrm{~m}, 3 \mathrm{H}), 2.29-2.06(\mathrm{~m}, 3 \mathrm{H}), 2.06-1.94(\mathrm{~m}, 1 \mathrm{H}), 1.74(\mathrm{~s}, 3 \mathrm{H}), 1.51$ (d, J=6.7 Hz, 3H). ${ }^{13}$ C NMR (101 MHz, $\left.\mathrm{CDCl}_{3}\right): \delta$ (ppm) 144.9, 144.6, 141.4, 127.6, 127.3, 117.4, 113.8, 78.4, 54.9, 42.6, 25.2, 24.9, 24.4, 13.1. HRMS (EI): calcd for $\mathrm{C}_{17} \mathrm{H}_{21} \mathrm{O}^{+}[\mathrm{M}-\mathrm{H}]^{+}$: 241.1592, found 241.1587. IR: $v\left(\mathrm{~cm}^{-1}\right) 3454$ (br. vw), 3068 (vw), 3031 (vw), 2966 (w), 2914 (w), 2856 (w), 1641 (w), 1494 (w), 1451 (m), 1421 (w), 1374 (w), 1193 (w), 1036 (m), 1022 (m), 973 (w), 908 (m), 890 (s), 838 (w), 768 (w), 733 (s), 701 (vs), $668(\mathrm{~m})$.

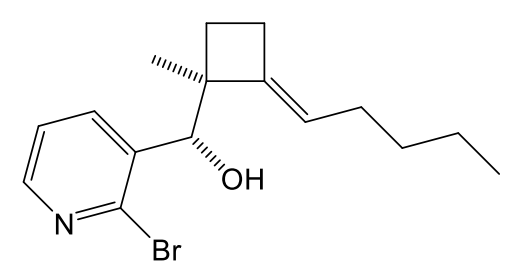

$\left(S^{*}\right)$-(2-bromopyridin-3-yl) $\left(\left(S^{*}, E\right)\right.$-1-methyl-2pentylidenecyclobutyl)methanol (4v)

Using butyllithium as the lithium organyl, 2-(dichloromethyl)-5,5-dimethyl-1,3,2-dioxaborinane as the boronic ester and 2-bromo-3-pyridinecarboxaldehyde according to general procedure $\mathbf{B}$, provided $\mathbf{4 v}$ (81 $\mathrm{mg}$, $50 \%$ ) as a colourless oil in 97:3 $d r$.

Colourless oil, $\boldsymbol{R}_{\mathbf{f}}=0.14$ (10:1 hexane:EtOAc, UV, PAA). ${ }^{1} \mathbf{H}$ NMR $\left(400 \mathrm{MHz}, \mathrm{CDCl}_{3}\right): \delta(\mathrm{ppm}) 8.27(\mathrm{dd}, J$ $=4.6,2.0 \mathrm{~Hz}, 1 \mathrm{H}), 7.90(\mathrm{dd}, J=7.7,2.0 \mathrm{~Hz}, 1 \mathrm{H}), 7.27(\mathrm{~d}, J=7.7,4.6 \mathrm{~Hz}, 1 \mathrm{H}), 5.26-5.16(\mathrm{~m}, 2 \mathrm{H}), 2.72-$ $2.46(\mathrm{~m}, 2 \mathrm{H}), 2.42-2.30(\mathrm{~m}, 2 \mathrm{H}), 1.97-1.87(\mathrm{~m}, 2 \mathrm{H}), 1.71-1.56(\mathrm{~m}, 1 \mathrm{H}), 1.40-1.22(\mathrm{~m}, 4 \mathrm{H}), 1.13(\mathrm{~s}$, 3H), $0.89(\mathrm{t}, J=7.1 \mathrm{~Hz}, 3 \mathrm{H}) .{ }^{13} \mathbf{C}$ NMR (101 MHz, $\left.\mathrm{CDCl}_{3}\right): \delta(\mathrm{ppm}) 149.1,145.2,143.2,138.4,138.2$, 122.7, 122.5, 74.9, 52.5, 31.9, 27.7, 27.6, 25.3, 22.4, 21.1, 14.2. HRMS (EI): calcd for $\mathrm{C}_{16} \mathrm{H}_{22} \mathrm{BrNO}^{+}[\mathrm{M}]^{+}$: 323.0885, found 323.0868. IR: $v\left(\mathrm{~cm}^{-1}\right) 3338$ (br. vw), 2957 (w), 2925 (w), 2871 (w), 2859 (w), 2241 (vw), 1576 (w), 1560 (w), 1455 (w); 1404 (m), 1373 (w), 1326 (w), 1260 (w), 1180 (w), 1116 (w), $1071($ w), 1056 (w), $1032(\mathrm{~m}), 906(\mathrm{~s}), 806(\mathrm{w}), 729(\mathrm{vs})$. 


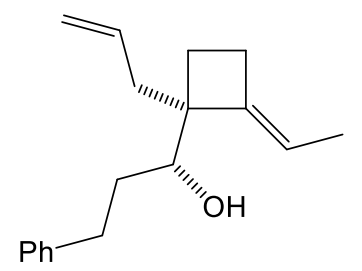

$\left(R^{*}\right)-1-\left(\left(R^{*}, E\right)-1-a l l y l-2-e t h y l i d e n e c y c l o b u t y l\right)-3-p h e n y l p r o p a n-1-o l ~(4 \mathbf{w})$

Using methylmagnesium chloride as the Grignard reagent, 2-(dichloromethyl)-5,5-dimethyl-1,3,2dioxaborinane as the boronic ester and hydrocinnamaldehyde according to general procedure $\mathbf{A}$, provided $\mathbf{4 w}$ (70 mg, 55\%) as a colourless oil in 99:1 $d r$.

Colourless oil, $\boldsymbol{R}_{\mathbf{f}}=0.40$ (10:1 hexane:EtOAc, UV, PAA). ${ }^{1} \mathbf{H}$ NMR (400 MHz, $\left.\mathrm{CDCl}_{3}\right): 7.25-7.19(\mathrm{~m}, 2 \mathrm{H})$, $7.17-7.08(\mathrm{~m}, 4 \mathrm{H}), 5.78(\mathrm{~m}, 1 \mathrm{H}), 5.20(\mathrm{qt}, \mathrm{J}=6.6,2.7 \mathrm{~Hz}, 1 \mathrm{H}), 5.00-4.96(\mathrm{~m}, 1 \mathrm{H}), 4.95-4.93(\mathrm{~m}, 1 \mathrm{H})$, $3.47(\mathrm{dd}, \mathrm{J}=10.3,2.0 \mathrm{~Hz}, 1 \mathrm{H}), 2.88(\mathrm{ddd}, \mathrm{J}=13.7,10.1,4.9 \mathrm{~Hz}, 1 \mathrm{H}), 2.55$ (ddd, J = 13.7, 9.6, $6.9 \mathrm{~Hz}, 1 \mathrm{H}$ ), $2.41-2.25(\mathrm{~m}, 3 \mathrm{H}), 2.14-1.99(\mathrm{~m}, 1 \mathrm{H}), 1.84-1.52(\mathrm{~m}, 4 \mathrm{H}), 1.44(\mathrm{dt}, \mathrm{J}=6.7,1.7 \mathrm{~Hz}, 3 \mathrm{H}) .{ }^{13} \mathbf{C} \mathbf{N M R}(101$ $\left.\mathrm{MHz}, \mathrm{CDCl}_{3}\right): 144.5,142.5,136.0,128.6,128.5,125.9,117.0,116.8,75.7,54.4,37.9,33.5,33.3,24.2$, 23.7, 13.2.HRMS (EI): calcd for $\mathrm{C}_{18} \mathrm{H}_{22}\left[\mathrm{M}-\mathrm{H}_{2} \mathrm{O}\right]: 238.1722$, found 238.1731 . 


\section{NMR Spectra:}

$\left(R^{*}\right)-\left(\left(R^{*}, E\right)-1-\right.$ allyl-2-ethylidenecyclobutyl)(phenyl)methanol (4a)

${ }^{1} \mathrm{H}$ NMR, $400 \mathrm{MHz}, \mathrm{CDCl}_{3}$

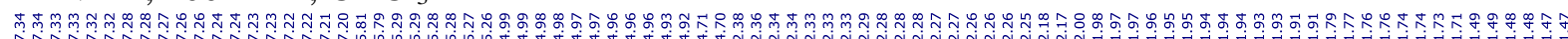
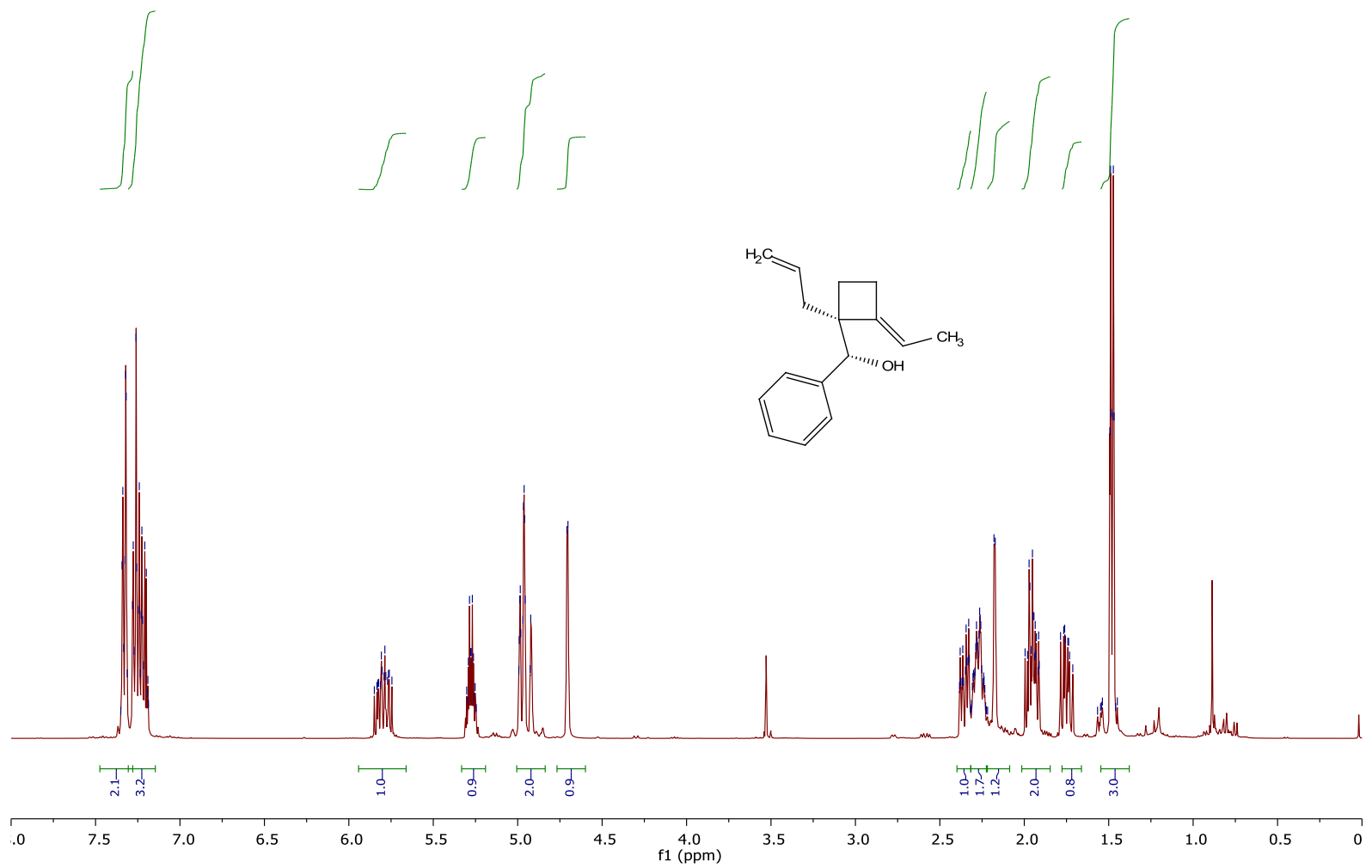

${ }^{13} \mathrm{C}$ NMR, $101 \mathrm{MHz}, \mathrm{CDCl}_{3}$

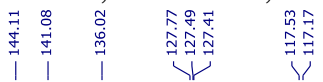

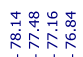

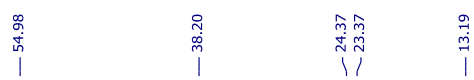
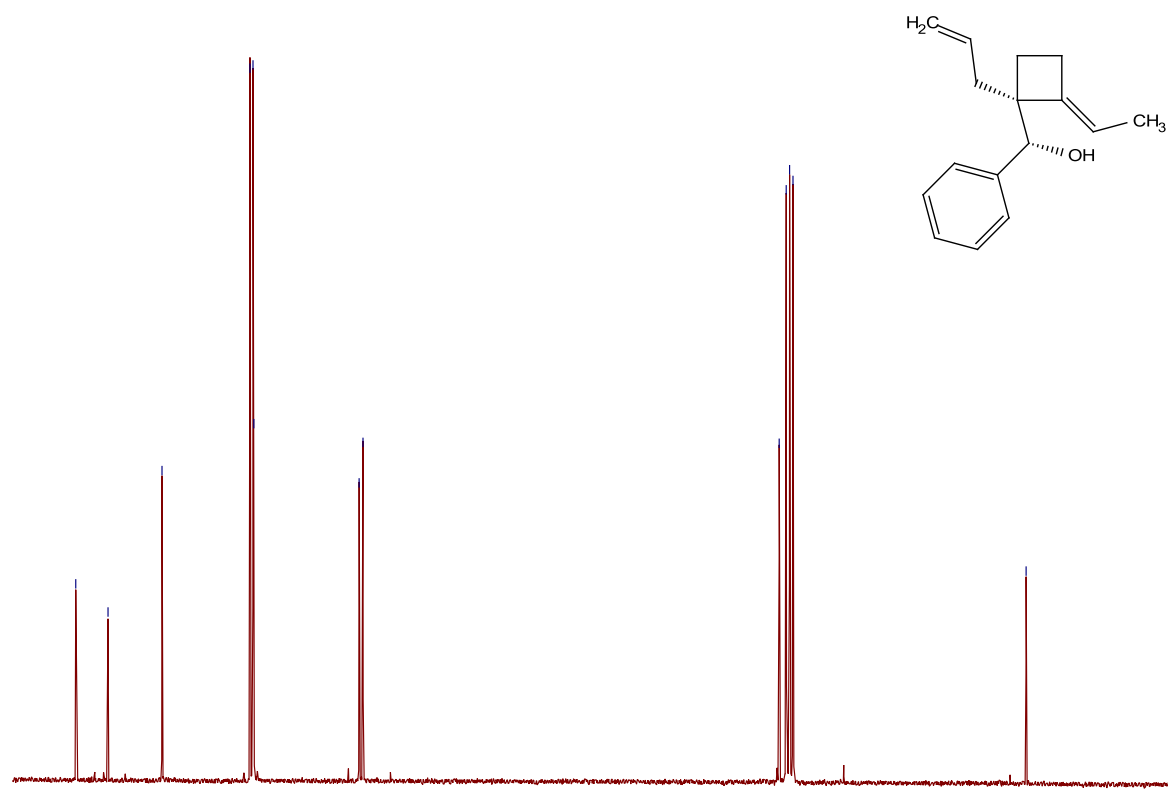

$\begin{array}{llllllllllllllll}50 & 145 & 140 & 135 & 130 & 125 & 120 & 115 & 110 & 105 & 100 & 95 & 90 & 85 & 80 & 75 \\ \mathrm{f} 1(\mathrm{ppm})\end{array}$ 
$\left(R^{*}\right)-\left(\left(R^{*}, E\right)-1-a l l y l-2-p r o p y l i d e n e c y c l o b u t y l\right)($ phenyl)methanol (4b)

${ }^{1} \mathrm{H}$ NMR, $400 \mathrm{MHz}, \mathrm{CDCl}_{3}$

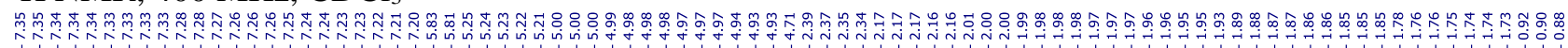
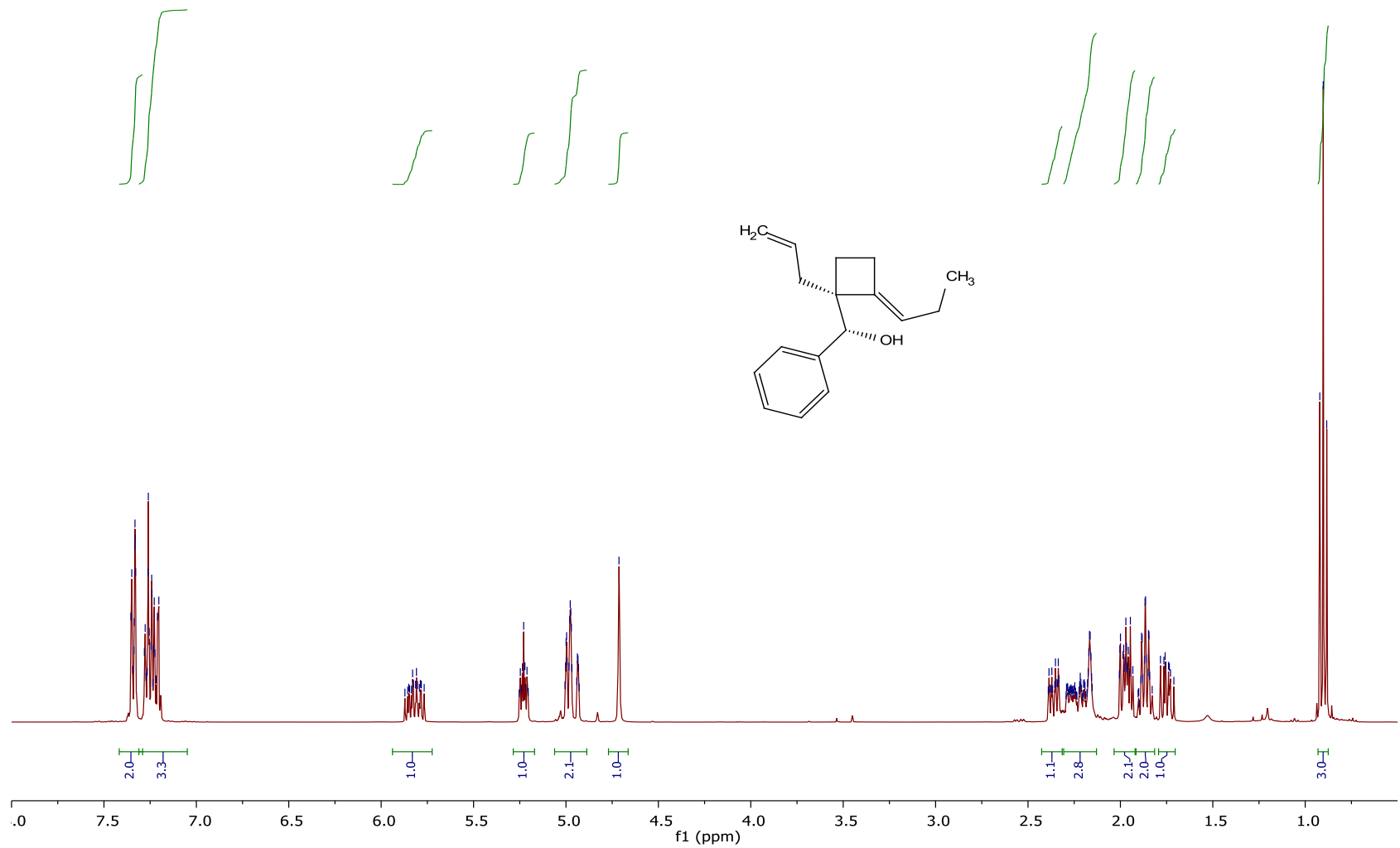

${ }^{13} \mathrm{C} \mathrm{NMR}, 101 \mathrm{MHz}, \mathrm{CDCl}_{3}$

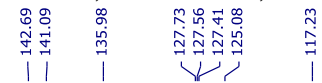

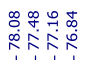

|

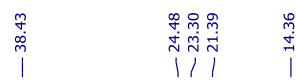

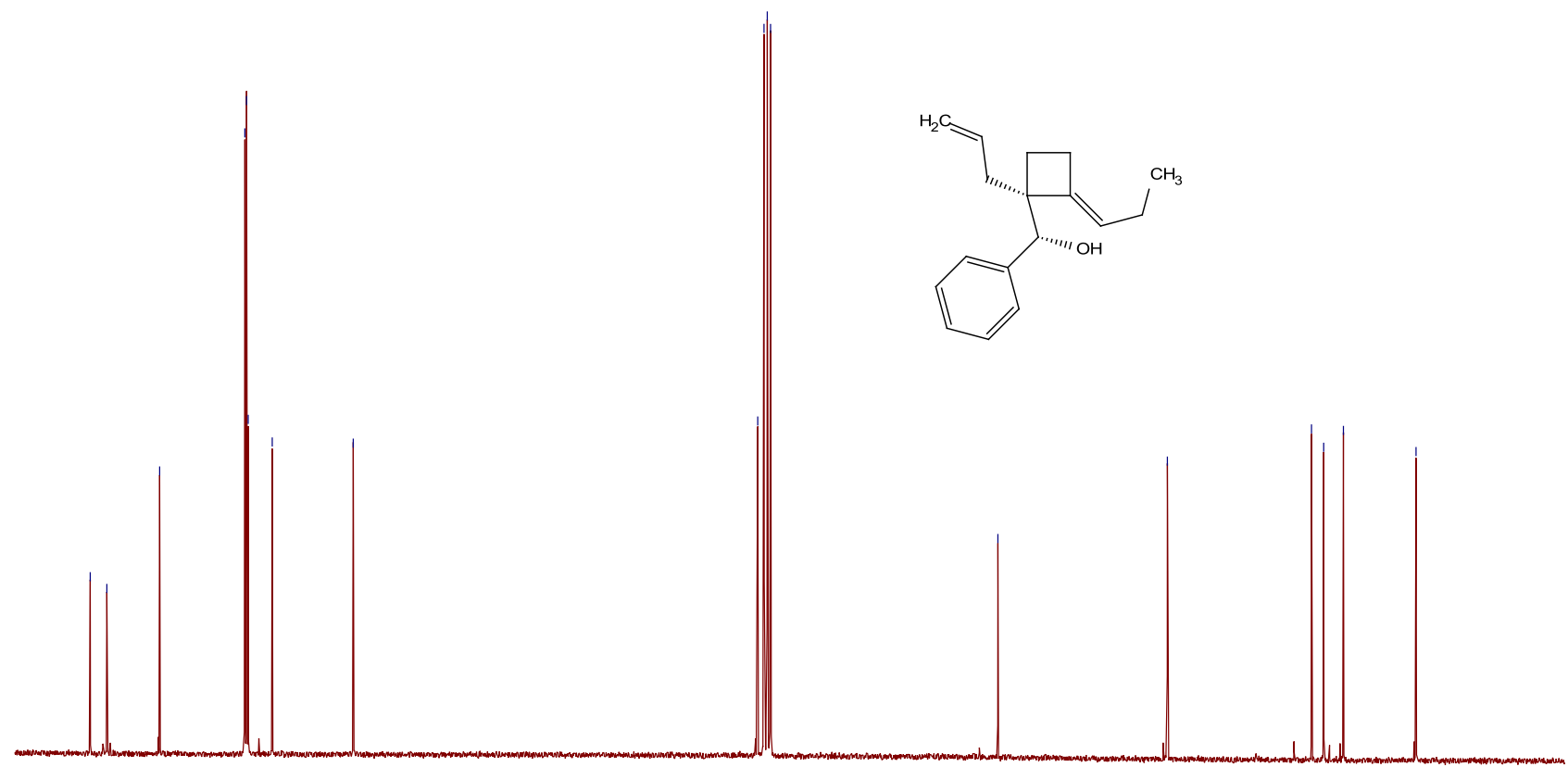

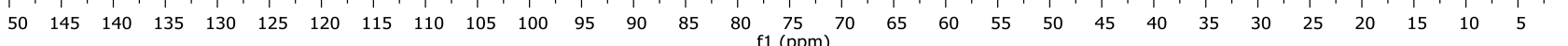


$\left(S^{*}\right)-\left(\left(R^{*}, E\right)\right.$-1-allyl-2-propylidenecyclobutyl)(2-chloro-6-fluorophenyl)methanol (4c)

${ }^{1} \mathrm{H} \mathrm{NMR}, 400 \mathrm{MHz}, \mathrm{CDCl}_{3}$

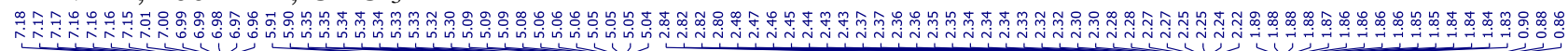
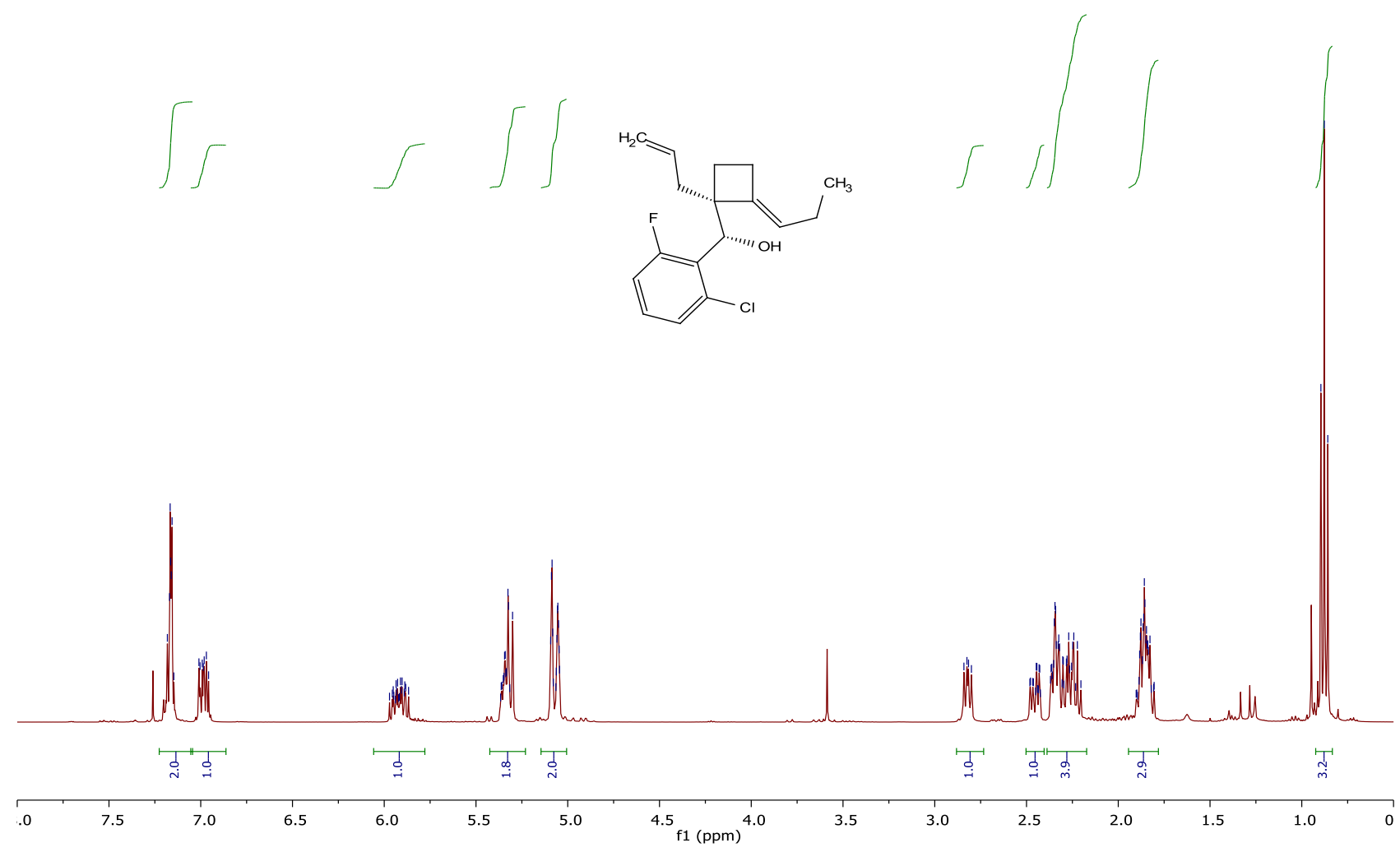

${ }^{13} \mathrm{C}$ NMR, $101 \mathrm{MHz}, \mathrm{CDCl}_{3}$

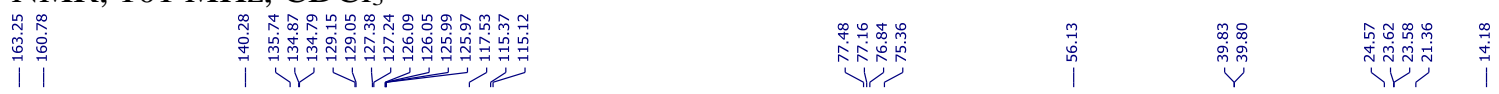

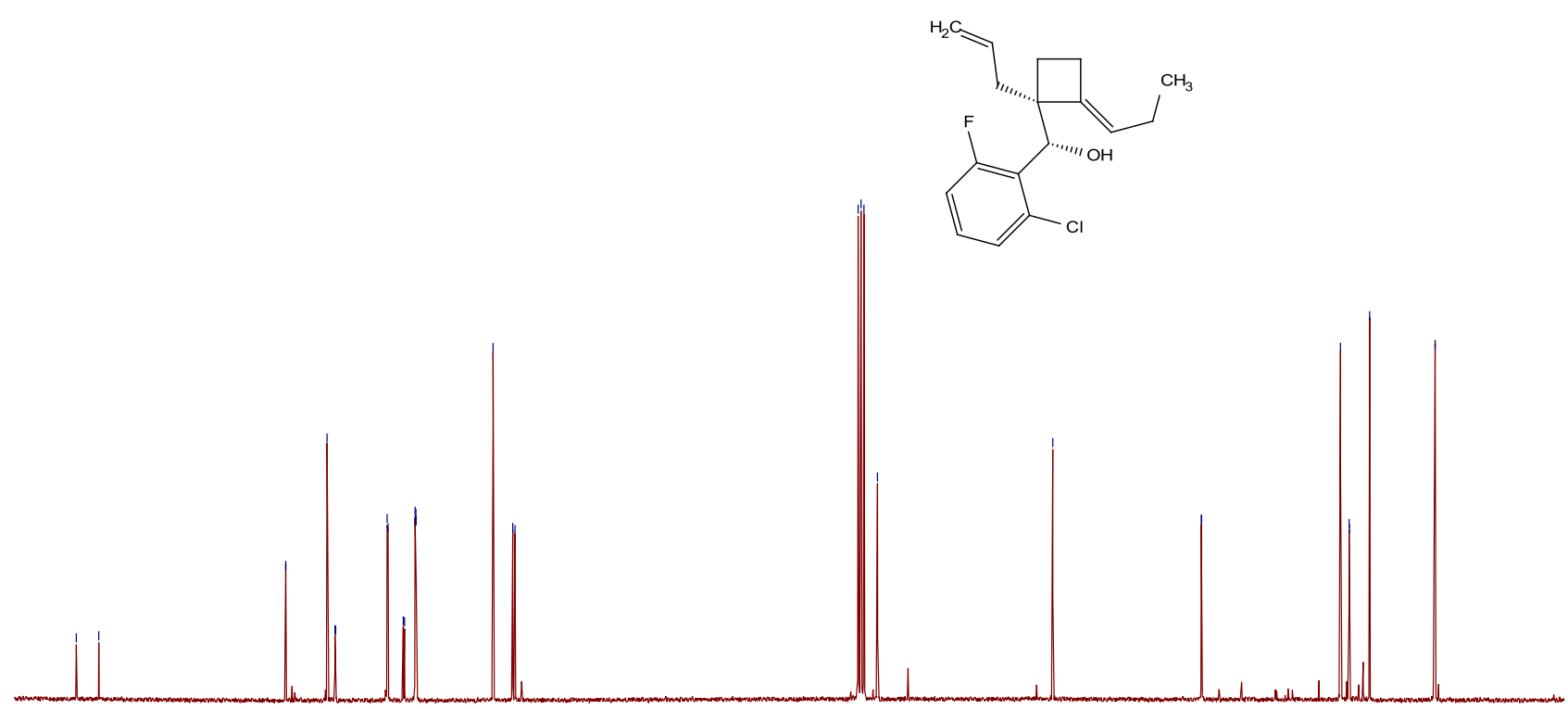

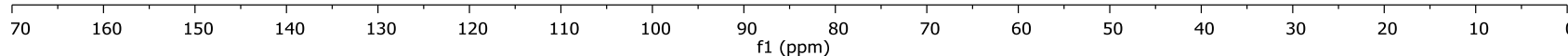


$\left(S^{*}\right)-\left(\left(R^{*}, E\right)-1\right.$-allyl-2-propylidenecyclobutyl)(5-bromopyridin-3-yl)methanol (4d)

${ }^{1} \mathrm{H} \mathrm{NMR}, 400 \mathrm{MHz}, \mathrm{CDCl}_{3}$

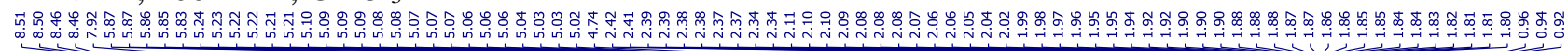

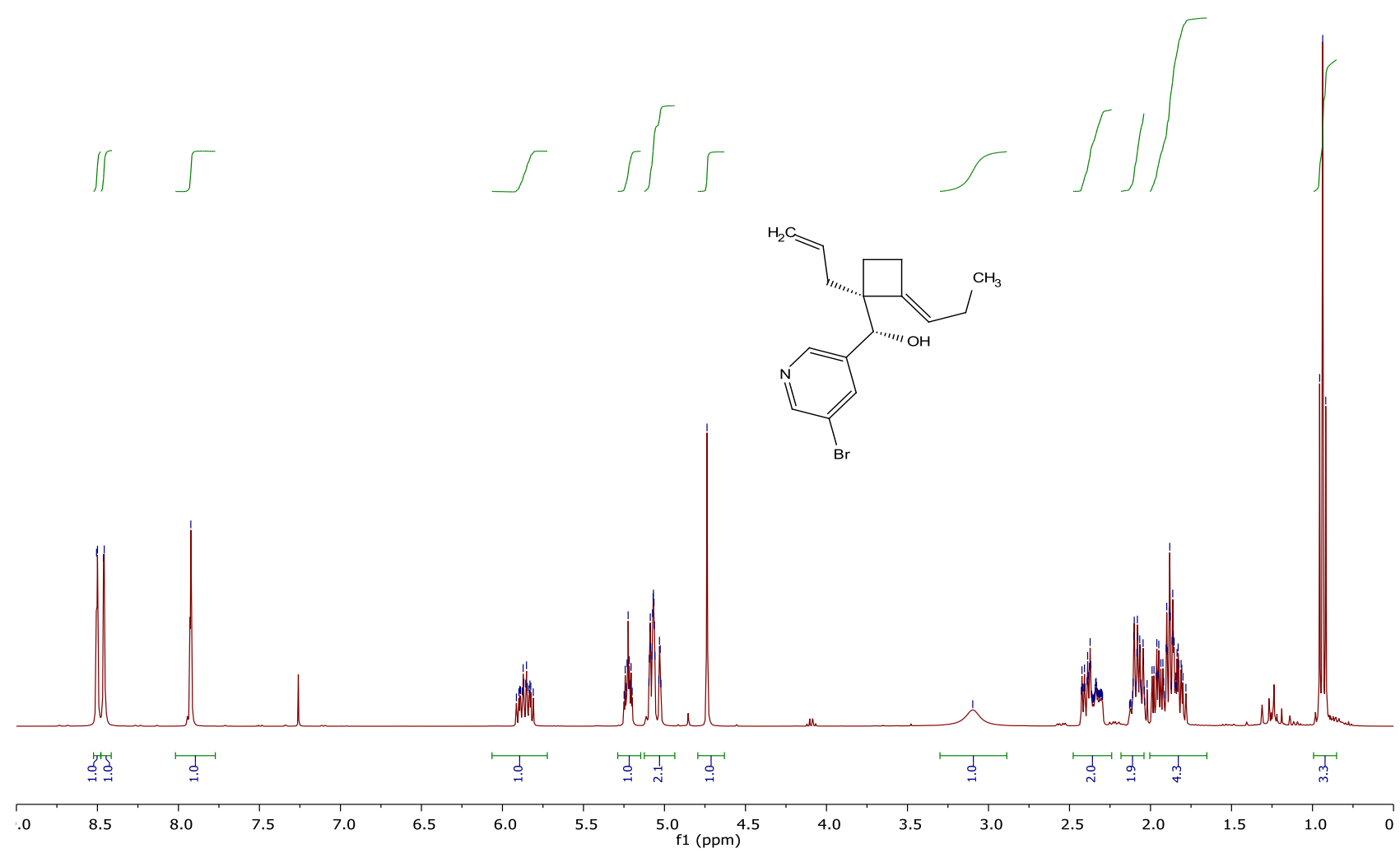

${ }^{13} \mathrm{C} \mathrm{NMR}, 101 \mathrm{MHz}, \mathrm{CDCl}_{3}$

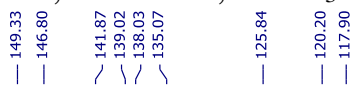

$\underbrace{-1}$

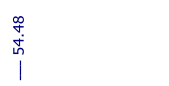

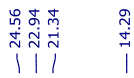
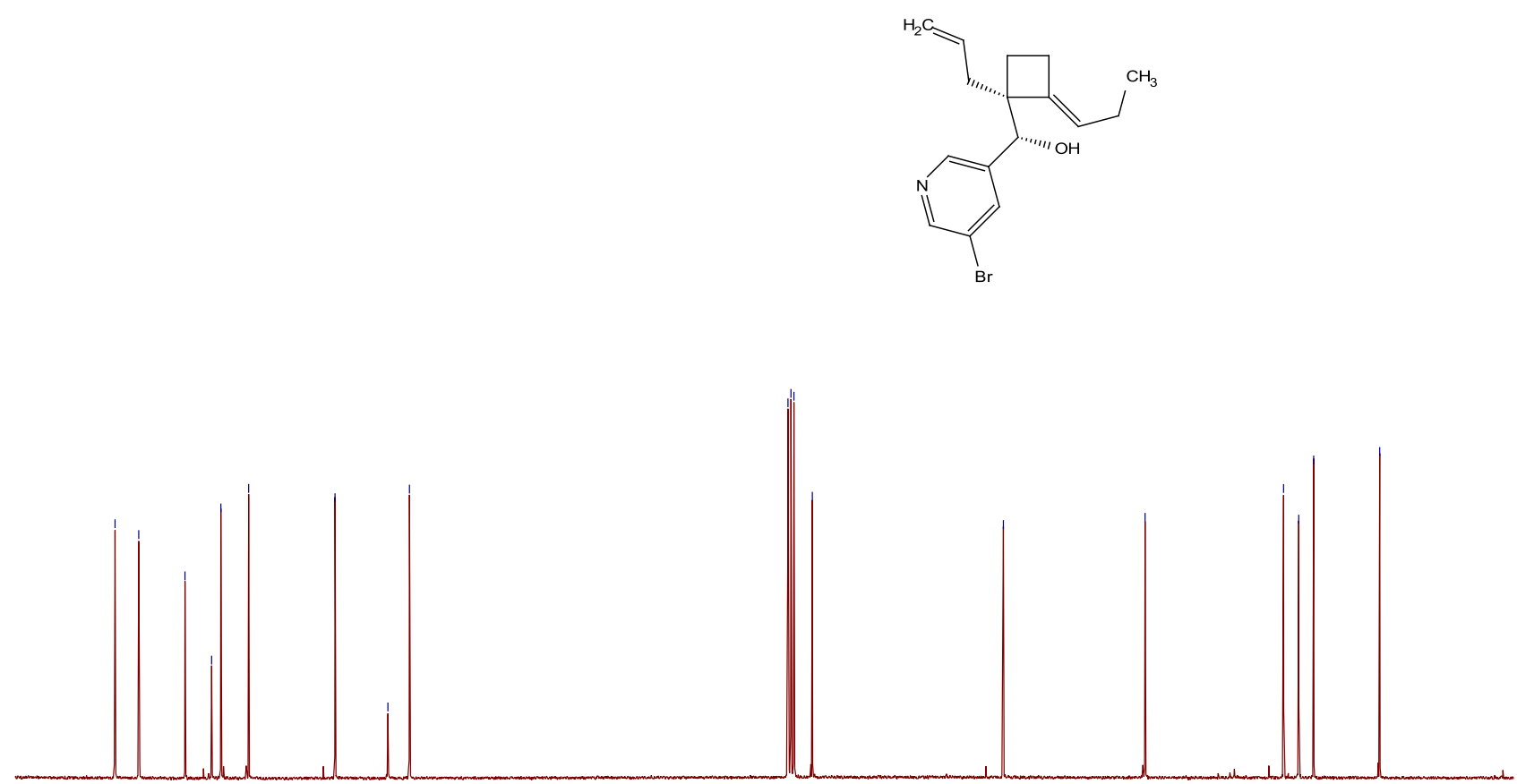

60

$150 \quad 140$

$120 \quad 110$

100

90

80

60

$\begin{array}{lll}\mid & & \mid \\ 40 & 20 & 10\end{array}$


$\left(S^{*}\right)-\left(\left(R^{*}, E\right)\right.$-1-allyl-2-propylidenecyclobutyl)(3-fluoro-6-methoxyquinolin-4-yl)methanol (4e)

${ }^{1} \mathrm{H} \mathrm{NMR}, 400 \mathrm{MHz}, \mathrm{CDCl}_{3}$

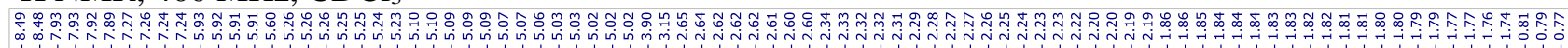

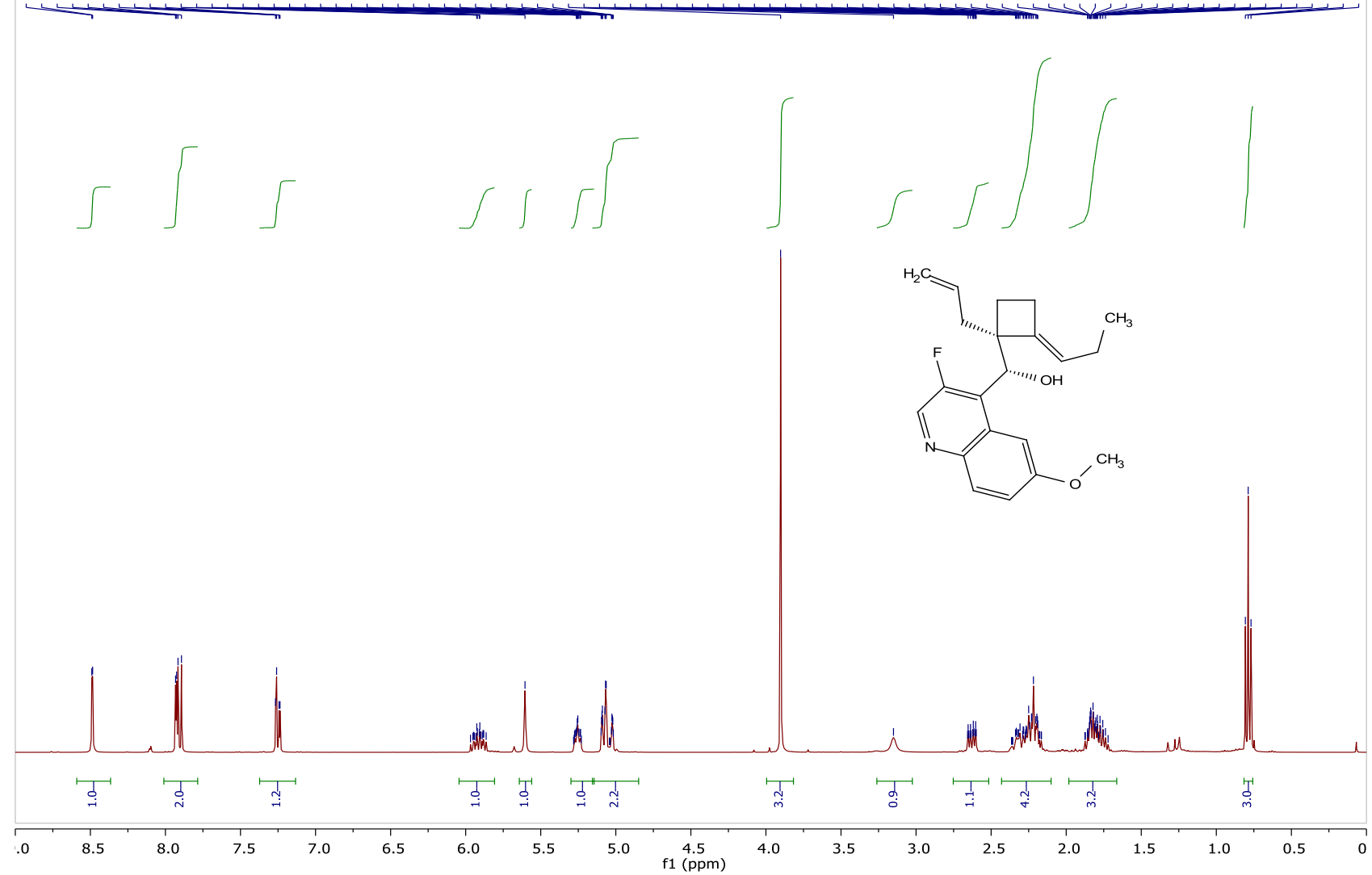

${ }^{13} \mathrm{C}$ NMR, $101 \mathrm{MHz}, \mathrm{CDCl}_{3}$

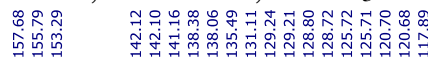

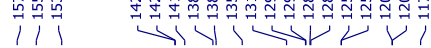

Y

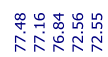

ilivis

ำ

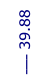

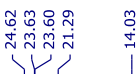

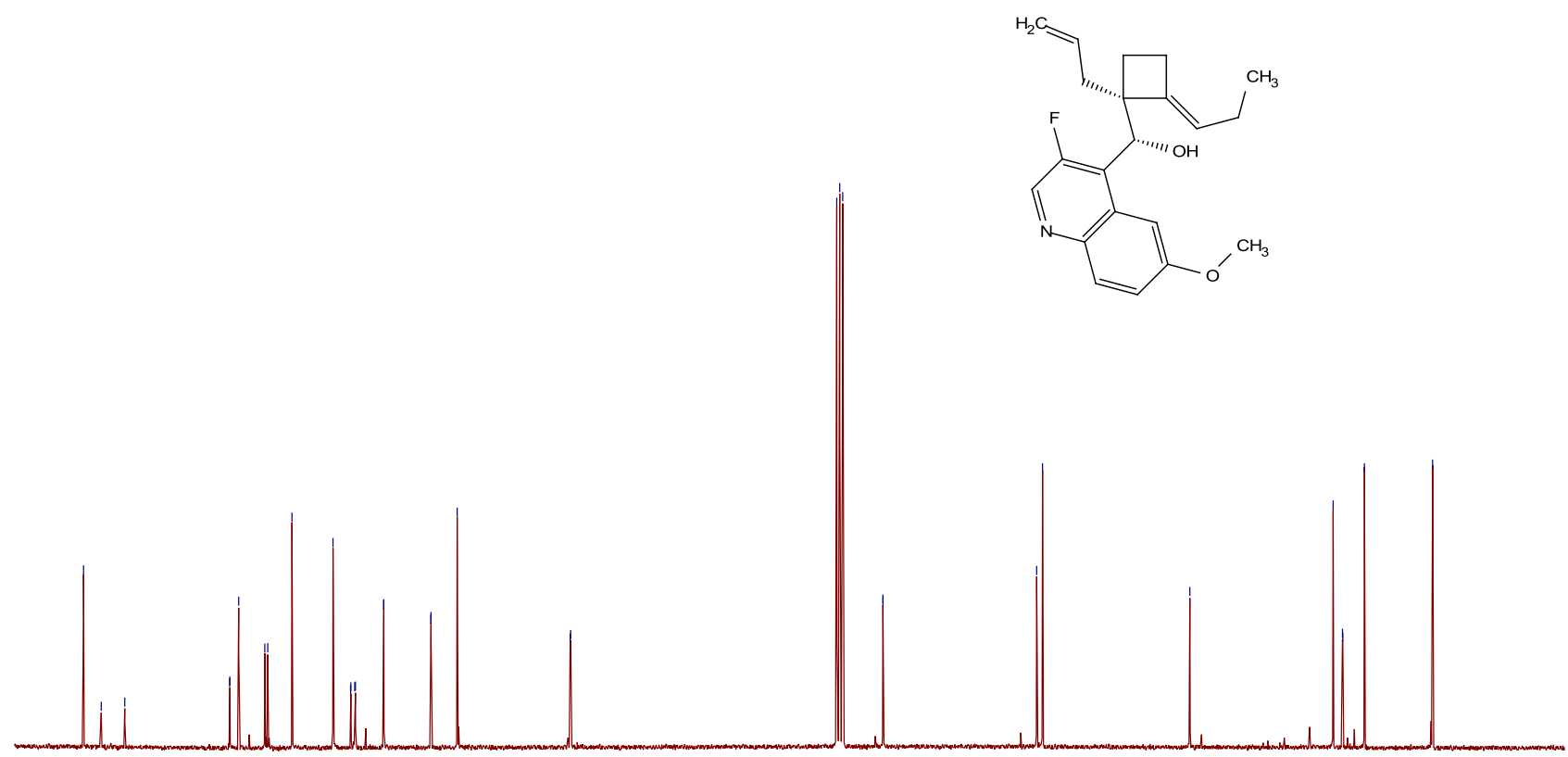

$160 \quad 150$

$140 \quad 130$

$120 \quad 110 \quad 100$

90

80
$(\mathrm{ppm})$

70

$60 \quad 50$ 
$\left(R^{*}\right)-1-\left(\left(R^{*}, E\right)-1\right.$-allyl-2-propylidenecyclobutyl)-3-phenylpropan-1-ol (4f)

${ }^{1} \mathrm{H} \mathrm{NMR}, 400 \mathrm{MHz}, \mathrm{CDCl}_{3}$

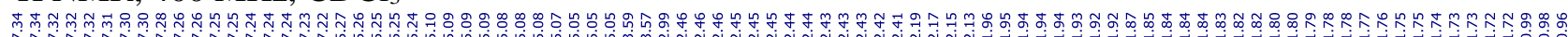
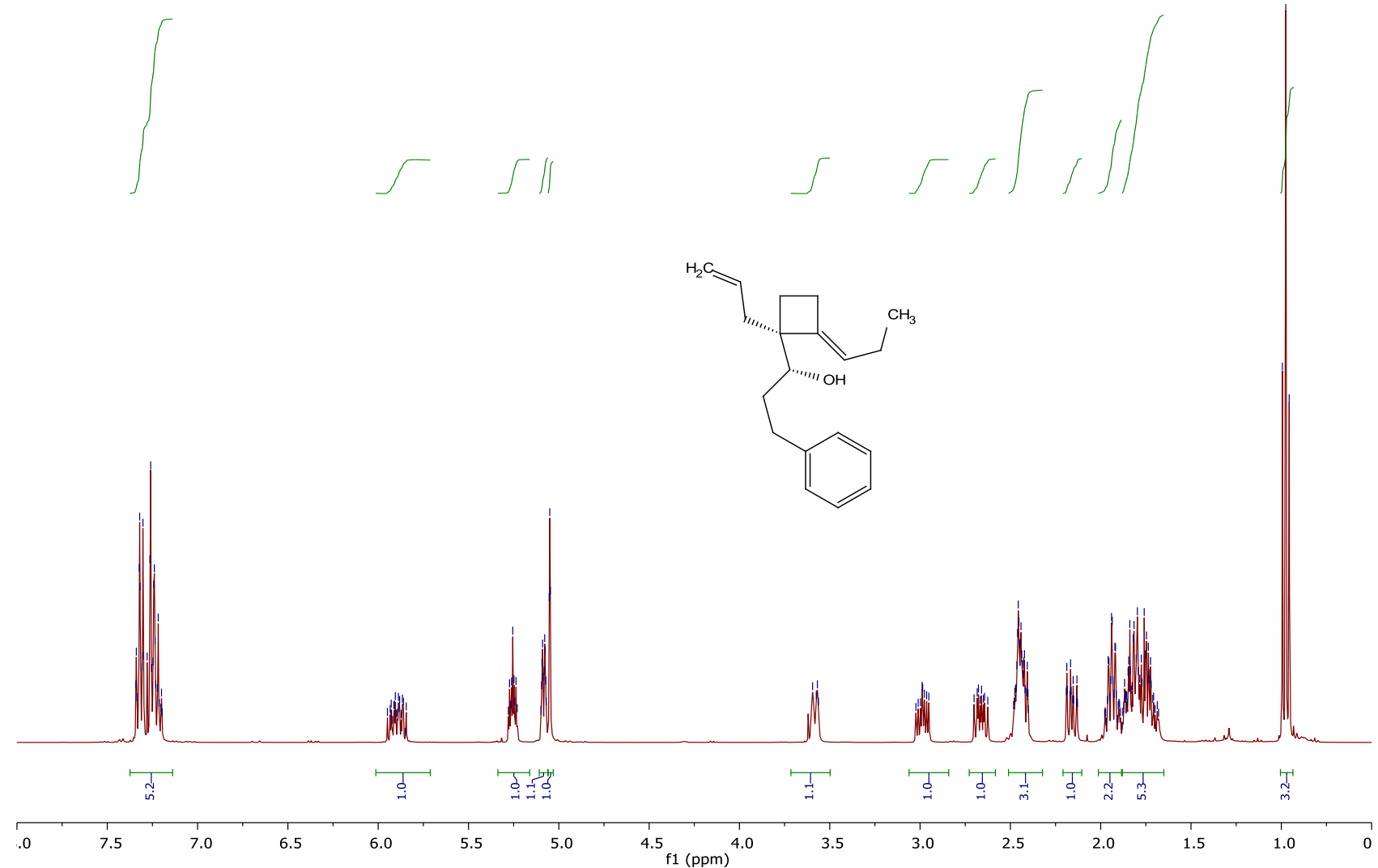

${ }^{13} \mathrm{C}$ NMR, $101 \mathrm{MHz}, \mathrm{CDCl}_{3}$

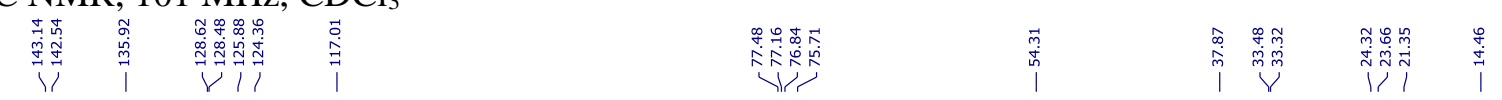
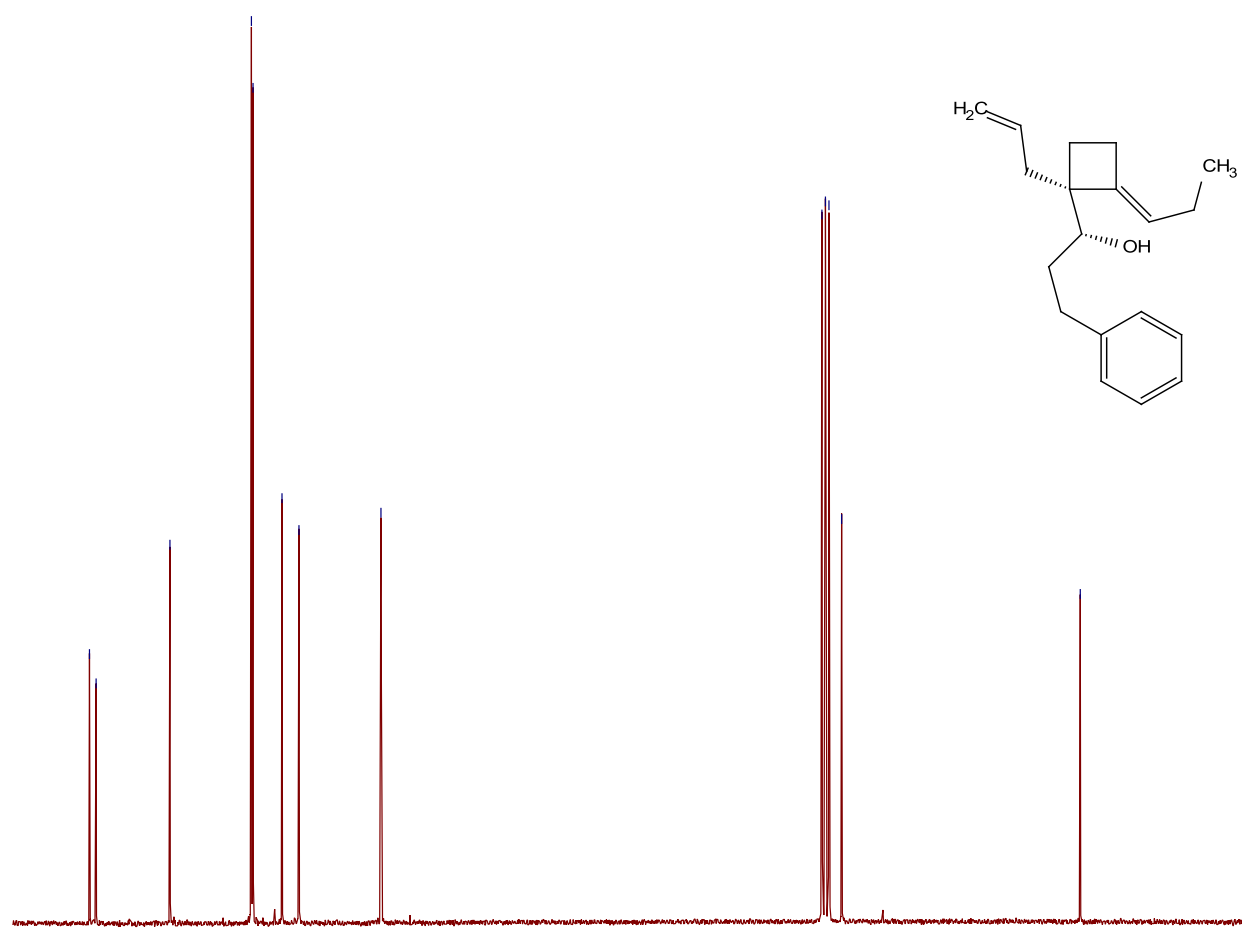

$\begin{array}{llllllllllllllllllllllllllllllllllllllll}50 & 145 & 140 & 135 & 130 & 125 & 120 & 115 & 110 & 105 & 100 & 95 & 90 & 85 & 80 & 75 & 70 & 65 & 60 & 55 & 50 & 45 & 40 & 35 & 30 & 25 & 20 & 15 & 10 & 5 & 15\end{array}$ 
$\left(R^{*}\right)-\left(\left(R^{*}, E\right)-1-a l l y l-2-(2-m e t h y l p r o p y l i d e n e) c y c l o b u t y l\right)($ phenyl)methanol (4g)

${ }^{1} \mathrm{H} \mathrm{NMR}, 400 \mathrm{MHz}, \mathrm{CDCl}_{3}$

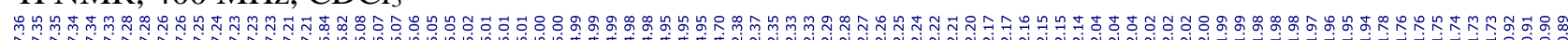

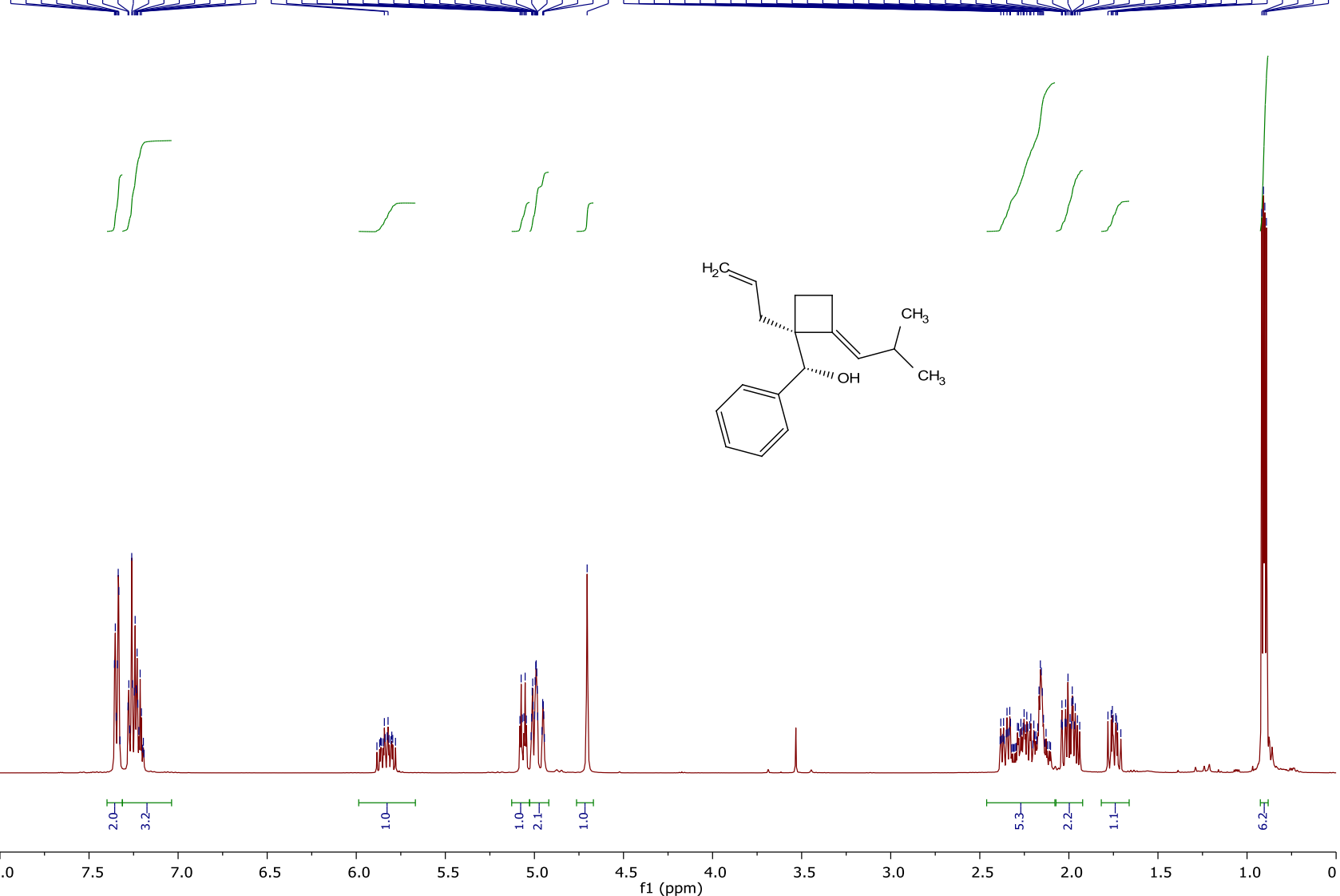

${ }^{13} \mathrm{C} \mathrm{NMR}, 101 \mathrm{MHz}, \mathrm{CDCl}_{3}$

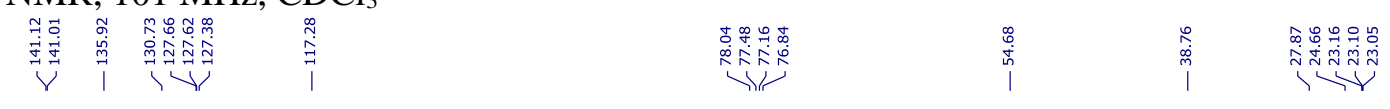

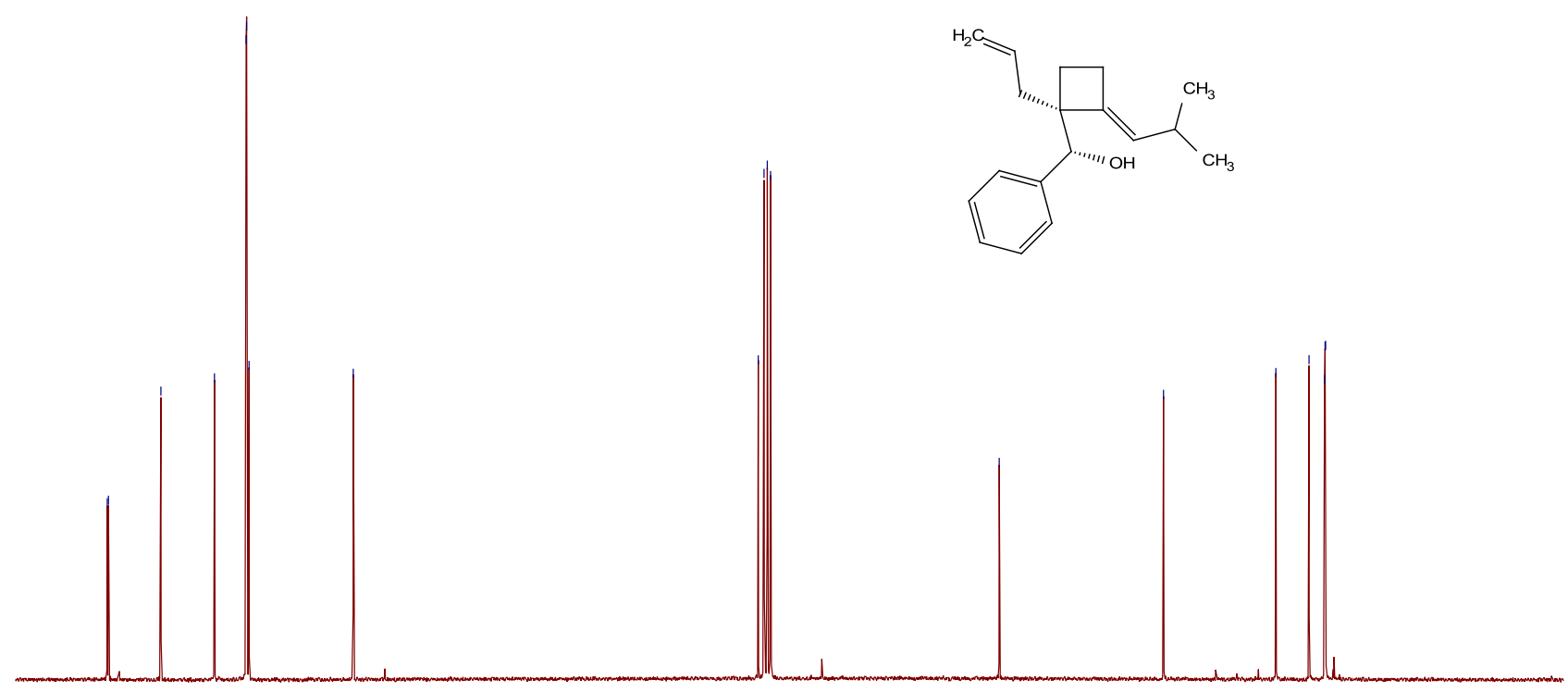

$\begin{array}{llllllllllllllllllllllllllllllllllllllll}50 & 145 & 140 & 135 & 130 & 125 & 120 & 115 & 110 & 105 & 100 & 95 & 90 & 85 & 80 & 75 & 70 & 65 & 60 & 55 & 50 & 45 & 40 & 35 & 30 & 25 & 20 & 15 & 10 & 5 & 1\end{array}$ 
$\left(S^{*}\right)-\left(\left(R^{*}, E\right)\right.$-1-allyl-2-(2-methylpropylidene)cyclobutyl)(2-chloro-6-fluorophenyl)methanol (4h)

${ }^{1} \mathrm{H} \mathrm{NMR}, 400 \mathrm{MHz}, \mathrm{CDCl}_{3}$

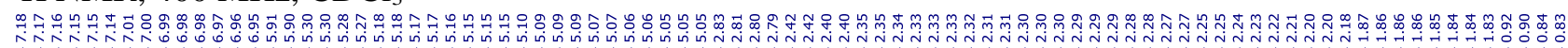

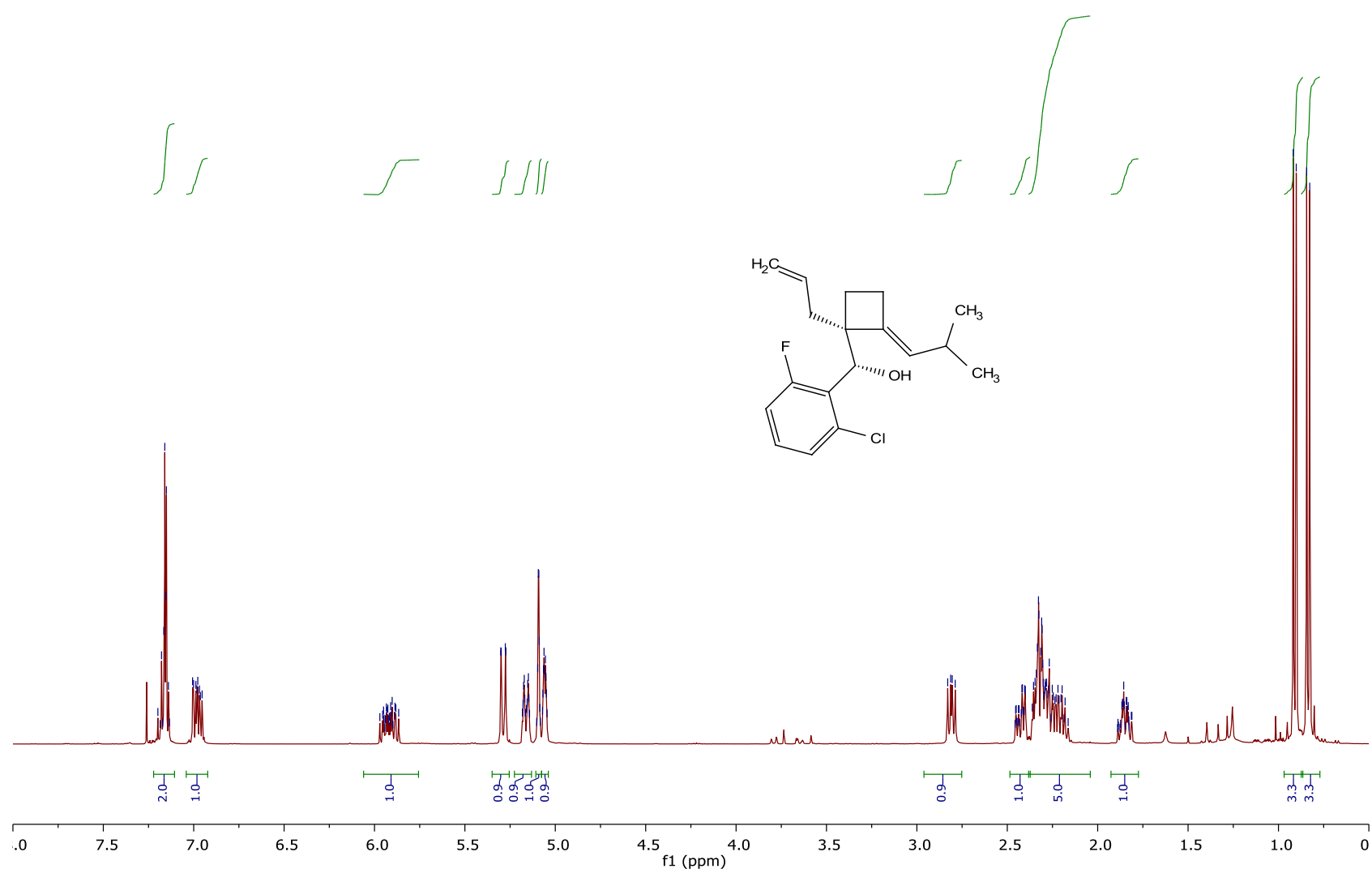

${ }^{13} \mathrm{C} \mathrm{NMR,} 101 \mathrm{MHz}, \mathrm{CDCl}_{3}$

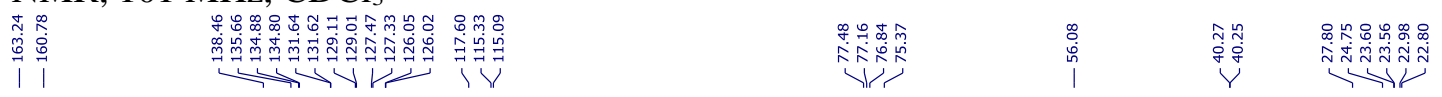

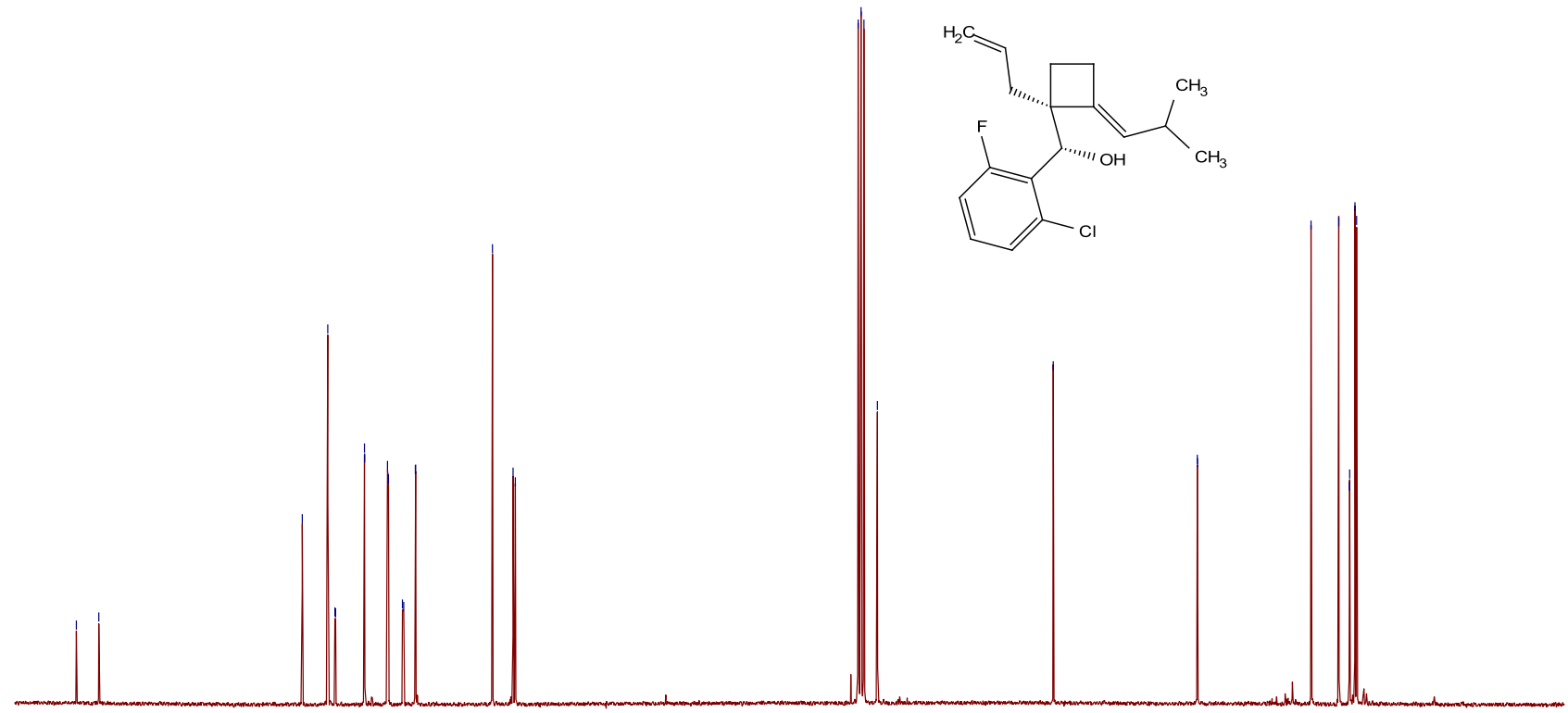

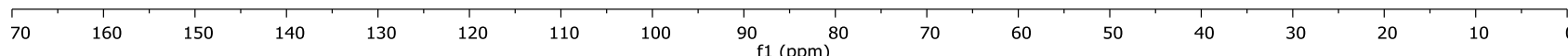


$\left(S^{*}\right)-\left(\left(R^{*}, E\right)\right.$-1-allyl-2-(2-methylpropylidene)cyclobutyl)(5-bromopyridin-3-yl)-methanol (4i)

${ }^{1} \mathrm{H} \mathrm{NMR}, 400 \mathrm{MHz}, \mathrm{CDCl}_{3}$

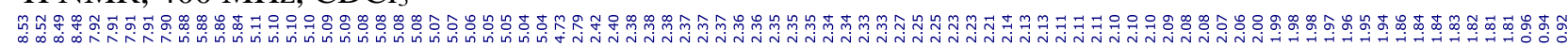

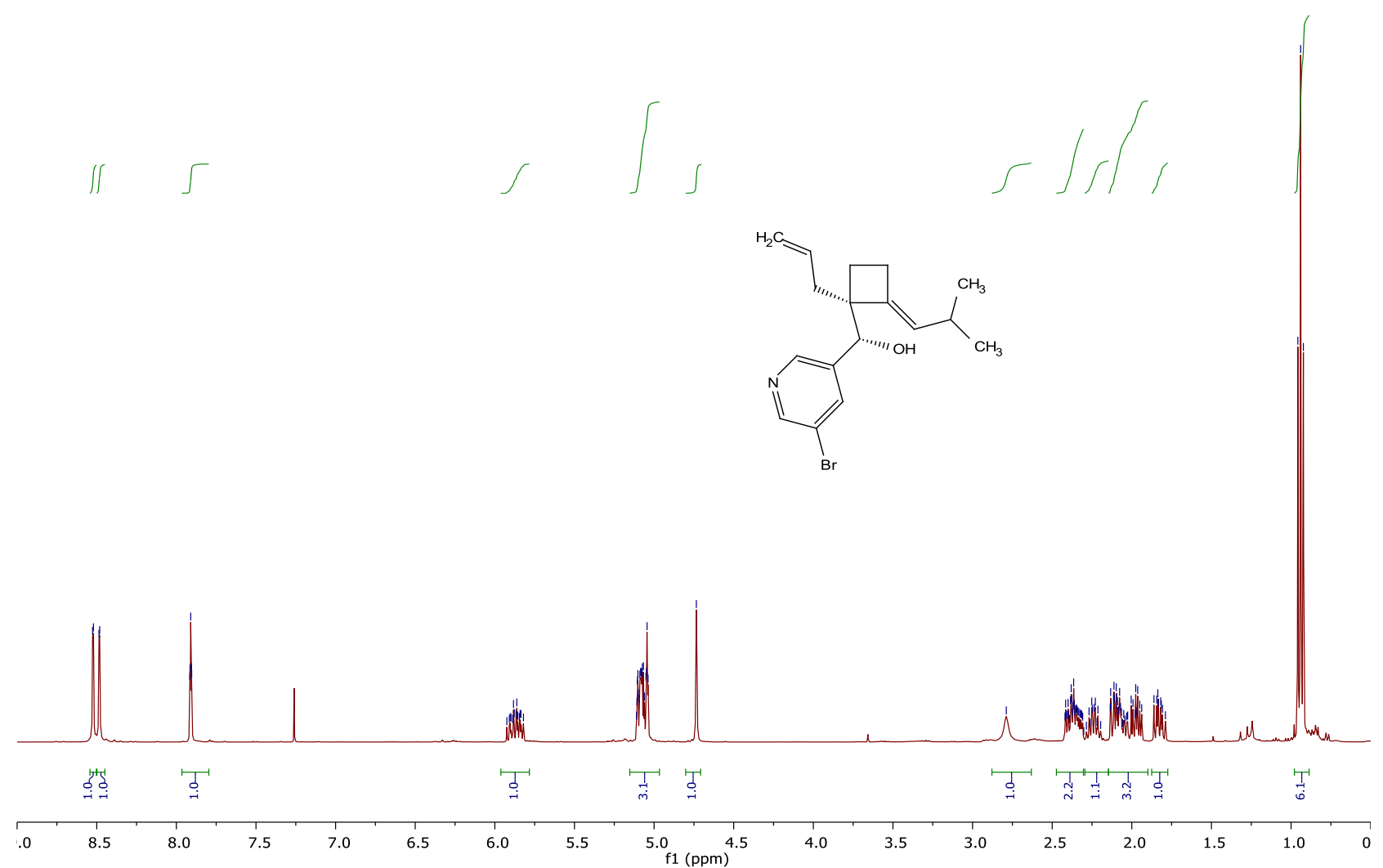

${ }^{13} \mathrm{C} \mathrm{NMR}, 101 \mathrm{MHz}, \mathrm{CDCl}_{3}$

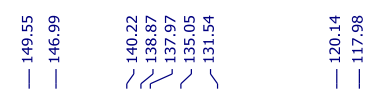

| |

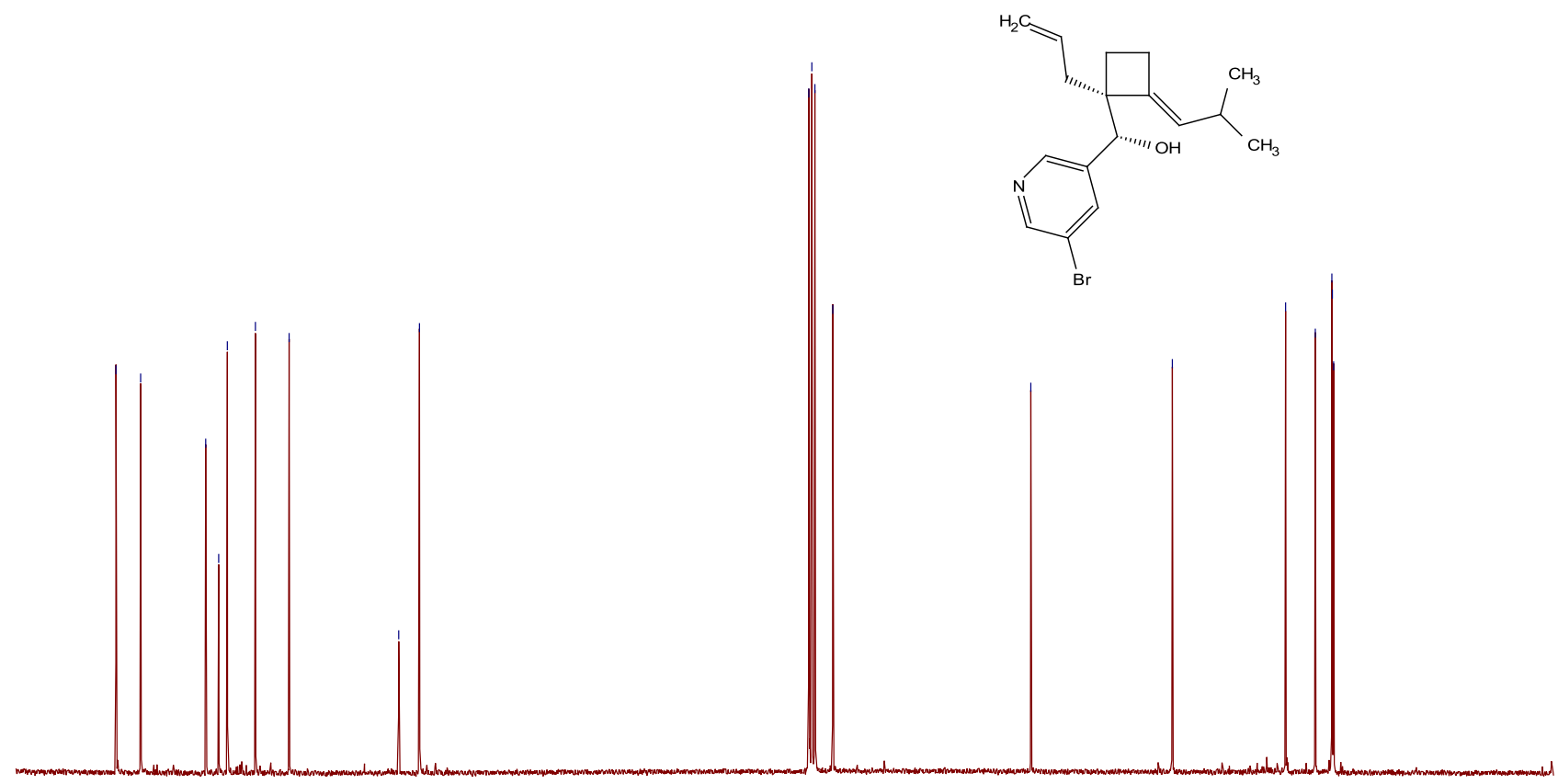

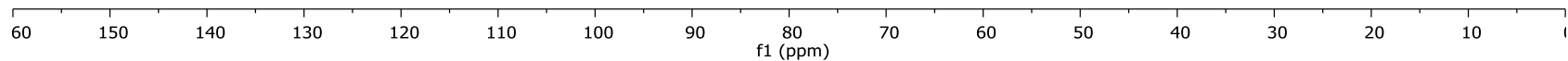




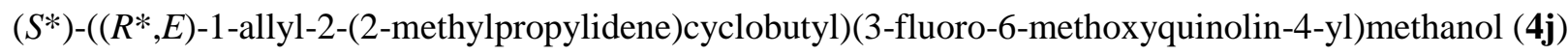
${ }^{1} \mathrm{H} \mathrm{NMR}, 400 \mathrm{MHz}, \mathrm{CDCl}_{3}$

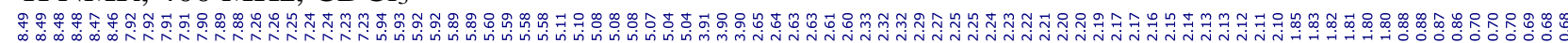

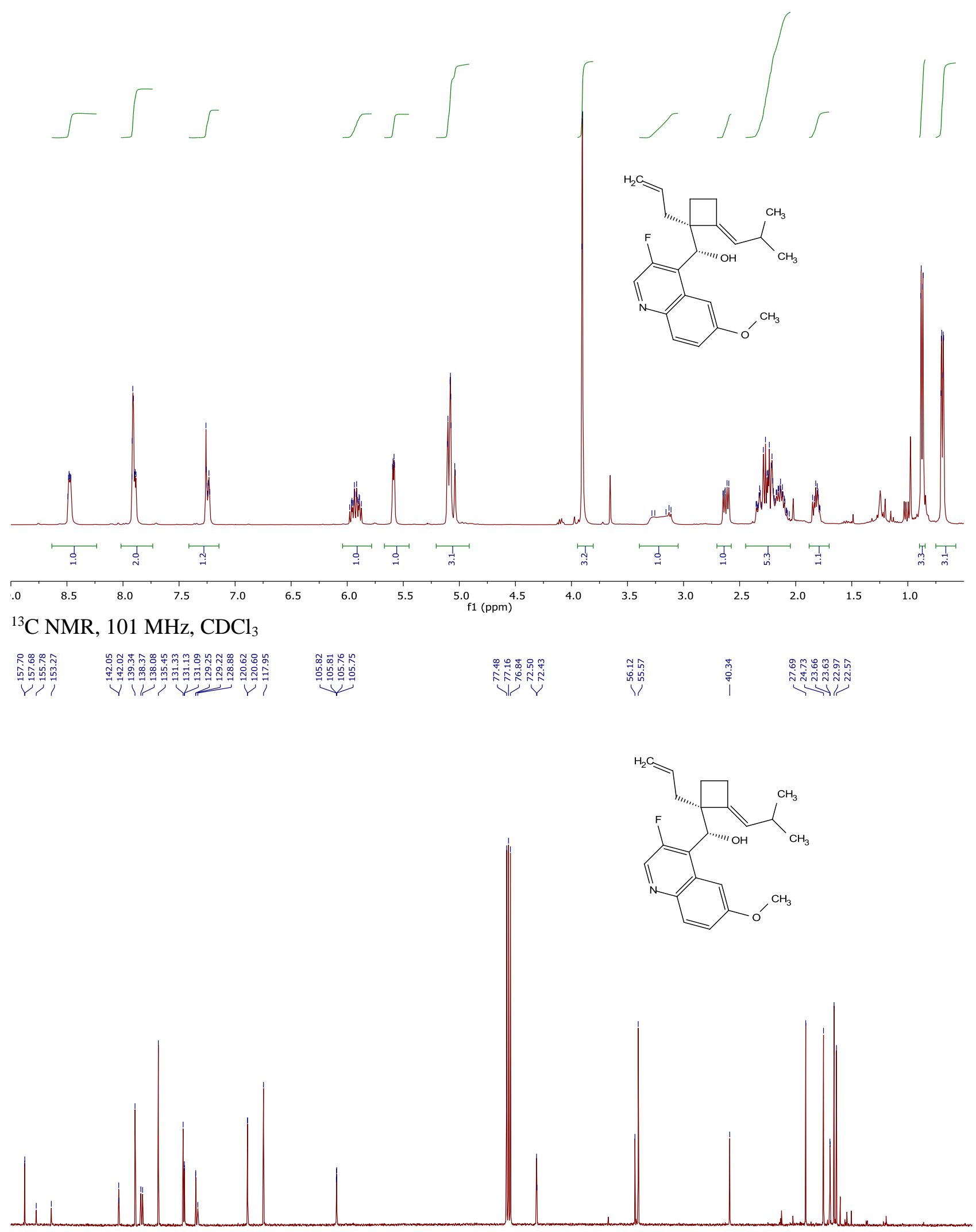

60
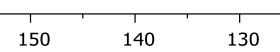

120

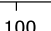

90

80
$\mathrm{f} 1(\mathrm{ppm})$ 
$\left(R^{*}\right)$-1-(( $\left.R^{*}, E\right)$-1-allyl-2-(2-methylpropylidene)cyclobutyl)-3-phenylpropan-1-ol (4k)

${ }^{1} \mathrm{H} \mathrm{NMR}, 400 \mathrm{MHz}, \mathrm{CDCl}_{3}$

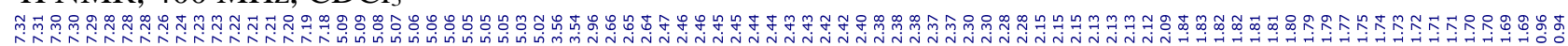
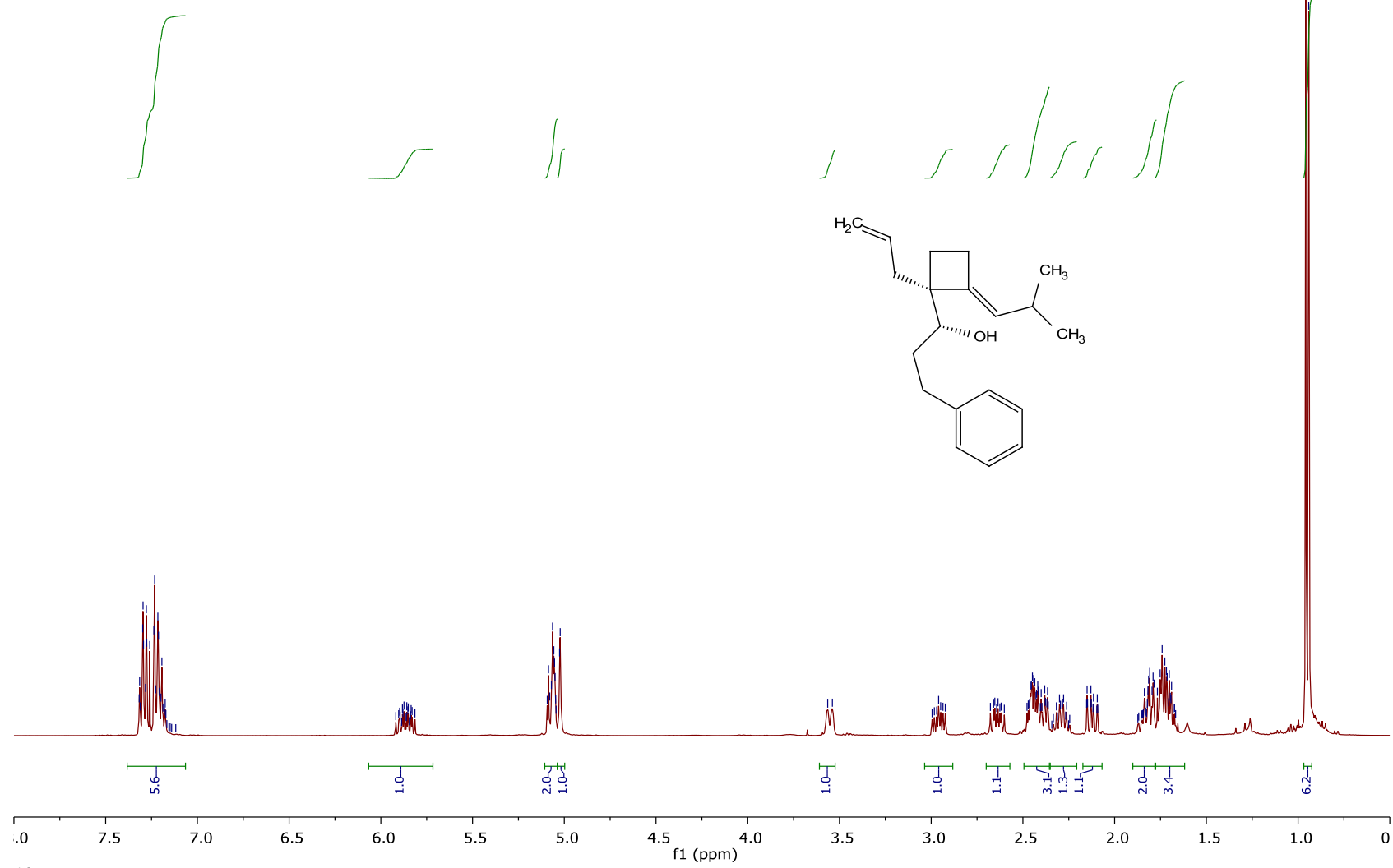

${ }^{13} \mathrm{C}$ NMR, $101 \mathrm{MHz}, \mathrm{CDCl}_{3}$

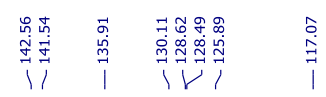

垈

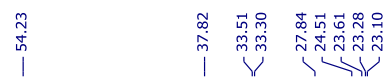
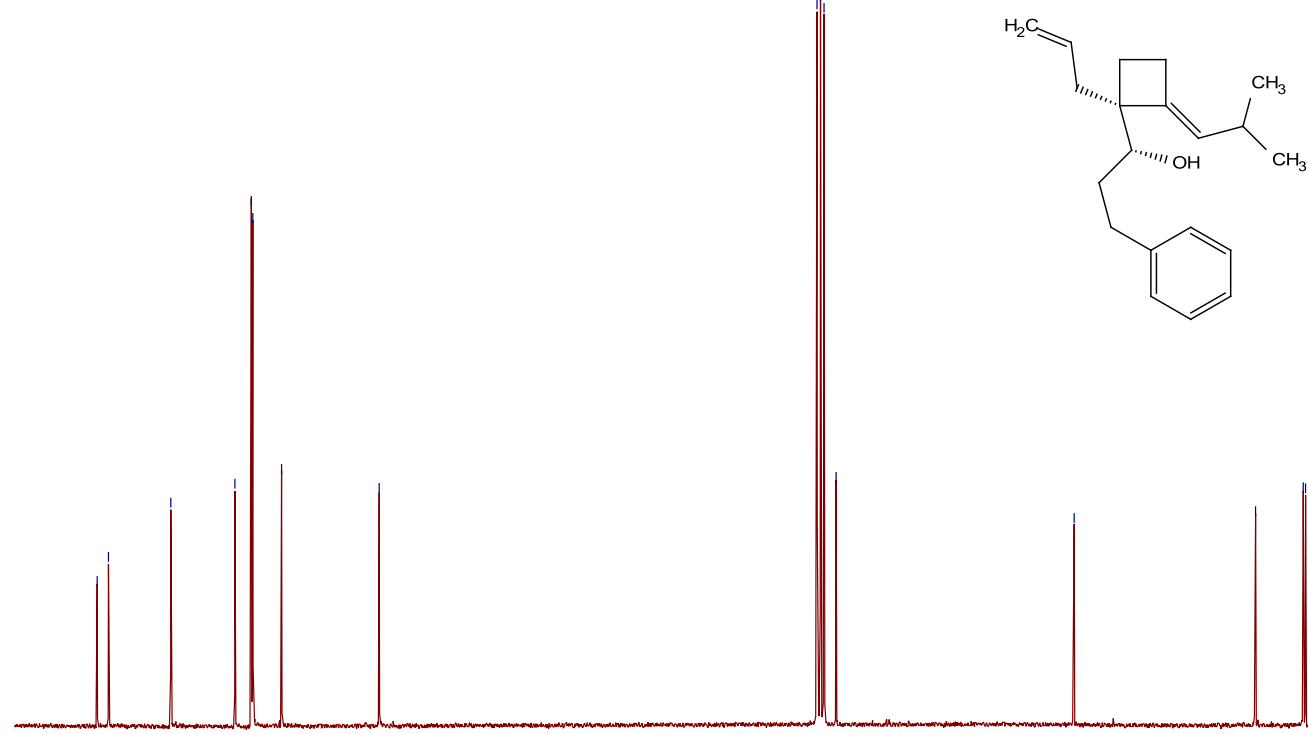

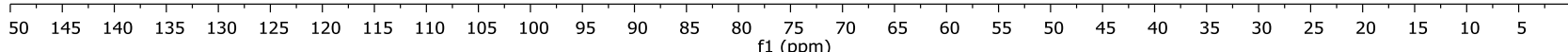


$\left(R^{*}\right)-\left(\left(R^{*}, E\right)\right.$-1-allyl-2-pentylidenecyclobutyl)(4-nitrophenyl)methanol (4I)

${ }^{1} \mathrm{H} \mathrm{NMR}, 400 \mathrm{MHz}, \mathrm{CDCl}_{3}$

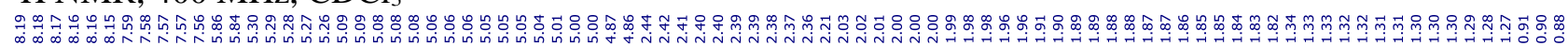

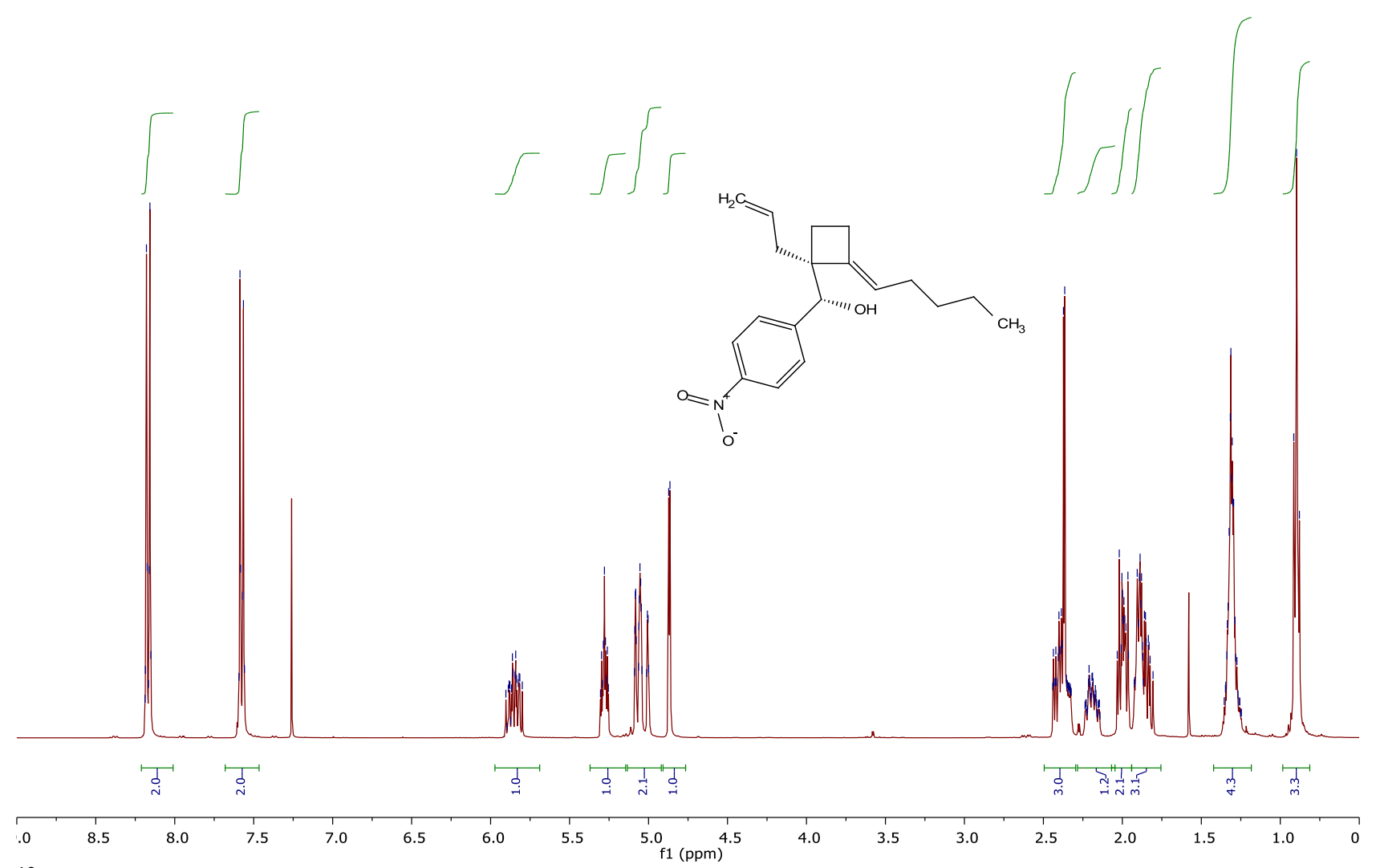

${ }^{13} \mathrm{C}$ NMR, $101 \mathrm{MHz}, \mathrm{CDCl}_{3}$

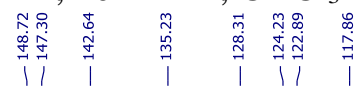

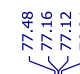

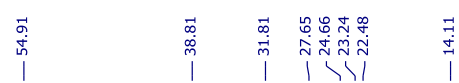
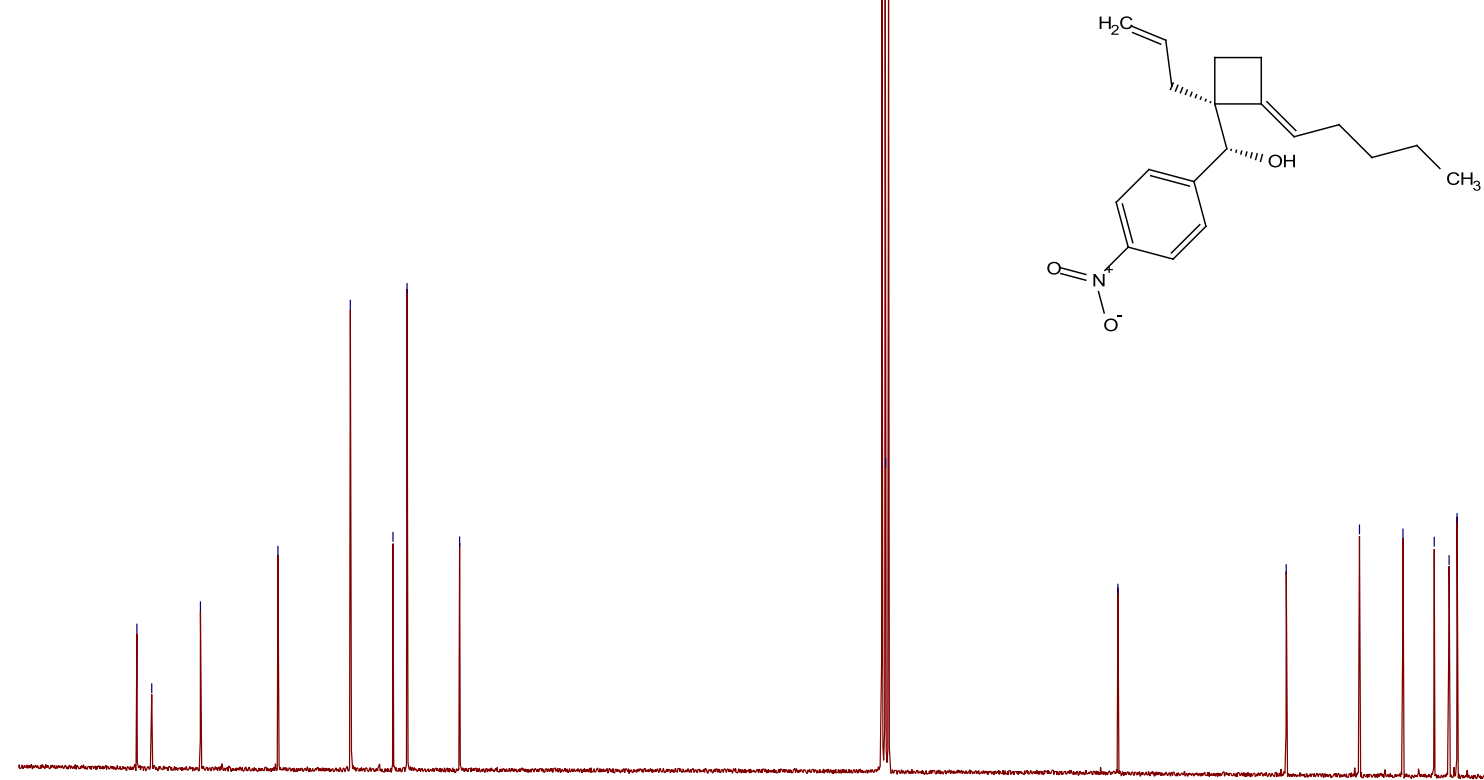

60

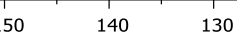

$30 \quad 120$

110

00

90

80
f1 $(p p m)$ 
$\left(S^{*}\right)$-[1,1'-biphenyl]-4-yl $\left(\left(S^{*}, E\right)\right.$-1-allyl-2-pentylidenecyclobutyl)methanol (4m)

${ }^{1} \mathrm{H} \mathrm{NMR}, 400 \mathrm{MHz}, \mathrm{CDCl}_{3}$
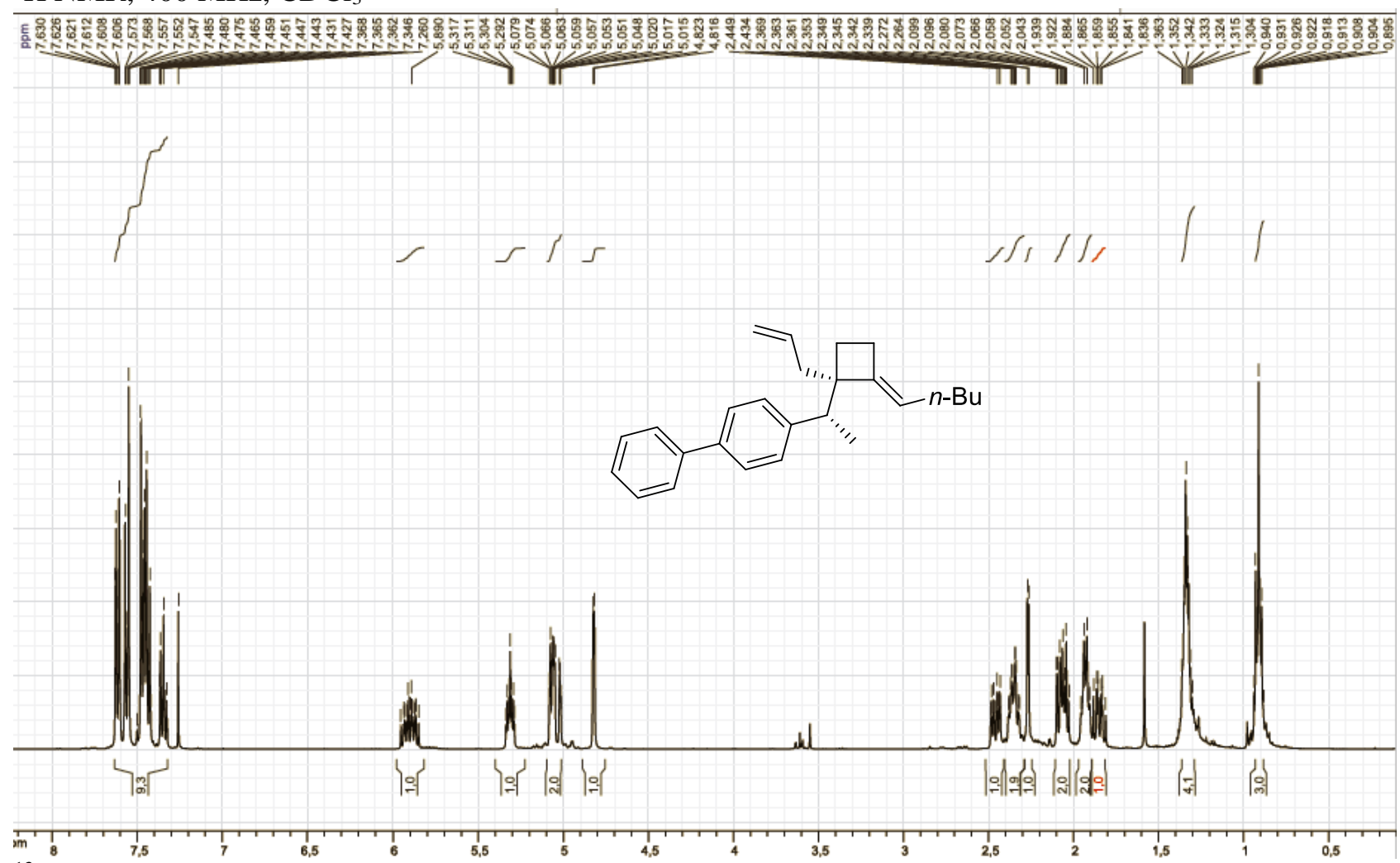

${ }^{13} \mathrm{C}^{8} \mathrm{NMR}, 101 \mathrm{MHz}^{7,5} \mathrm{CDCl}_{3}$
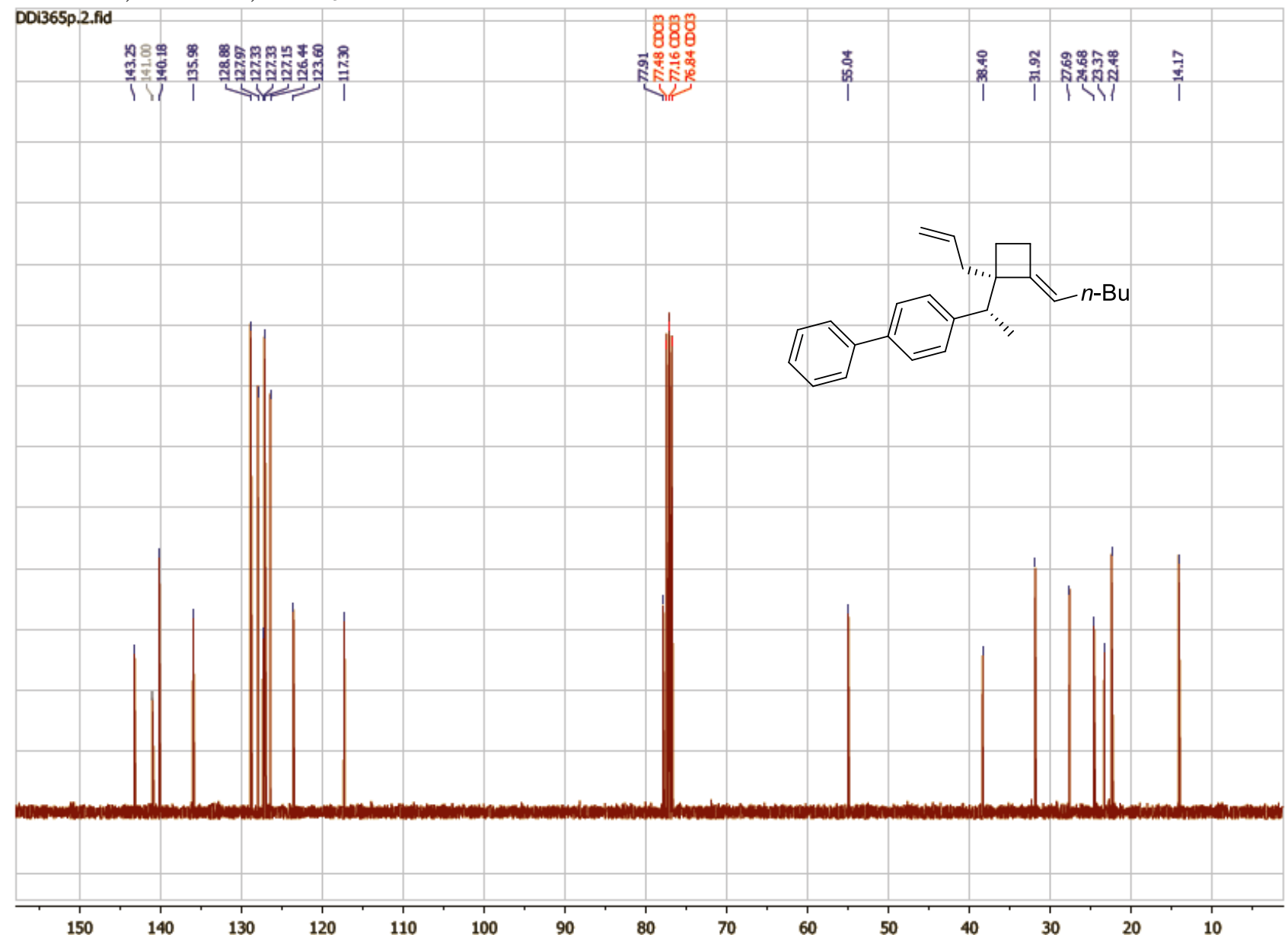
$\left(R^{*}\right)-\left(\left(S^{*}, E\right)\right.$-1-allyl-2-pentylidenecyclobutyl)(3-fluoro-6-methoxyquinolin-4-yl)methanol (4n)

${ }^{1} \mathrm{H} \mathrm{NMR}, 400 \mathrm{MHz}, \mathrm{CDCl}_{3}$
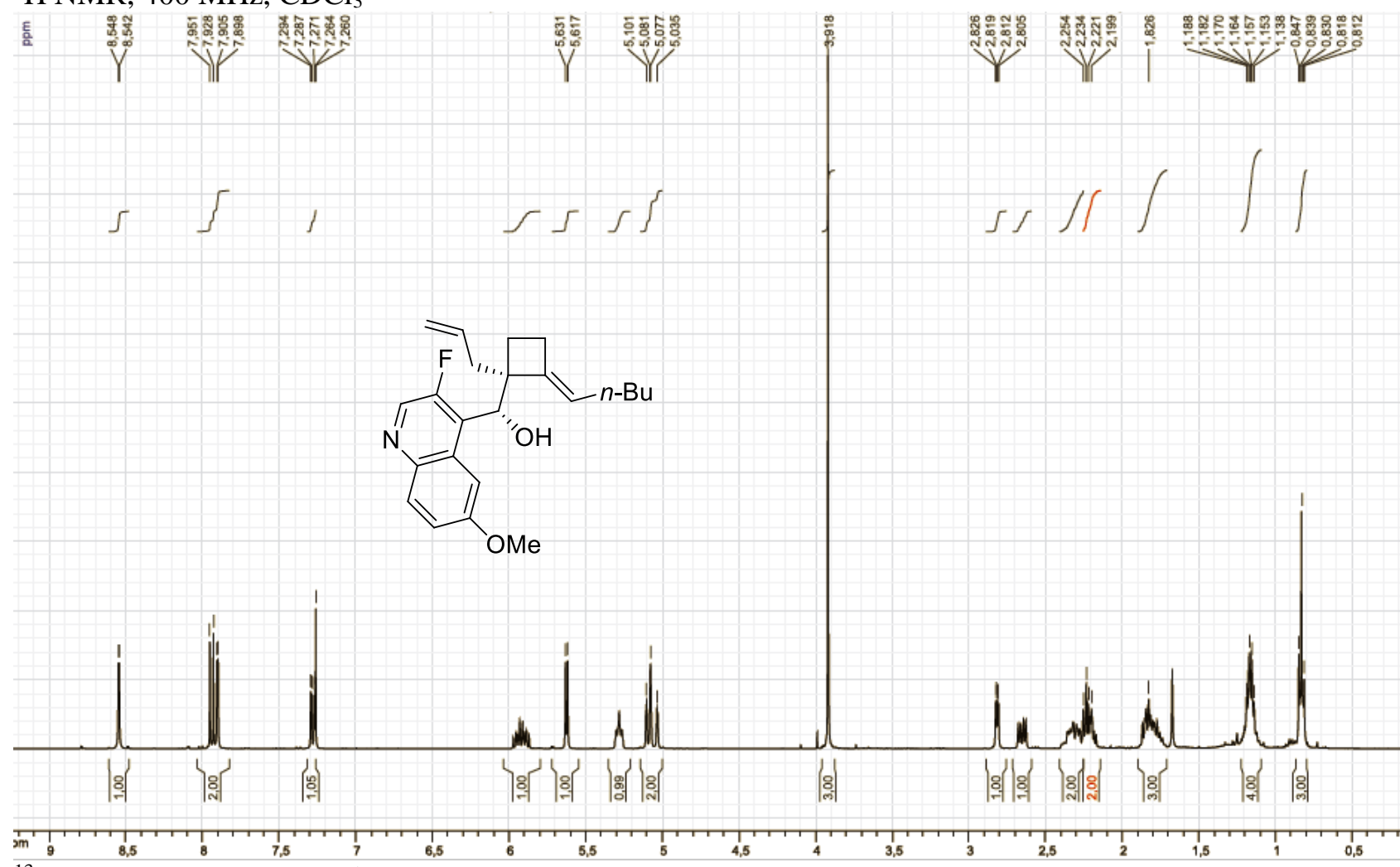

${ }^{13} \mathrm{C} \mathrm{NMR}, 101 \mathrm{MHz}, \mathrm{CDCl}_{3}$

DDi370p.2.fid

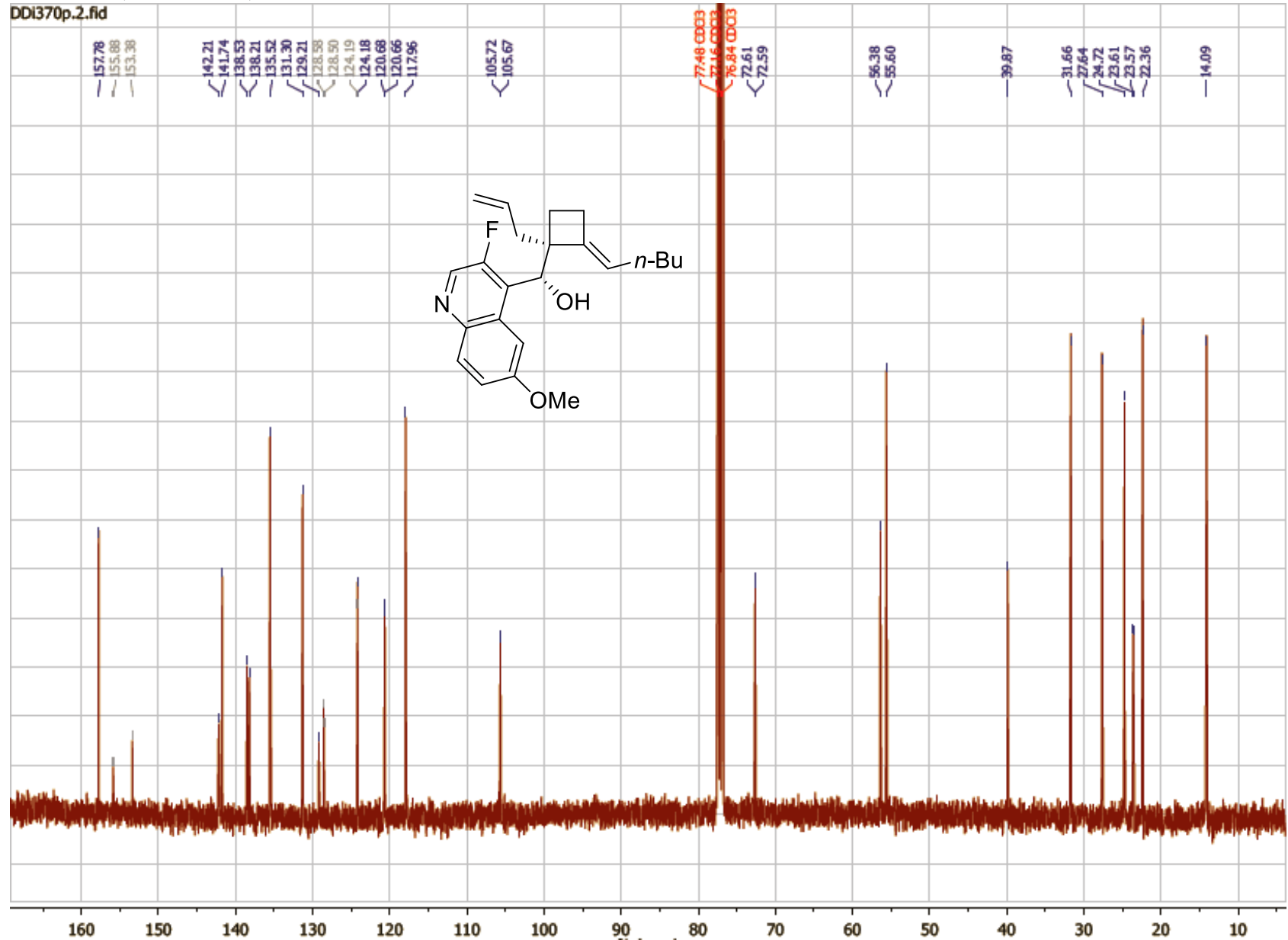


$\left(S^{*}\right)$-1-(( $\left.S^{*}, E\right)$-1-allyl-2-pentylidenecyclobutyl)-3-phenylpropan-1-ol (4o)

${ }^{1} \mathrm{H}$ NMR, $400 \mathrm{MHz}, \mathrm{CDCl}_{3}$

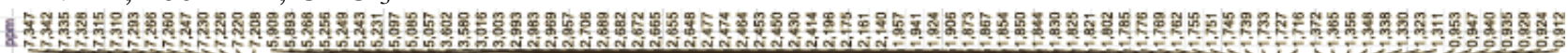
IIII Tा

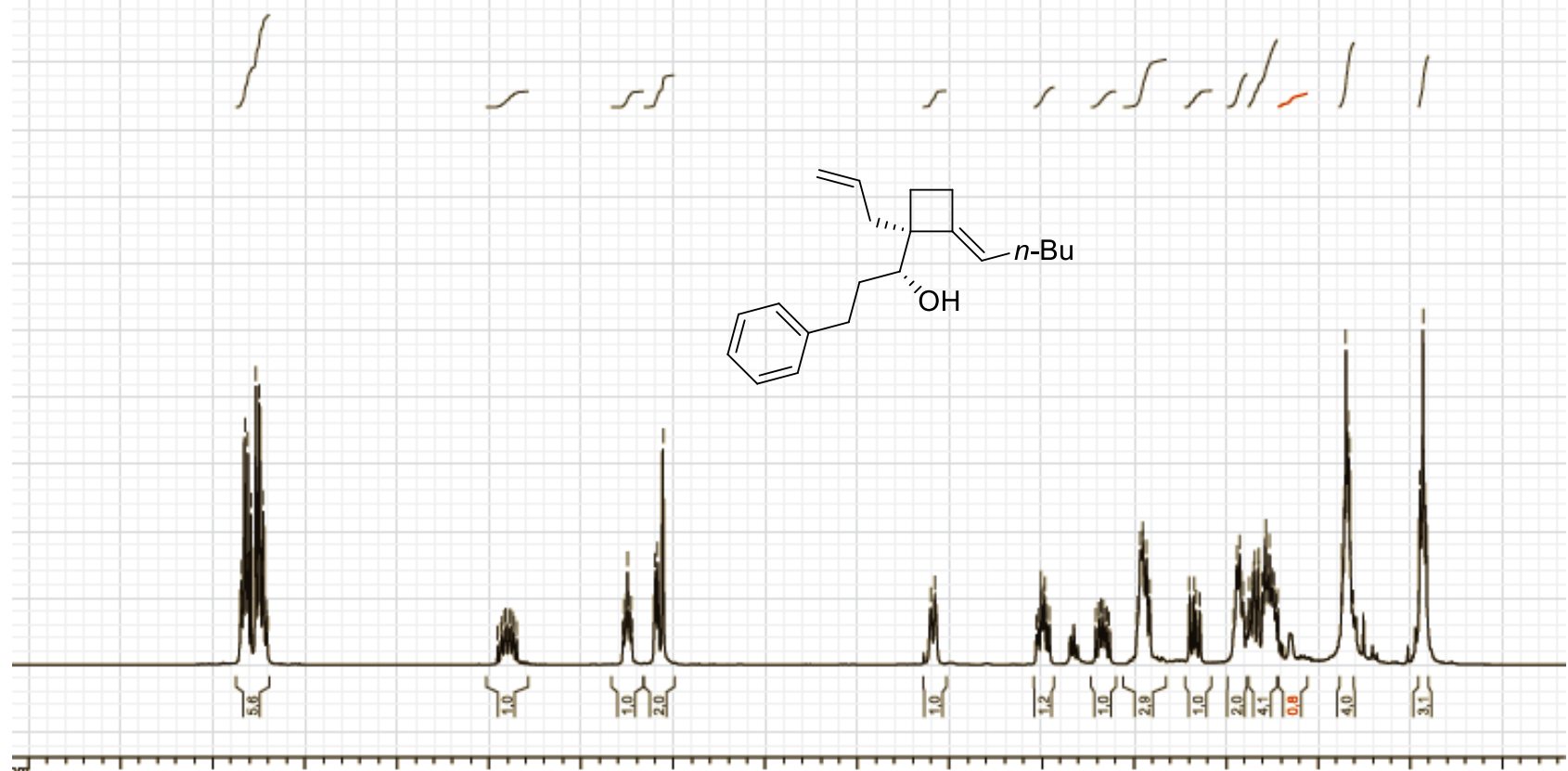

${ }^{13} \mathrm{C}$ NMR, $101 \mathrm{MHz}, \mathrm{CDCl}_{3}$

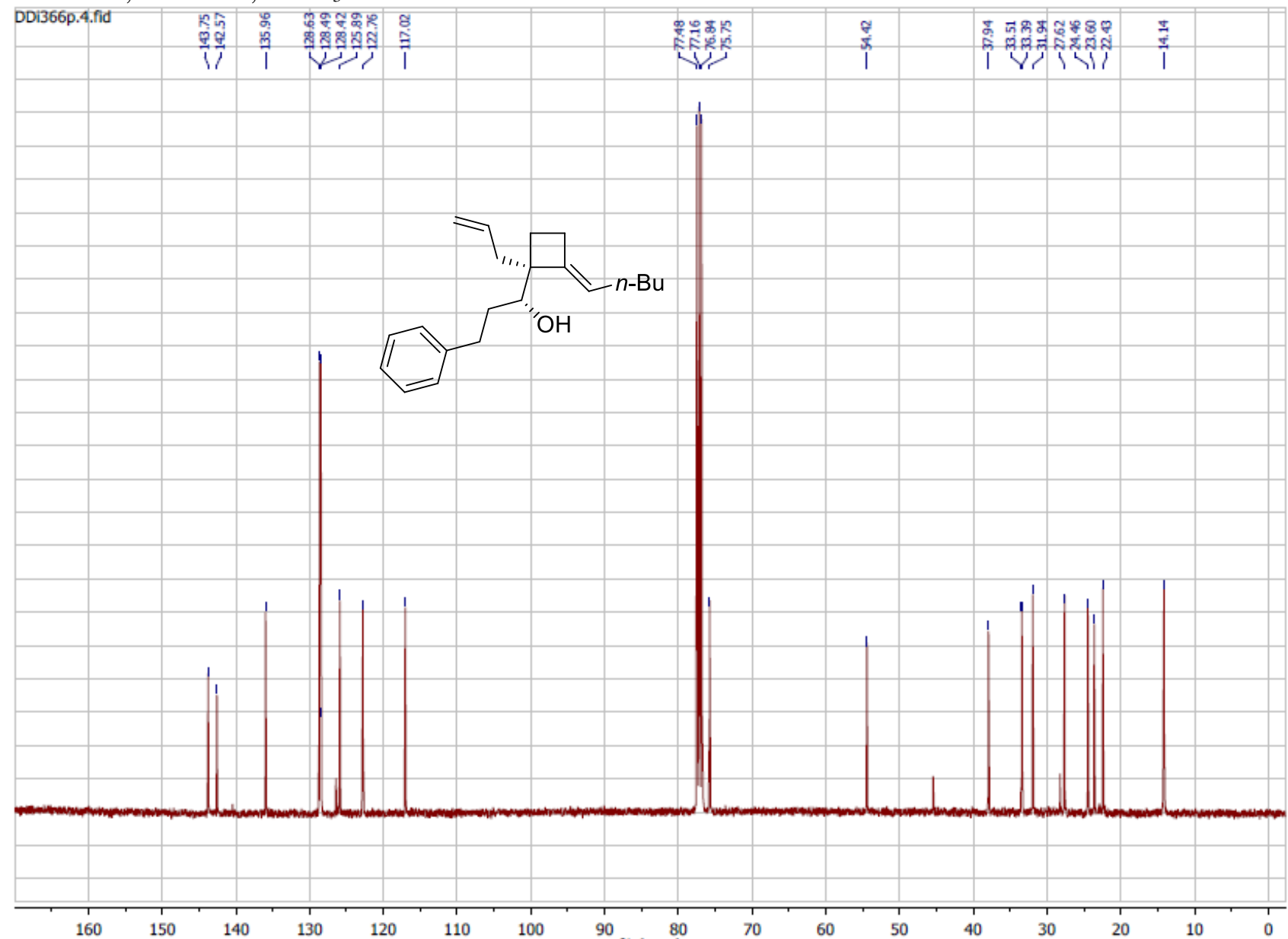


$\left(S^{*}\right)$-(2-bromopyridin-3-yl) $\left(\left(R^{*}, E\right)\right.$-1-(2-methylallyl)-2-(2-methylpropylidene)cyclobutyl)methanol (4p)

${ }^{1} \mathrm{H} \mathrm{NMR}, 400 \mathrm{MHz}, \mathrm{CDCl}_{3}$

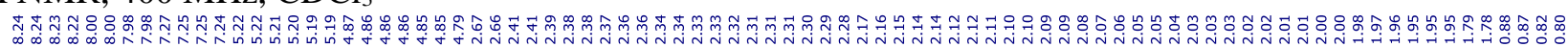

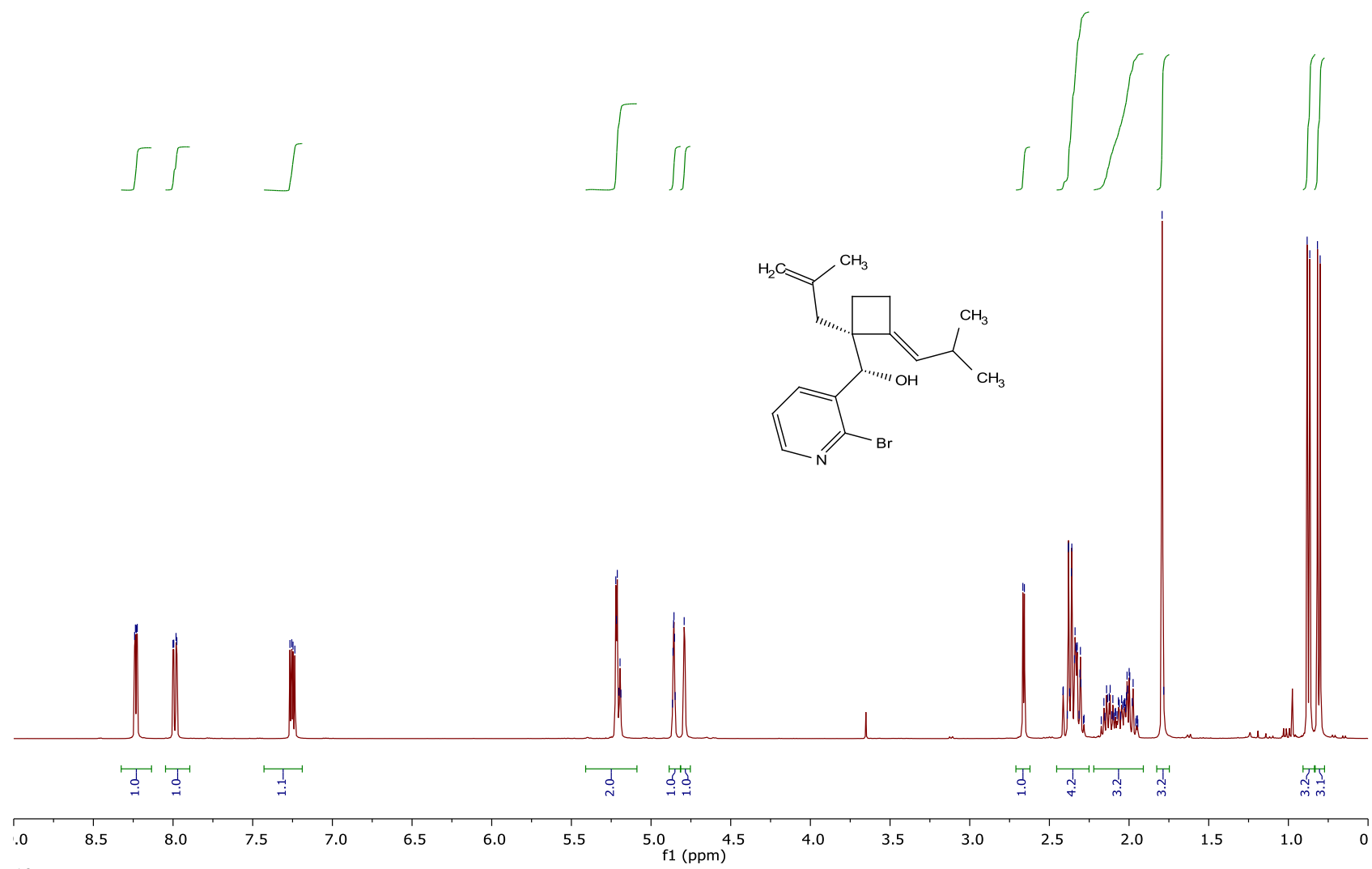

${ }^{13} \mathrm{C}$ NMR, $101 \mathrm{MHz}, \mathrm{CDCl}_{3}$

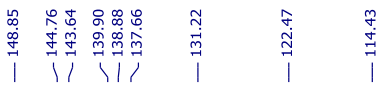

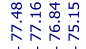

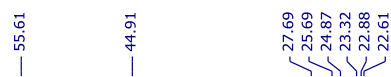

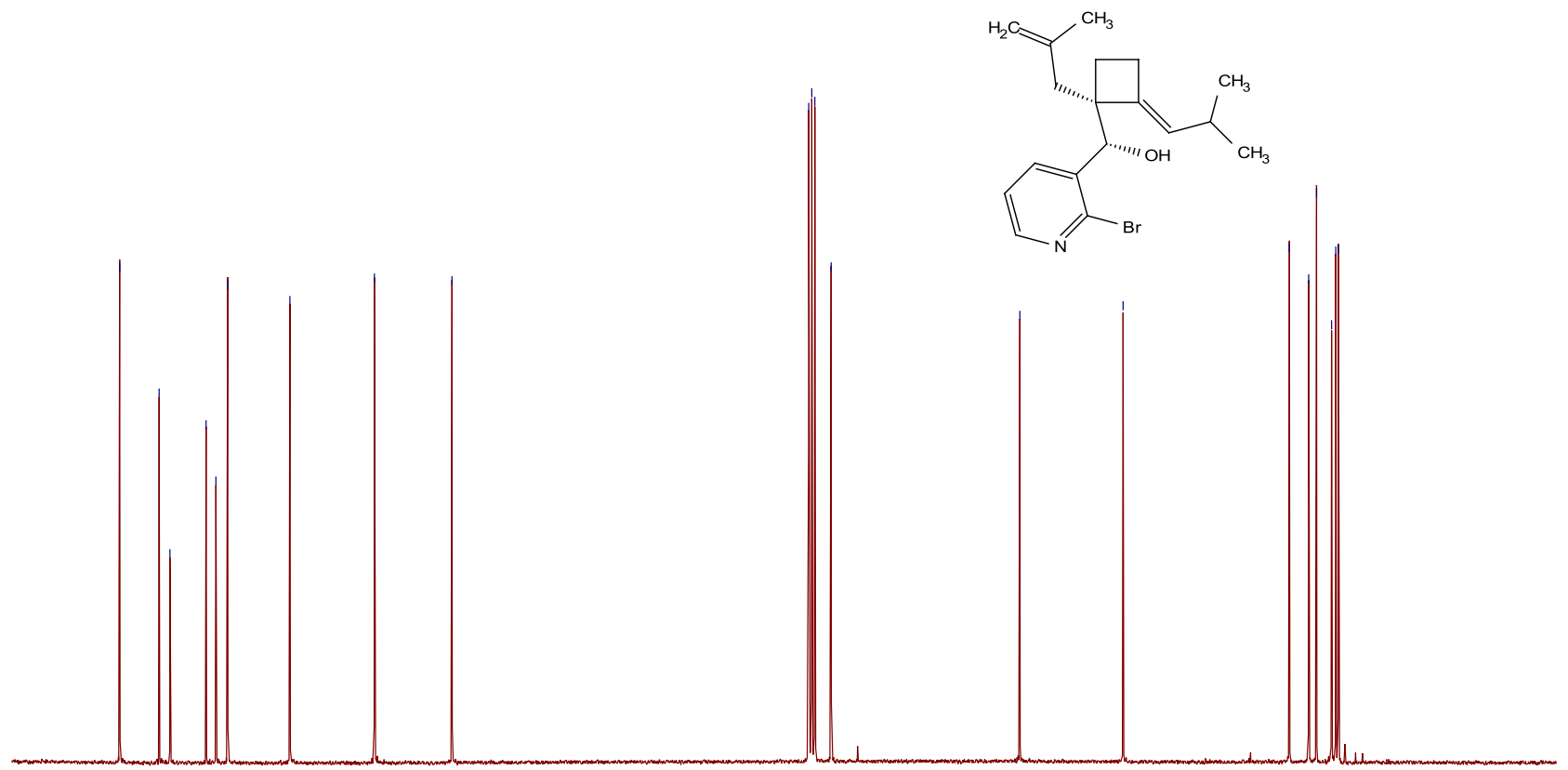

60

150

140

130

120

100

90

80
$\mathrm{f} 1(\mathrm{ppm})$ 


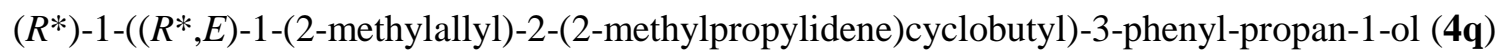

${ }^{1} \mathrm{H} \mathrm{NMR}, 400 \mathrm{MHz}, \mathrm{CDCl}_{3}$

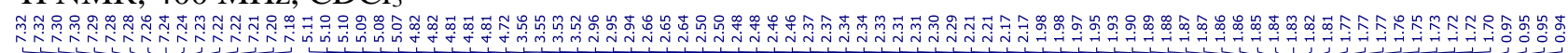
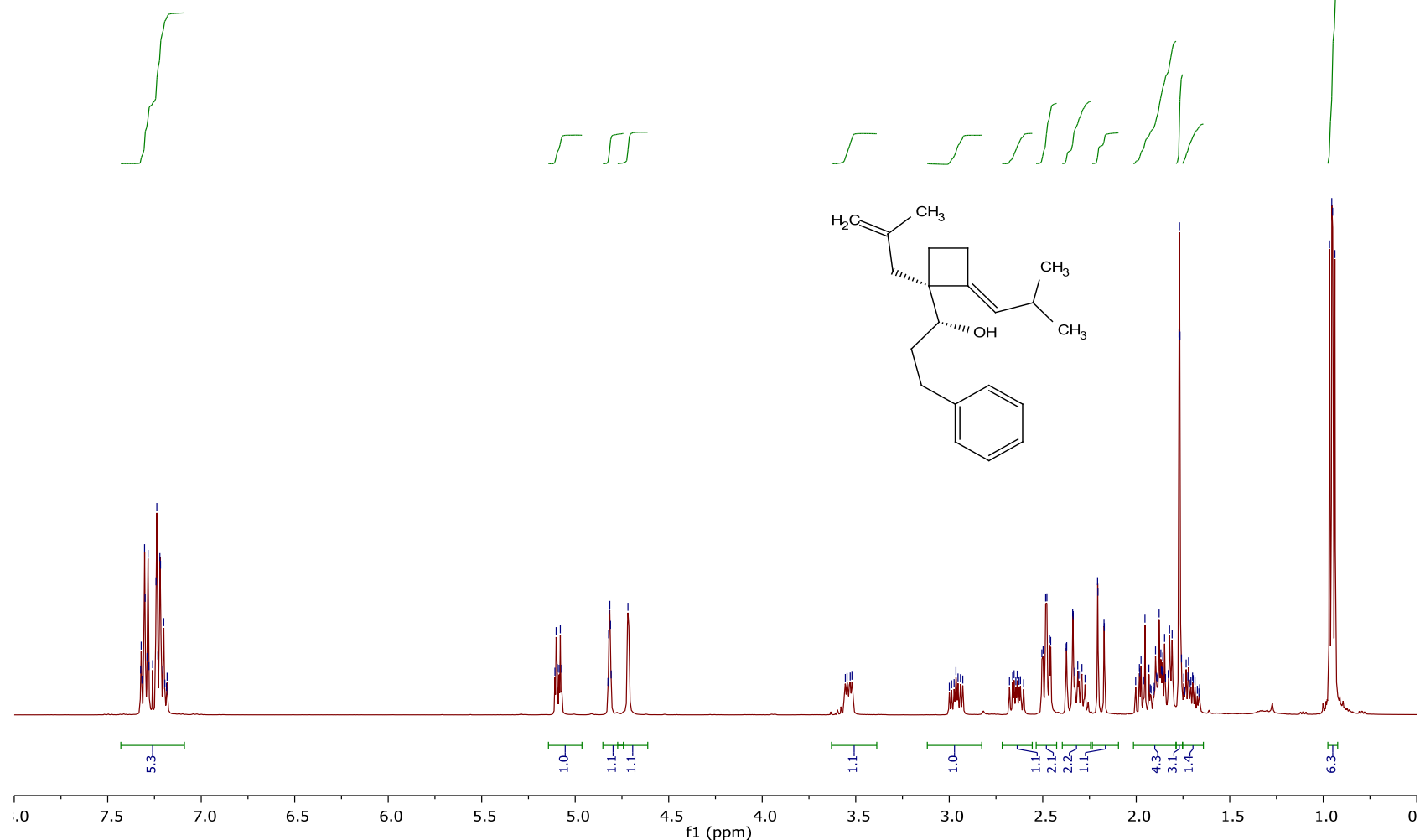

${ }^{13} \mathrm{C}$ NMR, $101 \mathrm{MHz}, \mathrm{CDCl}_{3}$

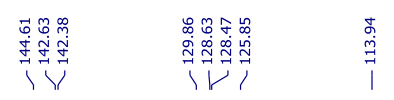

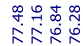

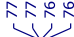

每
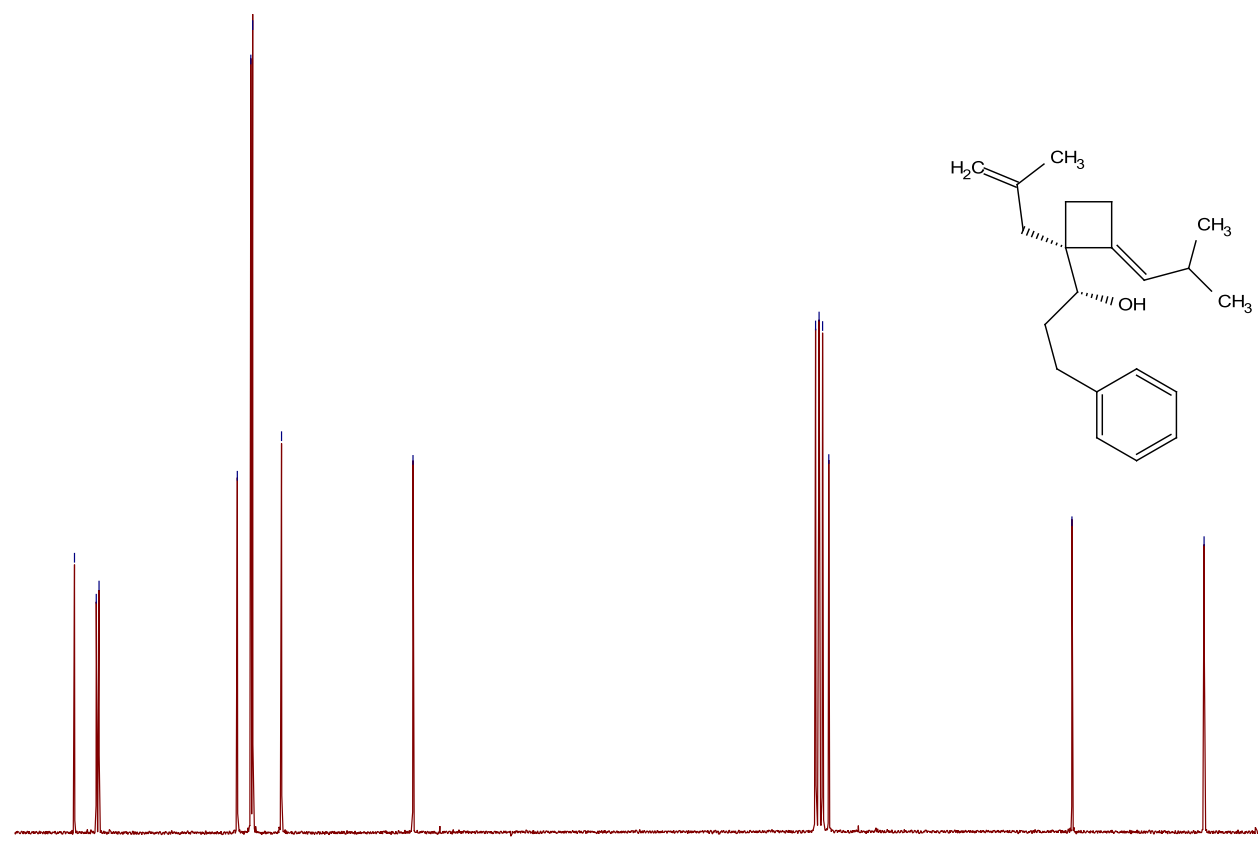

$\begin{array}{llllllllllllllll}50 & 145 & 140 & 135 & 130 & 125 & 120 & 115 & 110 & 105 & 100 & 95 & 90 & 85 & 80 & 75 \\ \mathrm{f} 1(\mathrm{ppm})\end{array}$ 
$\left(S^{*}\right)$-(3-bromo-2-iodo-4,5-dimethoxyphenyl)(( $\left.R^{*}, E\right)$-1-(2-methylallyl)-2-pentylidenecyclobutyl)methanol ${ }^{1} \mathrm{H} \mathrm{NMR}, 400 \mathrm{MHz}, \mathrm{CDCl}_{3}$

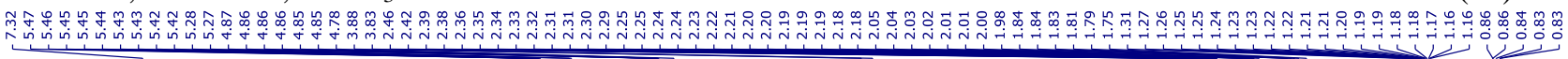

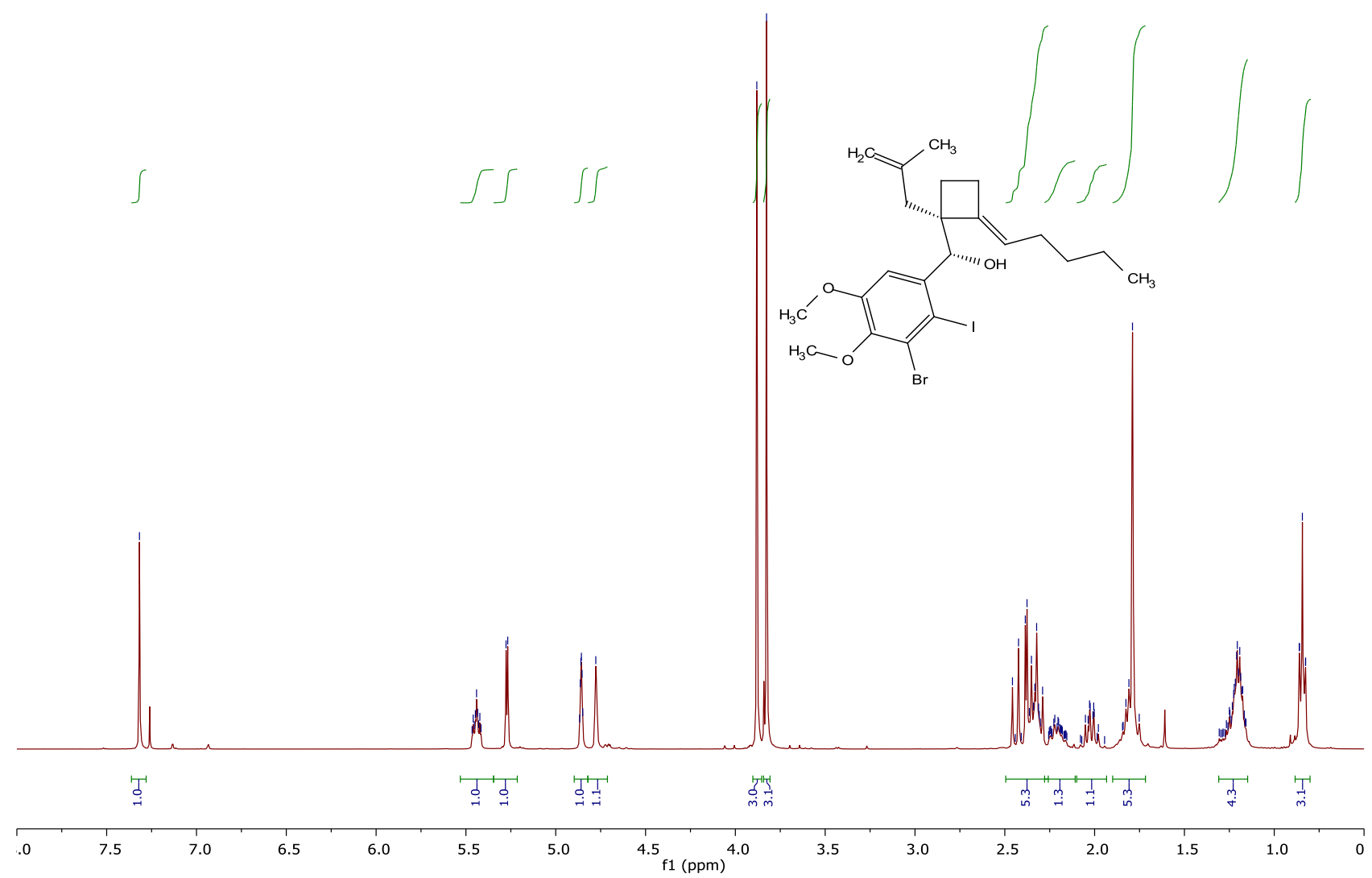

${ }^{13} \mathrm{C}$ NMR, $101 \mathrm{MHz}, \mathrm{CDCl}_{3}$

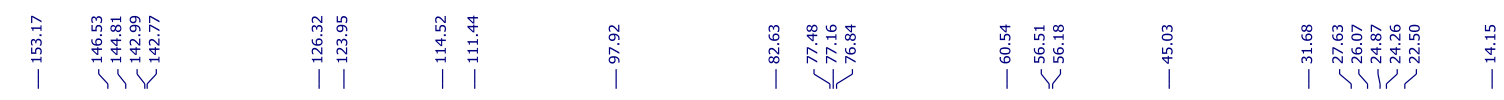

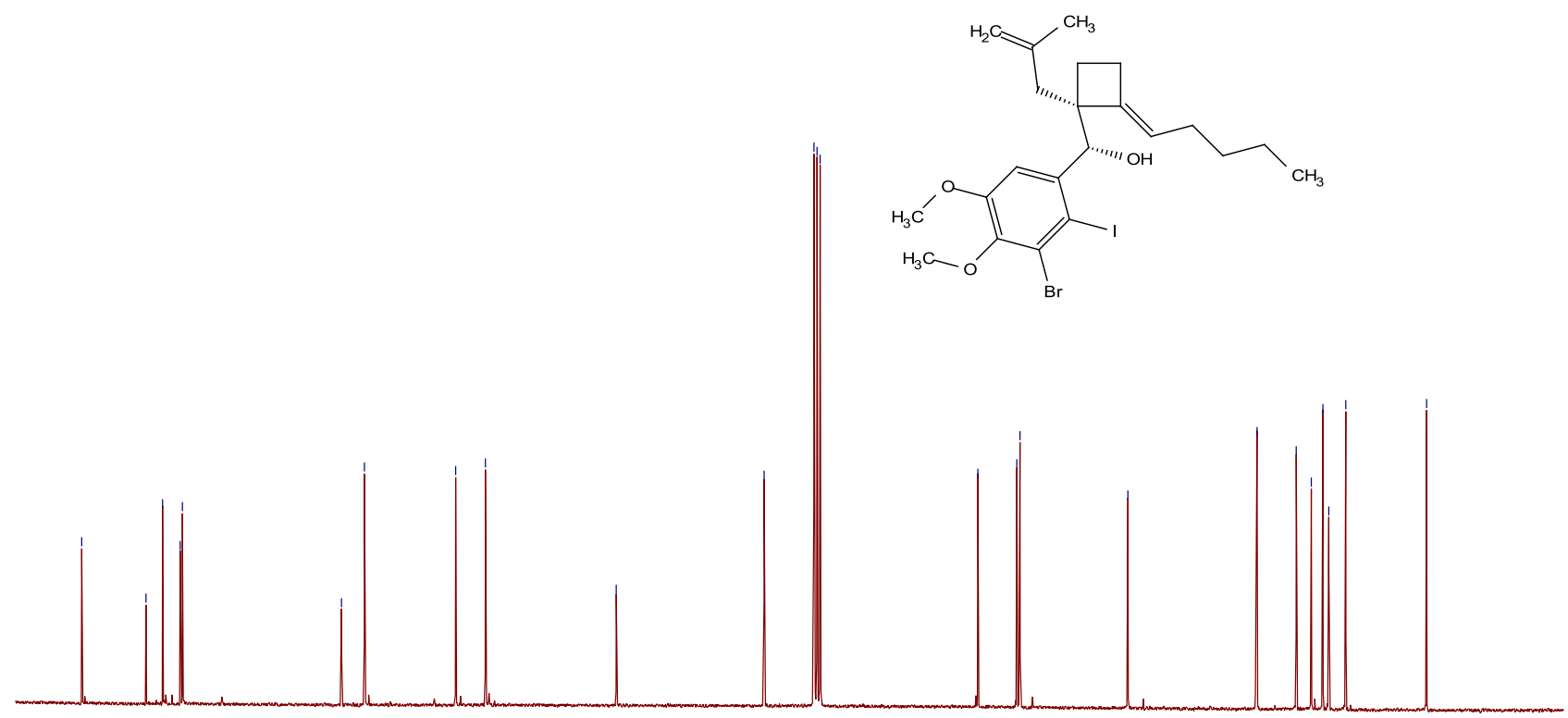

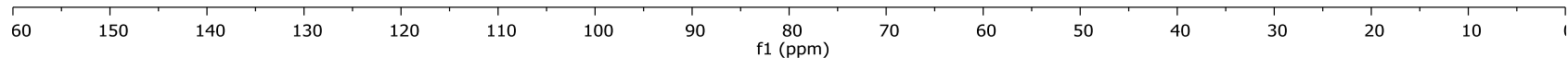


$\left(R^{*}\right)-\left(\left(R^{*}, E\right)-1-(2-m e t h y l a l l y l)-2-(3-p h e n y l p r o p y l i d e n e) c y c l o b u t y l\right)(p h e n y l) m e t h a n o l ~(4 \mathrm{~s})$

${ }^{1} \mathrm{H} \mathrm{NMR}, 400 \mathrm{MHz}, \mathrm{CDCl}_{3}$

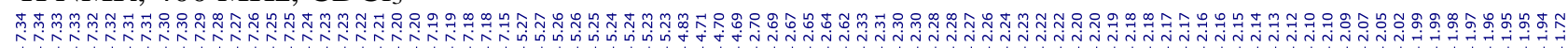
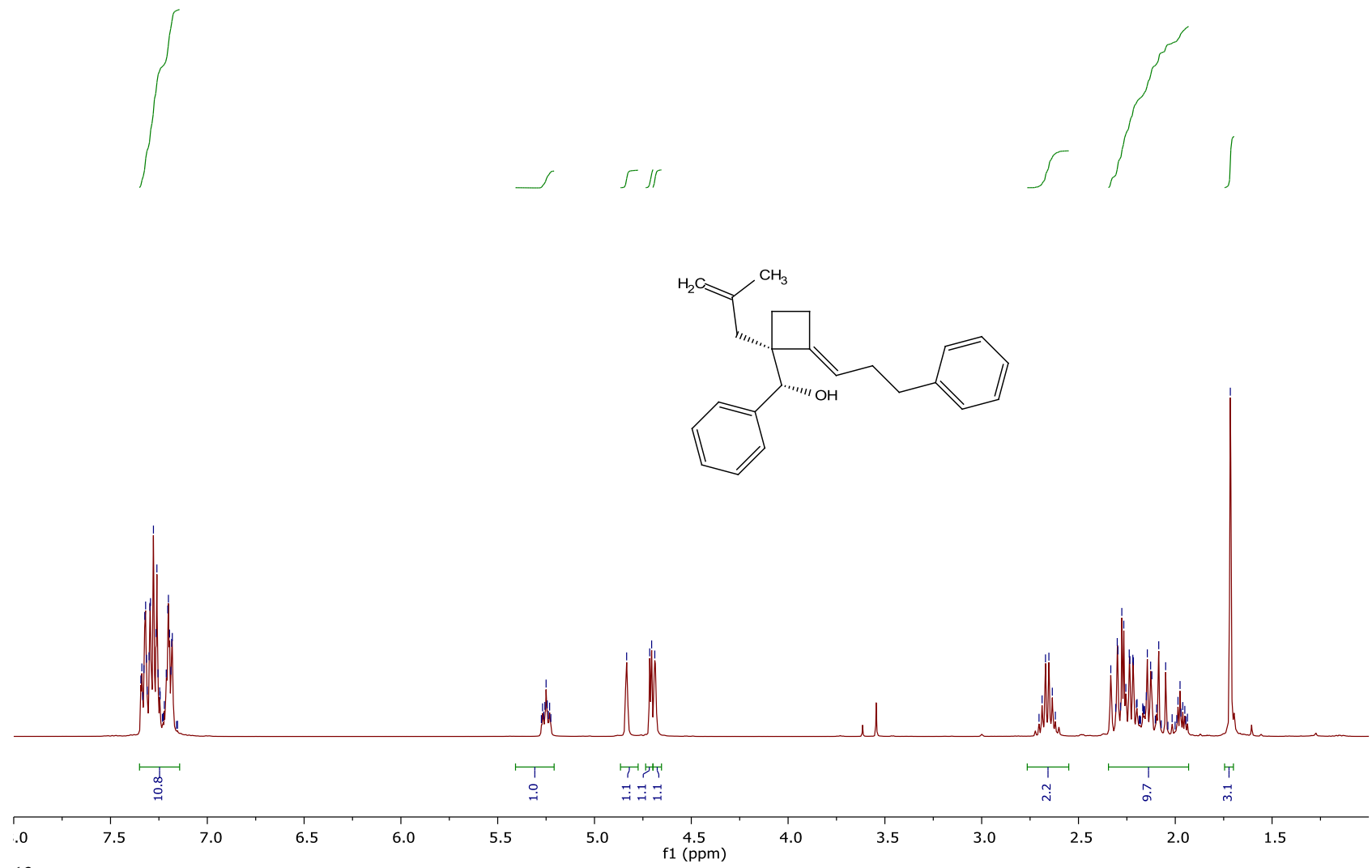

${ }^{13} \mathrm{C}$ NMR, $101 \mathrm{MHz}, \mathrm{CDCl}_{3}$

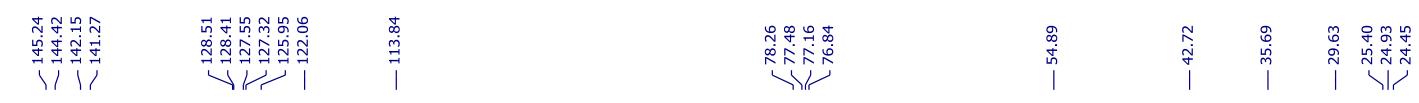

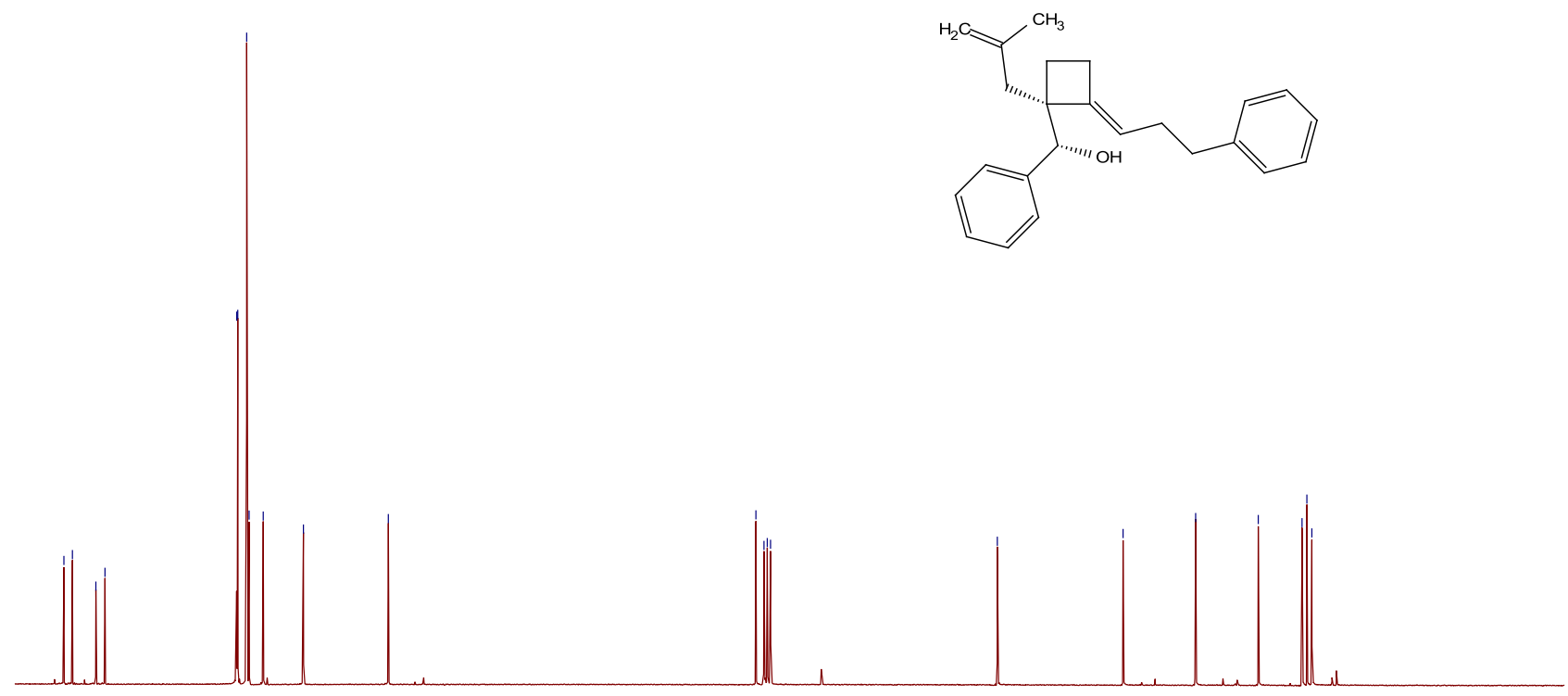

$\begin{array}{lllllllllllllllllllllllllllllllllllllllllllllll}50 & 145 & 140 & 135 & 130 & 125 & 120 & 115 & 110 & 105 & 100 & 95 & 90 & 85 & 80 & 75 & 70 & 65 & 60 & 55 & 50 & 45 & 40 & 35 & 30 & 25 & 20 & 15 & 10 & 5 & 15\end{array}$ 
$\left(S^{*}\right)-\left(\left(R^{*}, E\right)-2\right.$-(cyclopropylmethylene)-1-(2-methylallyl)cyclobutyl)(quinolin-3-yl)methanol (4t)

${ }^{1} \mathrm{H} \mathrm{NMR}, 400 \mathrm{MHz}, \mathrm{CDCl}_{3}$

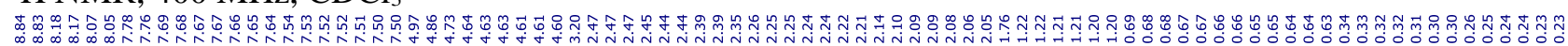

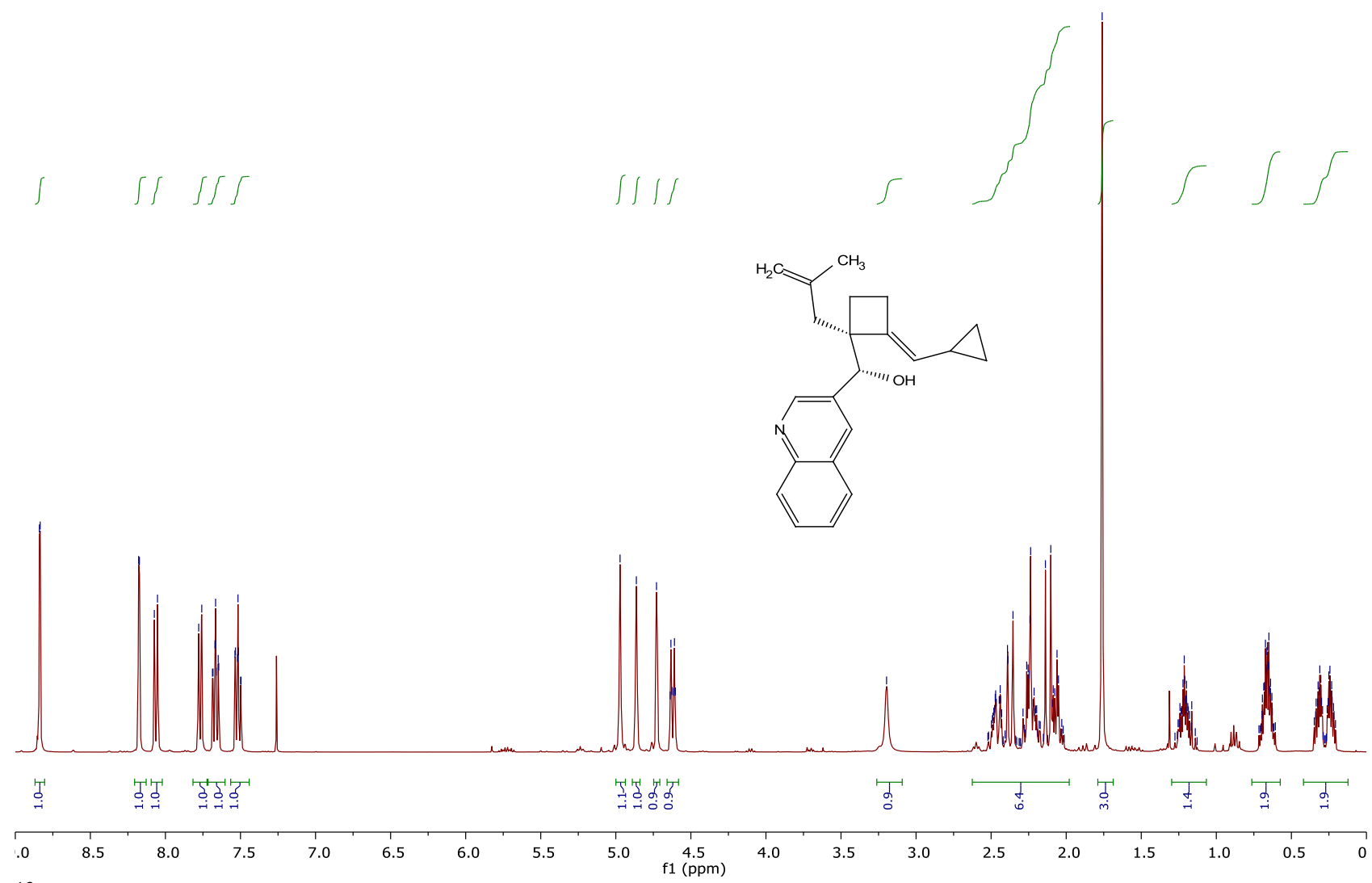

${ }^{13} \mathrm{C}$ NMR, $101 \mathrm{MHz}, \mathrm{CDCl}_{3}$

|j

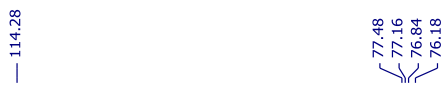

$\underbrace{1}$

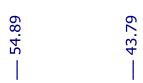

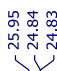

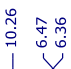

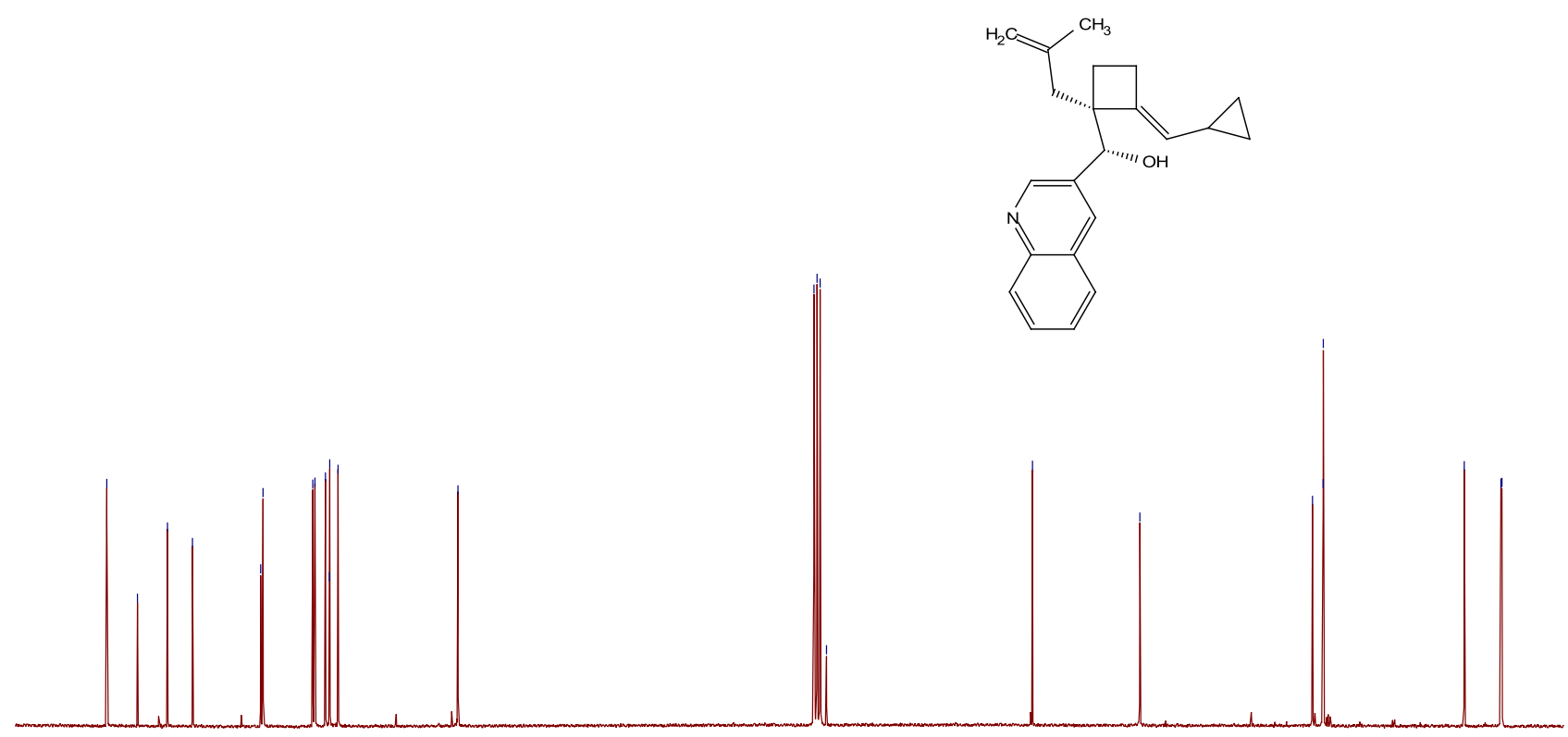

60

$50 \quad 140$

$130 \quad 120 \quad 110$

100

90 80
$1 \mathrm{ppm})$ 
$\left(R^{*}\right)-\left(\left(R^{*}, E\right)-2-e t h y l i d e n e-1-(2-m e t h y l a l l y l) c y c l o b u t y l\right)($ phenyl)methanol (4u)

${ }^{1} \mathrm{H} \mathrm{NMR}, 400 \mathrm{MHz}, \mathrm{CDCl}_{3}$

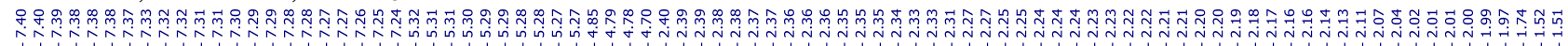
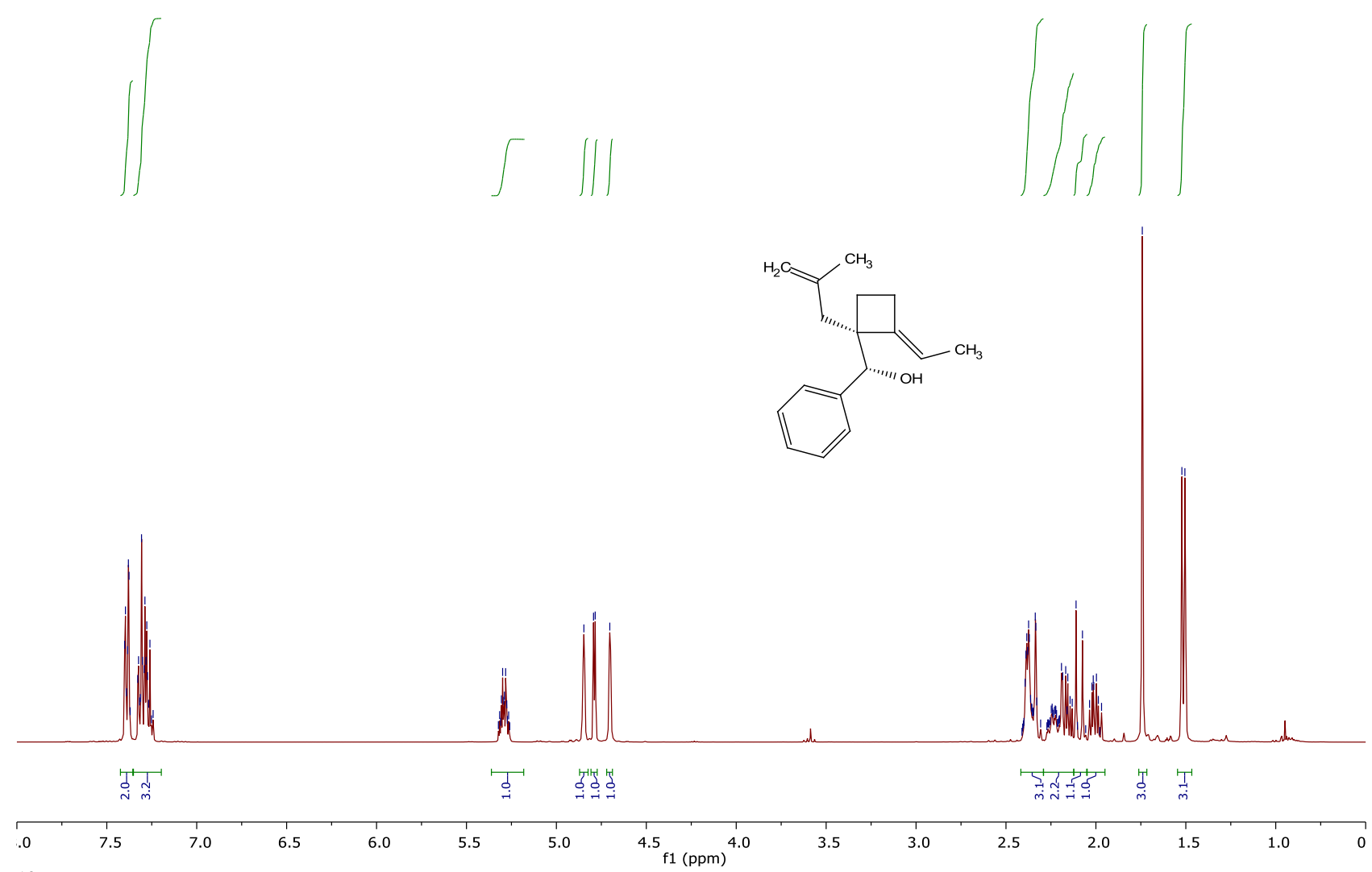

${ }^{13} \mathrm{C}$ NMR, $101 \mathrm{MHz}, \mathrm{CDCl}_{3}$

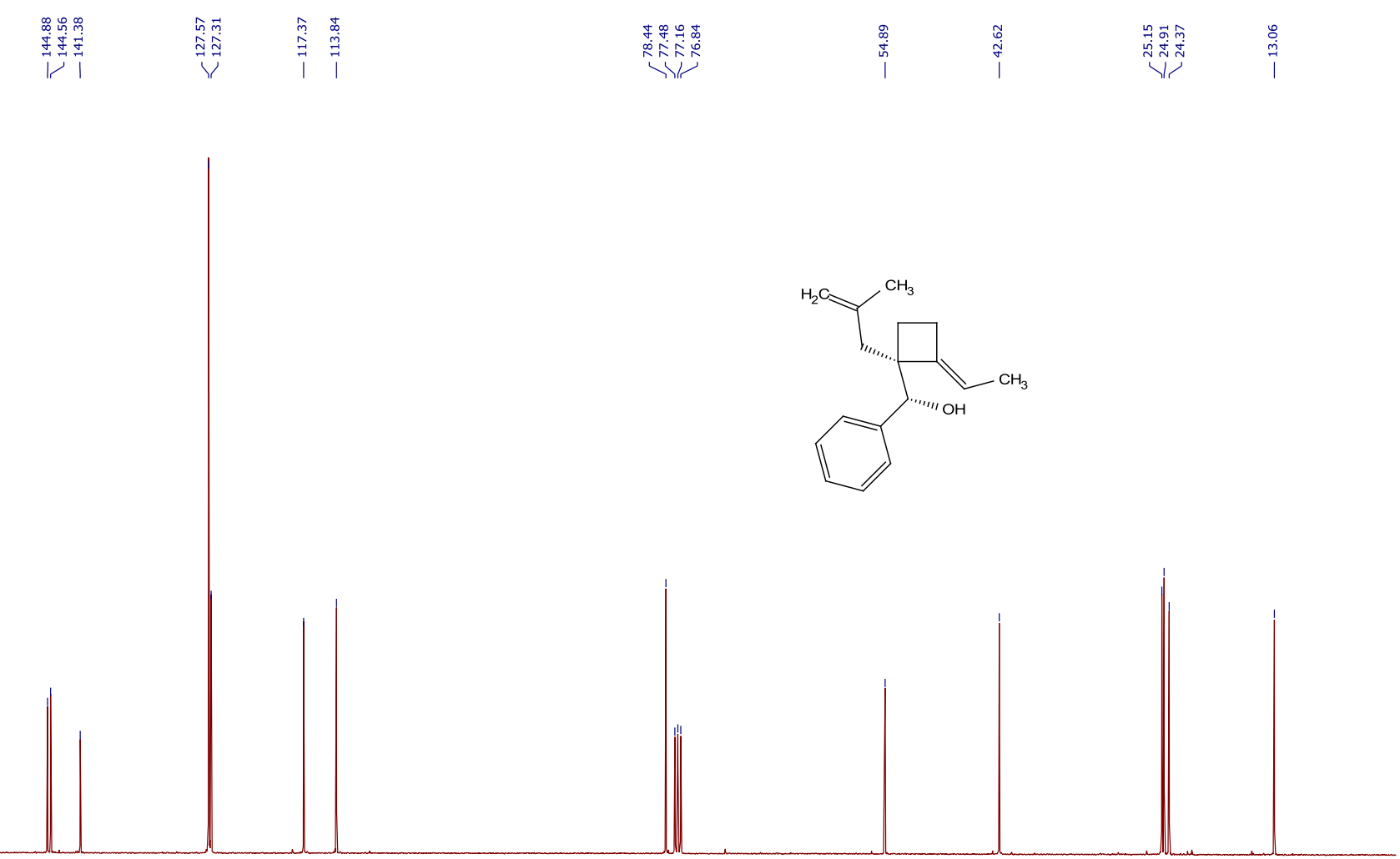

$\begin{array}{lllllllllllllllllllllllllllllllllll}50 & 145 & 140 & 135 & 130 & 125 & 120 & 115 & 110 & 105 & 100 & 95 & 90 & 85 & 80 & 75 & 70 & 65 & 60 & 55 & 50 & 45 & 40 & 35 & 30 & 25 & 20 & 15 & 10 & 5 & 1\end{array}$ 
$\left(S^{*}\right)$-(2-bromopyridin-3-yl) $\left(\left(S^{*}, E\right)\right.$-1-methyl-2-pentylidenecyclobutyl)methanol (4v)

${ }^{1} \mathrm{H} \mathrm{NMR}, 400 \mathrm{MHz}, \mathrm{CDCl}_{3}$

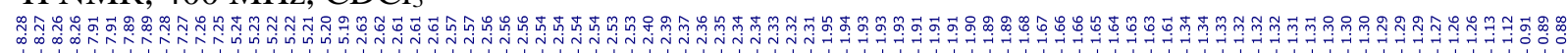
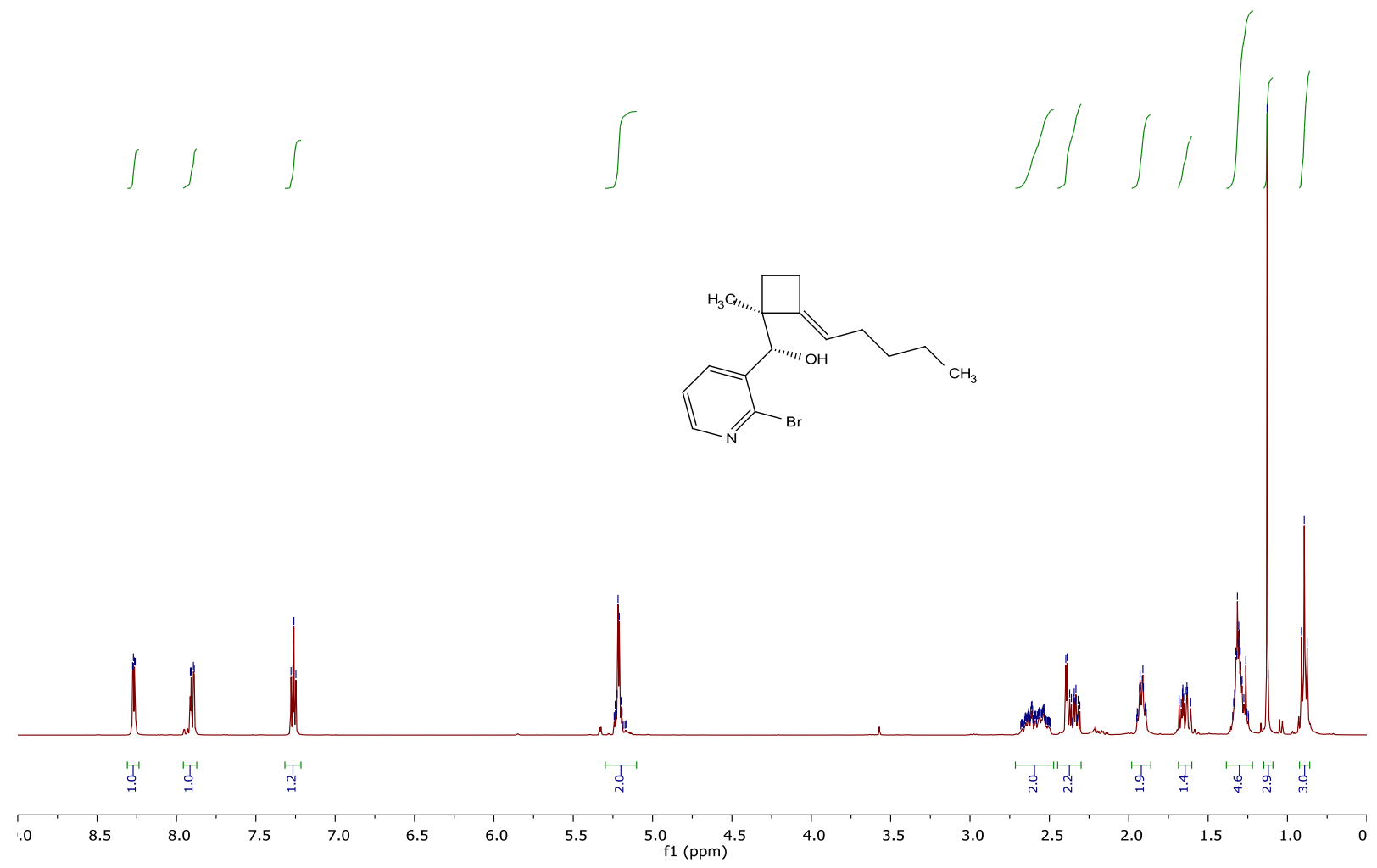

${ }^{13} \mathrm{C} \mathrm{NMR}, 101 \mathrm{MHz}, \mathrm{CDCl}_{3}$

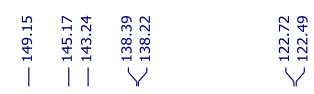

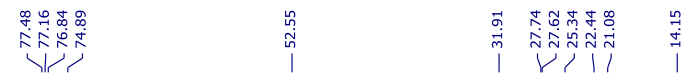

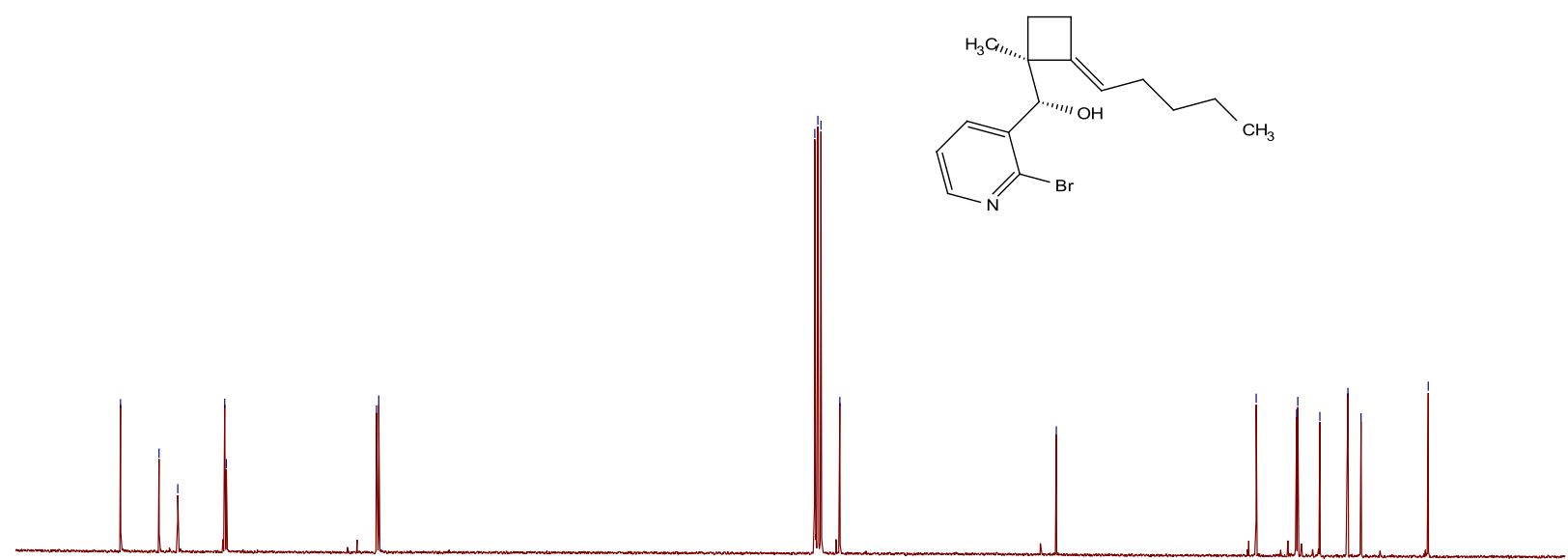

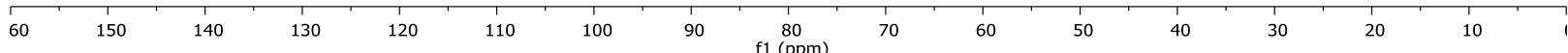


$\left(R^{*}\right)$-1-(( $\left.R^{*}, E\right)$-1-allyl-2-ethylidenecyclobutyl)-3-phenylpropan-1-ol (4w)

${ }^{1} \mathrm{H} \mathrm{NMR}, 400 \mathrm{MHz}, \mathrm{CDCl}_{3}$

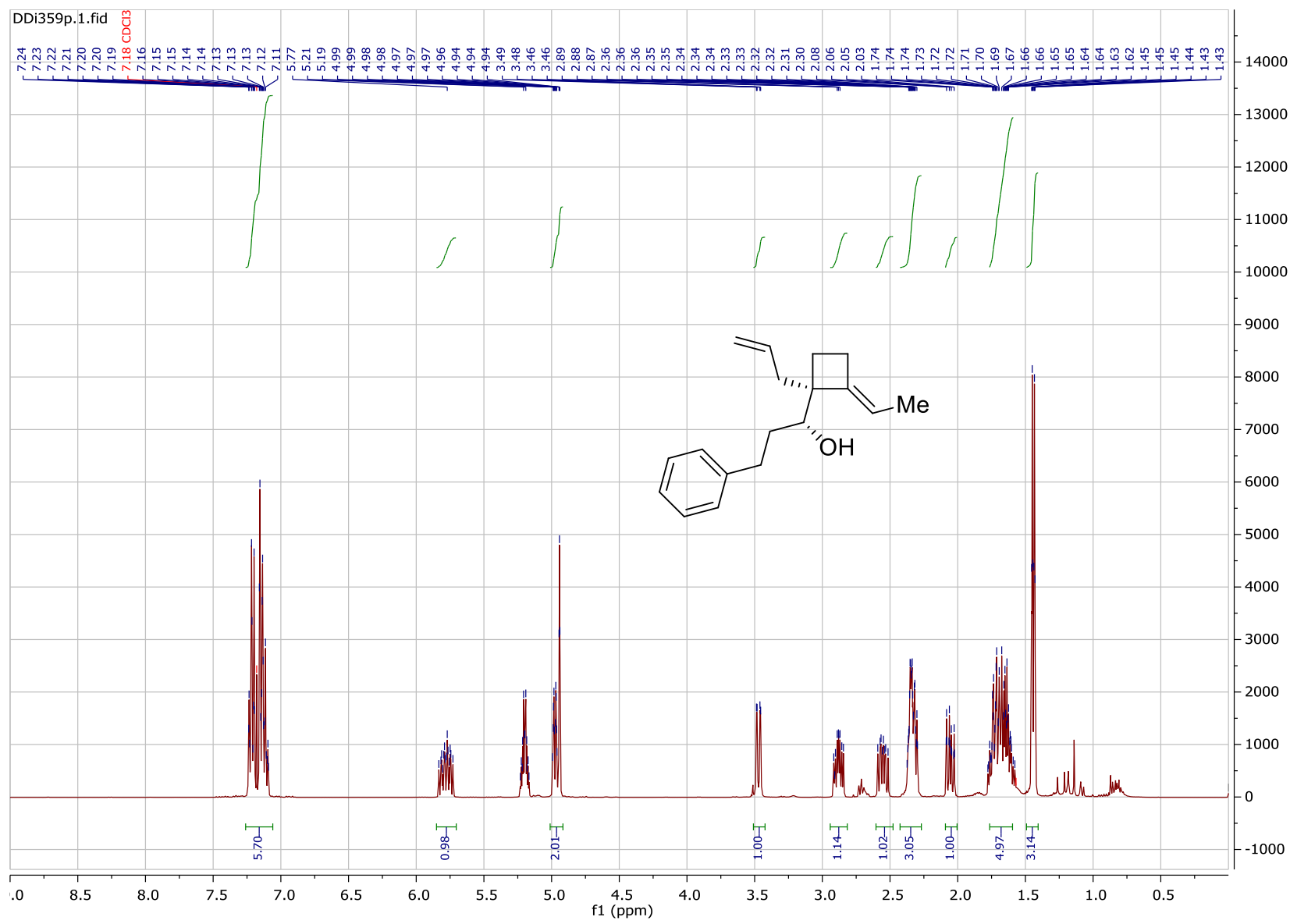

${ }^{13} \mathrm{C}$ NMR, $101 \mathrm{MHz}, \mathrm{CDCl}_{3}$

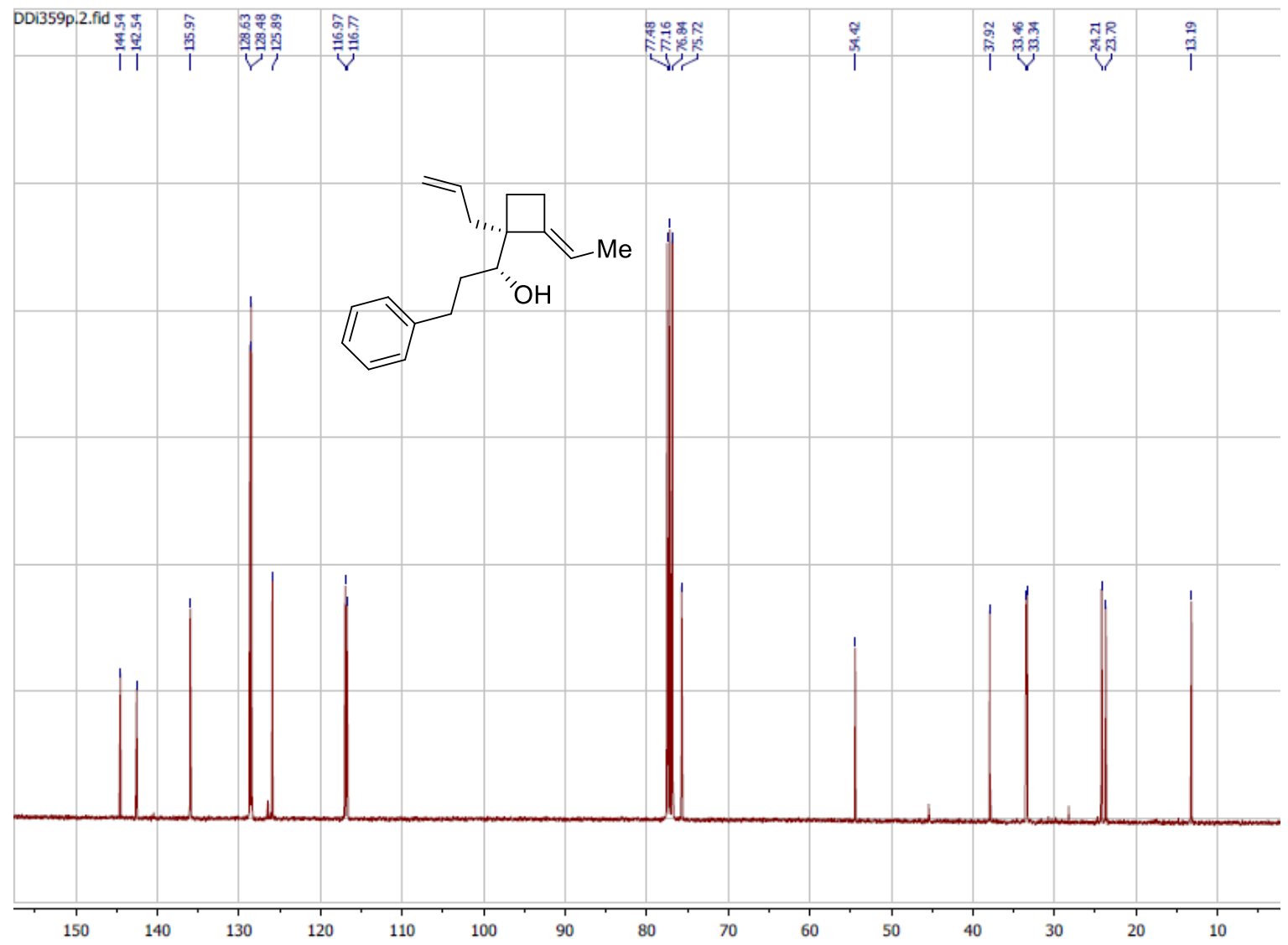




\section{Procedures for the enantioselective sequence}

\section{a) Experimental data}<smiles>C=C(C)CC1([C@H](O)c2cc(OC)c(OC)c(Br)c2I)CCC1=CCCC</smiles>

(S)-(3-bromo-2-iodo-4,5-dimethoxyphenyl)((R,E)-1-(2-methylallyl)2-pentylidenecyclobutyl)methanol ((-)4r)

Using (2-methylallyl)zinc bromide as the zinc organyl, (4R,5R)-2-butyl-4,5-dicyclohexyl-1,3,2dioxaborolane and 3-bromo-2-iodo-4,5-dimethoxybenzaldehyde according to general procedure $\mathbf{D}$, provided $(-) 4 \mathbf{r}(170 \mathrm{mg}, 62 \%)$ as a colourless oil in 99:1 $d r$ and $99 \%$ ee.

$[\alpha]_{D}^{19}=-41.33^{\circ}\left(\mathrm{c}=1.25 ; \mathrm{CH}_{2} \mathrm{Cl}_{2}\right)$.

$(-) 4 \mathbf{r}$ was also obtained following general procedure $\mathbf{C}$, with equal results.<smiles>C=C(C)CC1(C(O)c2cc(OC)c(OC)c(Br)c2I)CCC1=CCCC</smiles>

$(R)$-(3-bromo-2-iodo-4,5-dimethoxyphenyl)((S,E)-1-(2-methylallyl)2-pentylidenecyclobutyl)methanol ((+)4r)

Using (2-methylallyl)zinc bromide as the zinc organyl, $n$-butyllithium as the lithium species, (1S,2S)-1,2dicyclohexylethane-1,2-diol, diisopropyl (dichloromethyl)boronate and 3-bromo-2-iodo-4,5dimethoxybenzaldehyde according to general procedure $\mathbf{C}$, provided $(+) 4 \mathbf{r}(152 \mathrm{mg}, 55 \%)$ as a colourless oil in 99:1 $d r$ and $99 \%$ ee.

$[\alpha]_{D}^{19}=+36.73^{\circ}\left(\mathrm{c}=0.98 ; \mathrm{CH}_{2} \mathrm{Cl}_{2}\right)$.

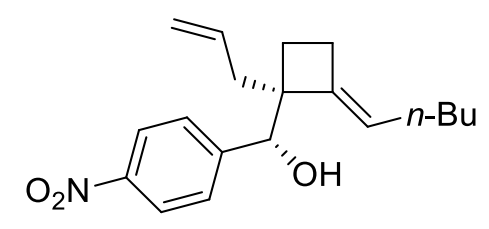

$(R)-((R, E)-1$-allyl-2-pentylidenecyclobutyl)(4-nitrophenyl)methanol $((+) \mathbf{4 l})$

Using allylzinc bromide as the zinc organyl, $n$-butyllithium as the lithium species, $\quad(1 R, 2 R)$-1,2-dicyclohexylethane-1,2-diol, diisopropyl (dichloromethyl)boronate and 4nitrobenzaldehyde according to general procedure $\mathbf{C}$, provided (+)4l (91 mg, 58\%) as white crystals in 99:1 $d r$ and $99 \%$ ee .

$[\alpha]_{D}^{19}=+2.00^{\circ}\left(\mathrm{c}=1.00 ; \mathrm{CH}_{2} \mathrm{Cl}_{2}\right)$. 


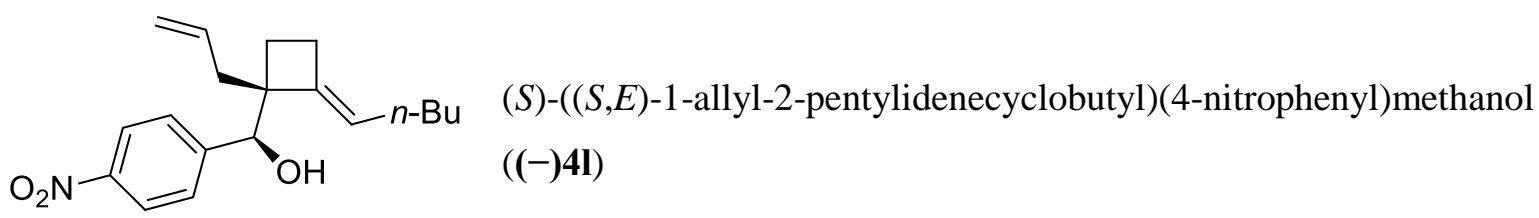

Using allylzinc bromide as the zinc organyl, $n$-butyllithium as the lithium species, (1S,2S)-1,2dicyclohexylethane-1,2-diol, diisopropyl (dichloromethyl)boronate and 4-nitrobenzaldehyde according to general procedure $\mathbf{C}$, provided (-)4l (74 $\mathrm{mg}, 47 \%)$ as white crystals in 99:1 $\mathrm{dr}$ and 99\% ee.

$[\alpha]_{D}^{19}=-23.16^{\circ}\left(\mathrm{c}=0.57 ; \mathrm{CH}_{2} \mathrm{Cl}_{2}\right)$.

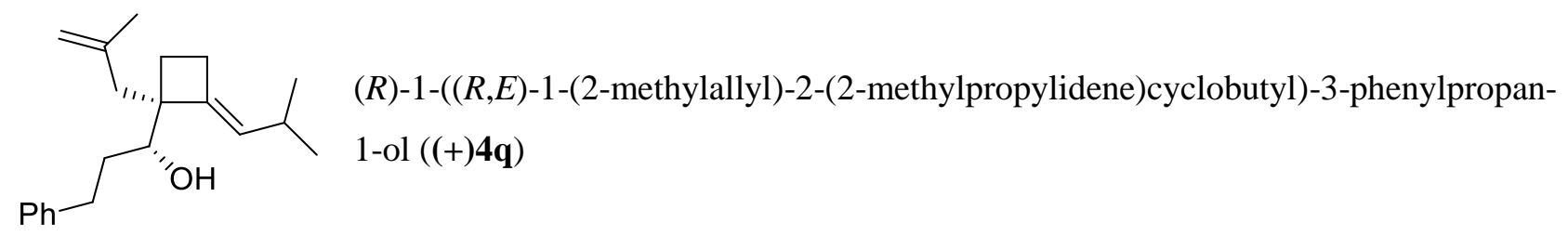

Using (2-methylallyl)zinc bromide as the zinc organyl, iso-propylmagnesium chloride as the magnesium species, (1R,2R)-1,2-dicyclohexylethane-1,2-diol, diisopropyl (dichloromethyl)boronate and 3phenylpropanal according to general procedure $\mathbf{C}$, provided $(+) \mathbf{4 q}(86 \mathrm{mg}, 58 \%)$ as a colourless oil in 99:1 $d r$ and $99 \%$ ee.

$[\alpha]_{D}^{19}=+31.51^{\circ}\left(\mathrm{c}=0.86 ; \mathrm{CH}_{2} \mathrm{Cl}_{2}\right)$.

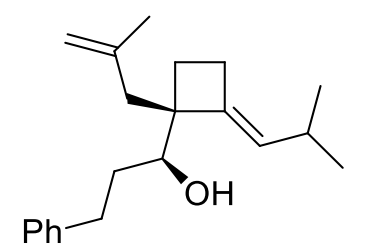

(S)-1-((S,E)-1-(2-methylallyl)-2-(2-methylpropylidene)cyclobutyl)-3-phenylpropan$1-\mathrm{ol}((-) \mathbf{4 q})$

Using (2-methylallyl)zinc bromide as the zinc organyl, iso-propylmagnesium chloride as the magnesium species, (1S,2S)-1,2-dicyclohexylethane-1,2-diol, diisopropyl (dichloromethyl)boronate and 3phenylpropanal according to general procedure $\mathbf{C}$, provided (-)4q (118 $\mathbf{m g}, 79 \%)$ as a colourless oil in 99:1 $d r$ and $99 \%$ ee.

$[\alpha]_{D}^{19}=-24.63^{\circ}\left(\mathrm{c}=1.34 ; \mathrm{CH}_{2} \mathrm{Cl}_{2}\right)$. 
<smiles>C=C(C)C[C@]1([C@H](O)c2cccnc2Br)CCC1=CC(C)C</smiles>

$(S)$-(2-bromopyridin-3-yl)((R,E)-1-(2-methylallyl)-2-(2methylpropylidene)cyclobutyl)methanol $((-) 4 p)$

Using (2-methylallyl)zinc bromide as the zinc organyl, iso-propylmagnesium chloride as the magnesium species, $\quad(1 R, 2 R)$-1,2-dicyclohexylethane-1,2-diol, diisopropyl (dichloromethyl)boronate and 2bromonicotinaldehyde according to general procedure $\mathbf{C}$, provided (-)4p (159 $\mathrm{mg}, 91 \%)$ as a colourless oil in 99:1 $d r$ and 97\% ee.

$[\alpha]_{D}^{19}=-48.40^{\circ}\left(\mathrm{c}=1.00 ; \mathrm{CH}_{2} \mathrm{Cl}_{2}\right)$.<smiles>C=C(C)CC1(C(O)c2cccnc2Br)CCC1=CC(C)C</smiles>

$(R)$-(2-bromopyridin-3-yl)((S,E)-1-(2-methylallyl)-2-(2methylpropylidene)cyclobutyl)methanol ((+)4p)

Using (2-methylallyl)zinc bromide as the zinc organyl, iso-propylmagnesium chloride as the magnesium species, (1S,2S)-1,2-dicyclohexylethane-1,2-diol, diisopropyl (dichloromethyl)boronate and 2bromonicotinaldehyde according to general procedure $\mathbf{C}$, provided $(+) \mathbf{4 p}(154 \mathrm{mg}, 88 \%)$ as a colourless oil in 99:1 $d r$ and 97\% ee.

$[\alpha]_{D}^{19}=+46.40^{\circ}\left(\mathrm{c}=1.00 ; \mathrm{CH}_{2} \mathrm{Cl}_{2}\right)$. 


\section{b) Chiral HPLC chromatograms}

OF; $10 \%$ iPrOH in Heptane; $0.5 \mathrm{~mL} / \mathrm{min} ; 25^{\circ} \mathrm{C}$
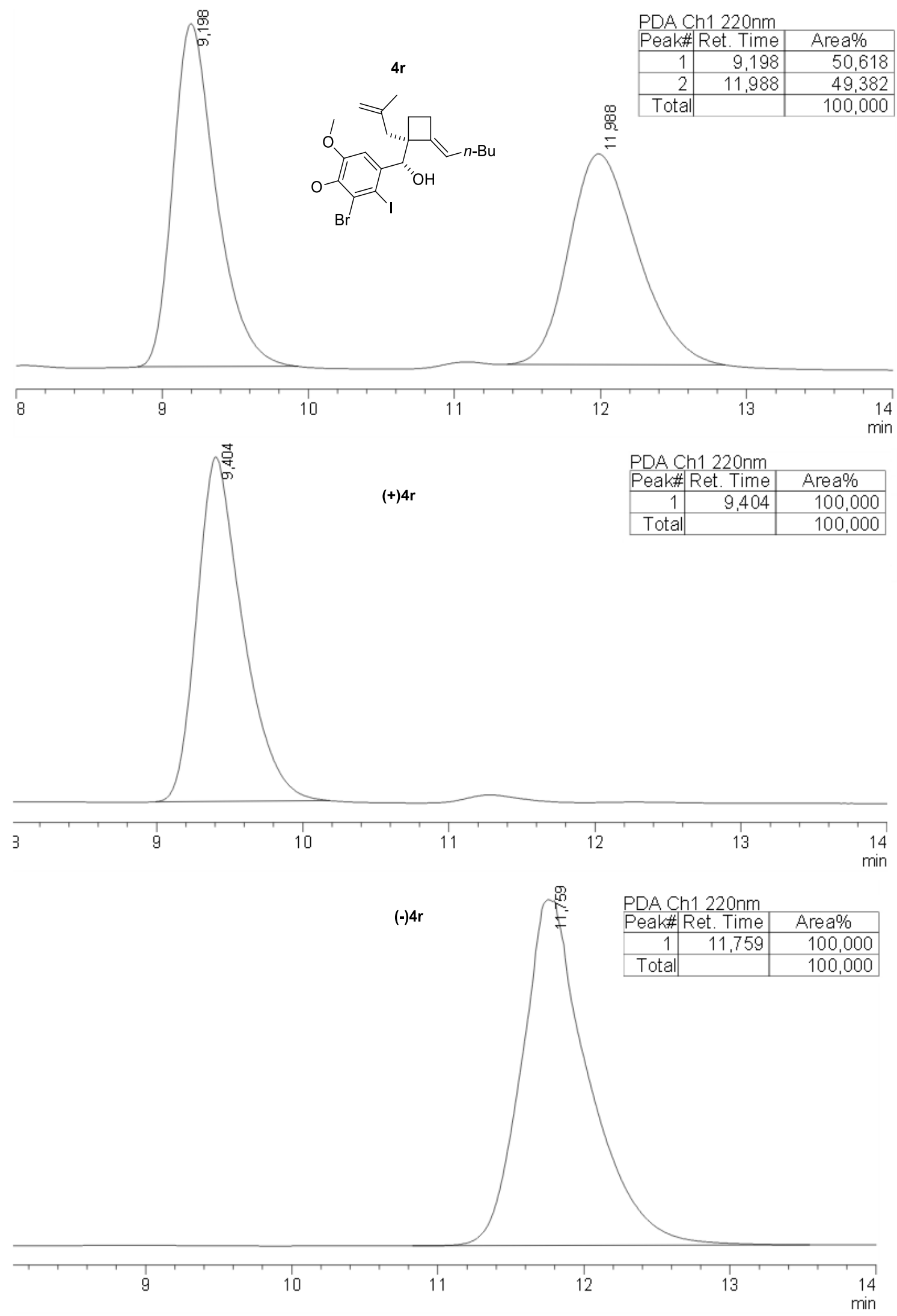

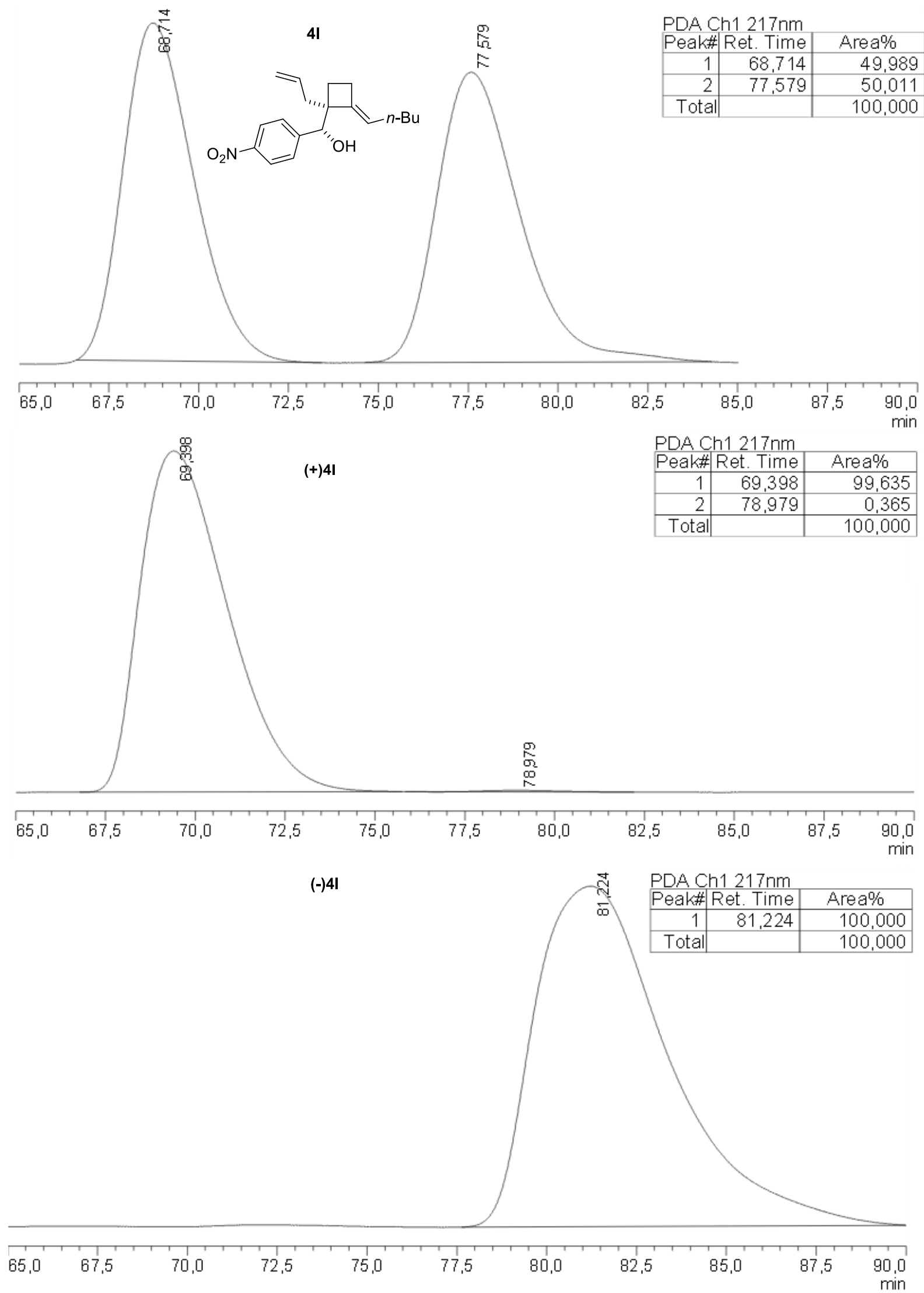


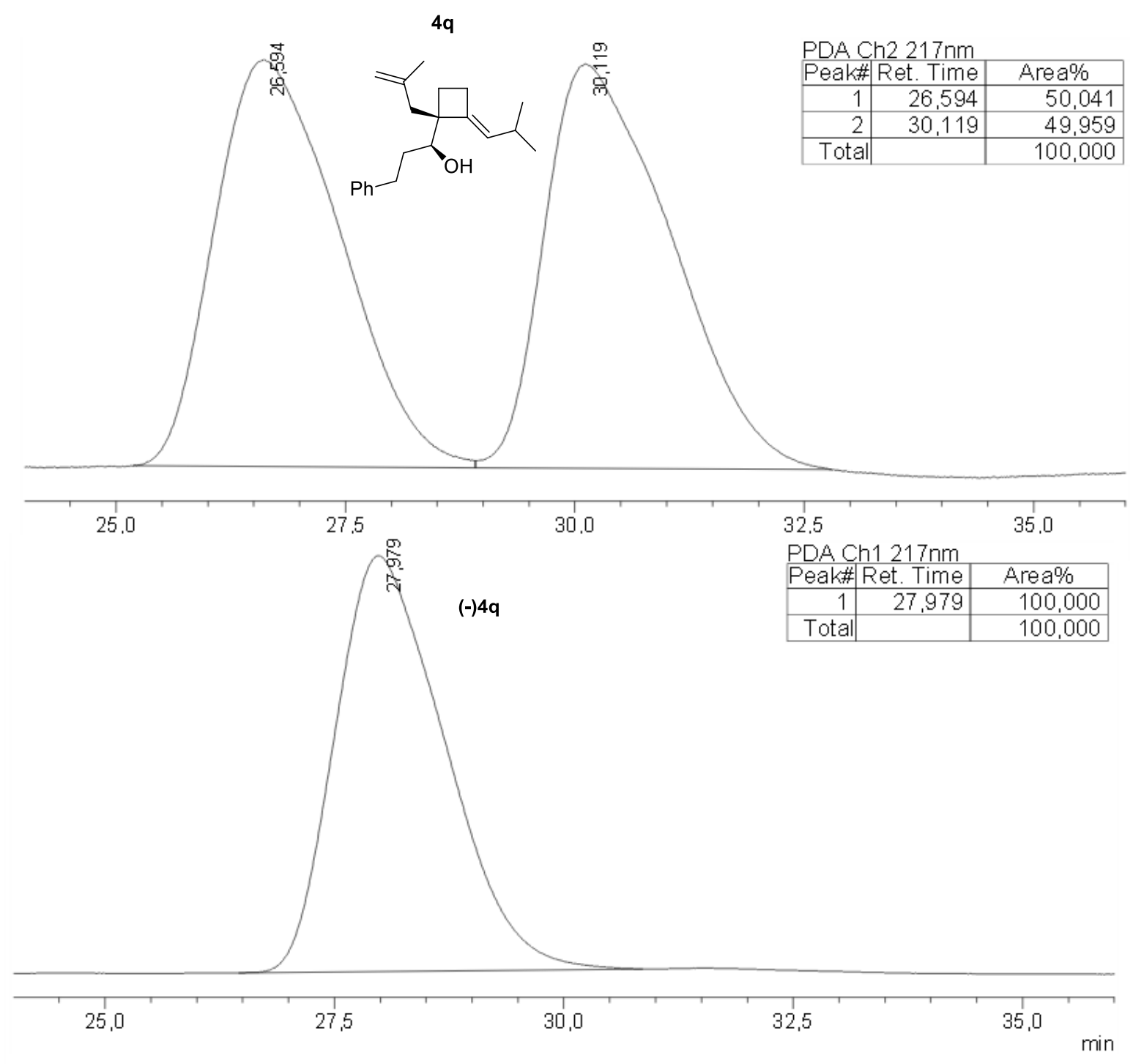

$(+) 4 q$

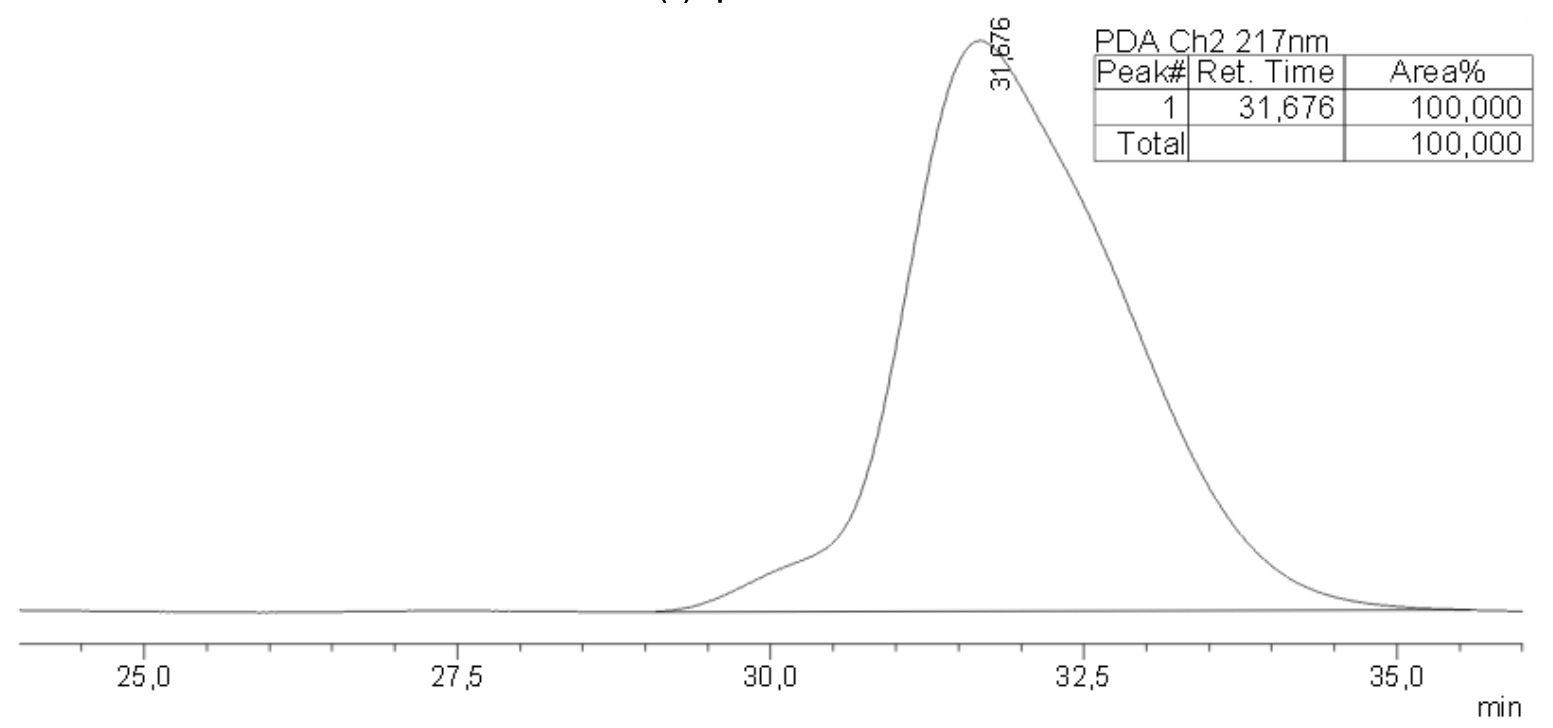



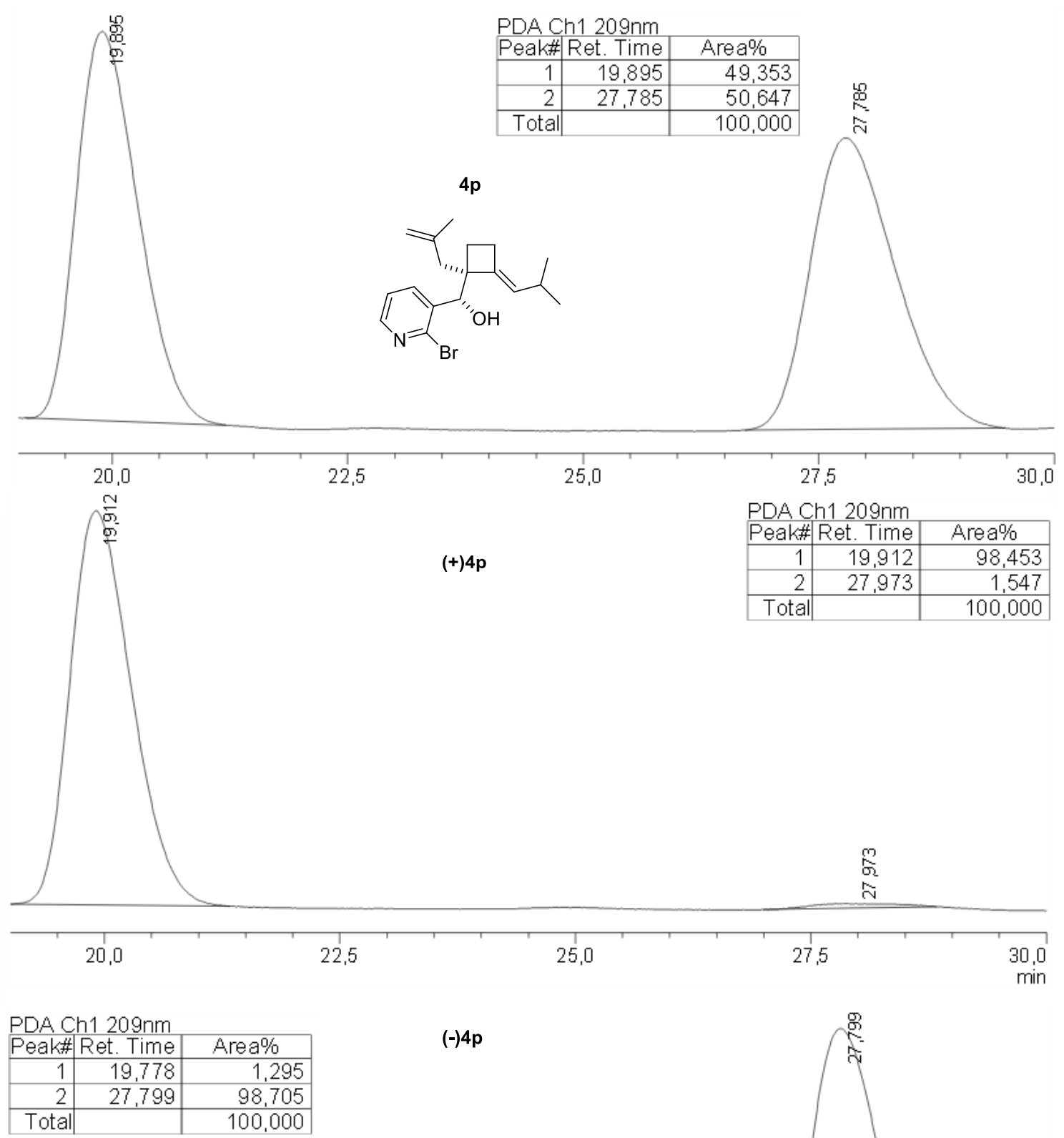

$(-) 4 p$

\begin{tabular}{|c|c|c|}
\hline Peak\# & Ret. Time & Area\% \\
\hline 1 & 19.778 & 1,295 \\
\hline 2 & 27.799 & 98,705 \\
\hline Tota & & 100,000 \\
\hline
\end{tabular}

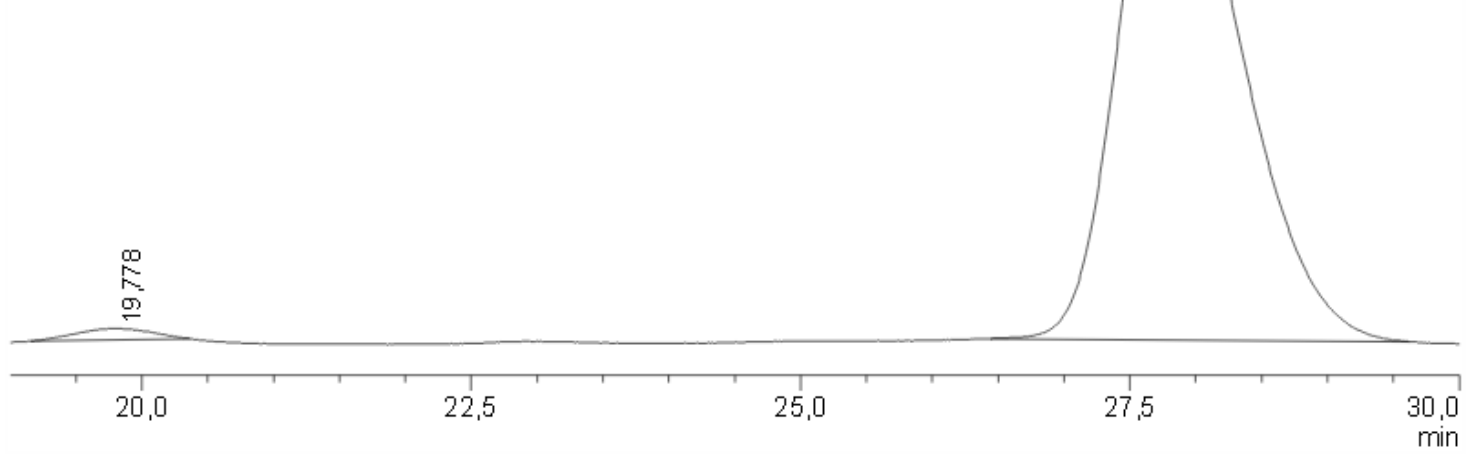




\section{Representative chromatograms for diastereoisomeric ratios determination}

Gas chromatogram of $\mathbf{4 a}$

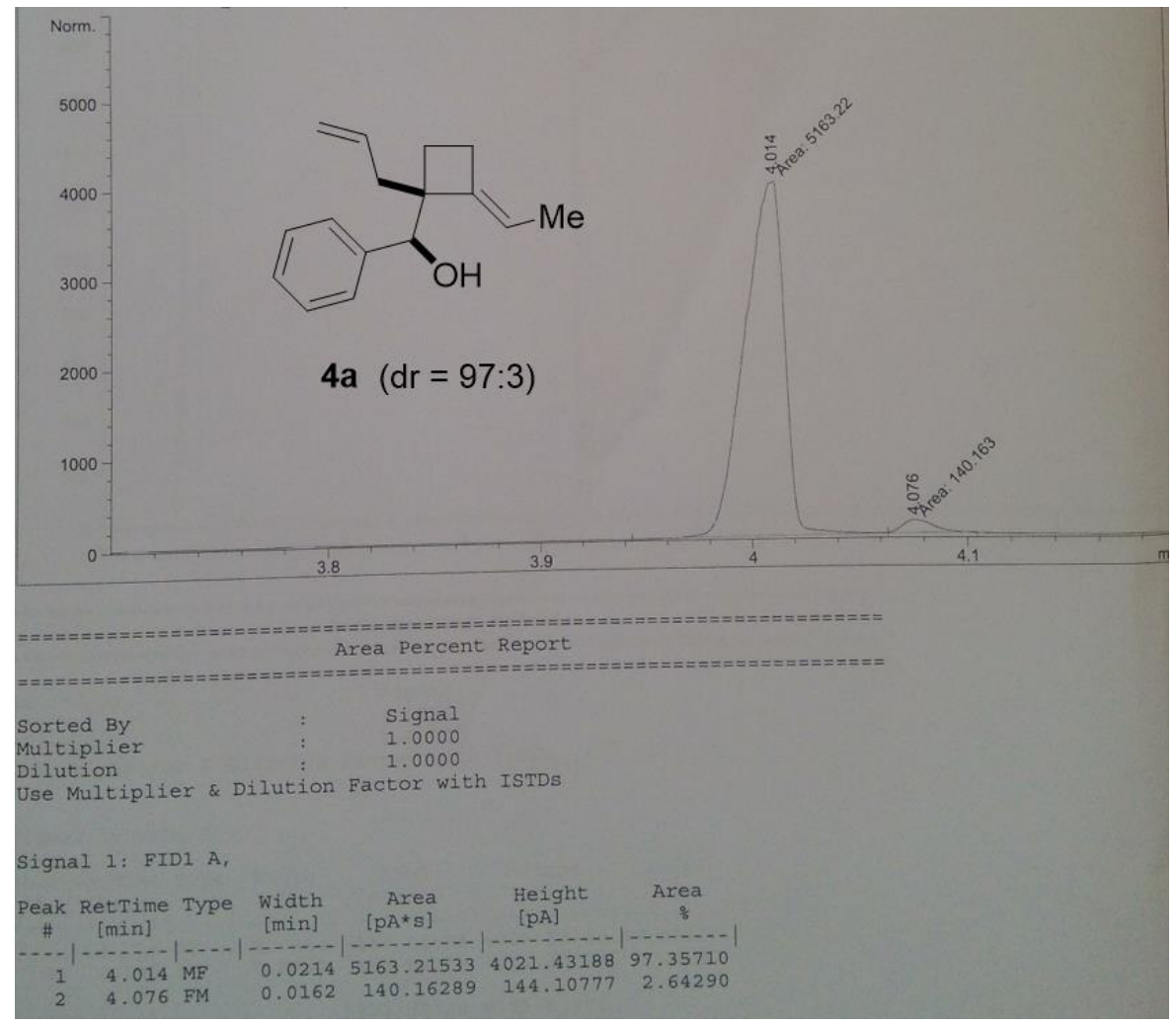

Gas chromatogram of $\mathbf{4 f}$

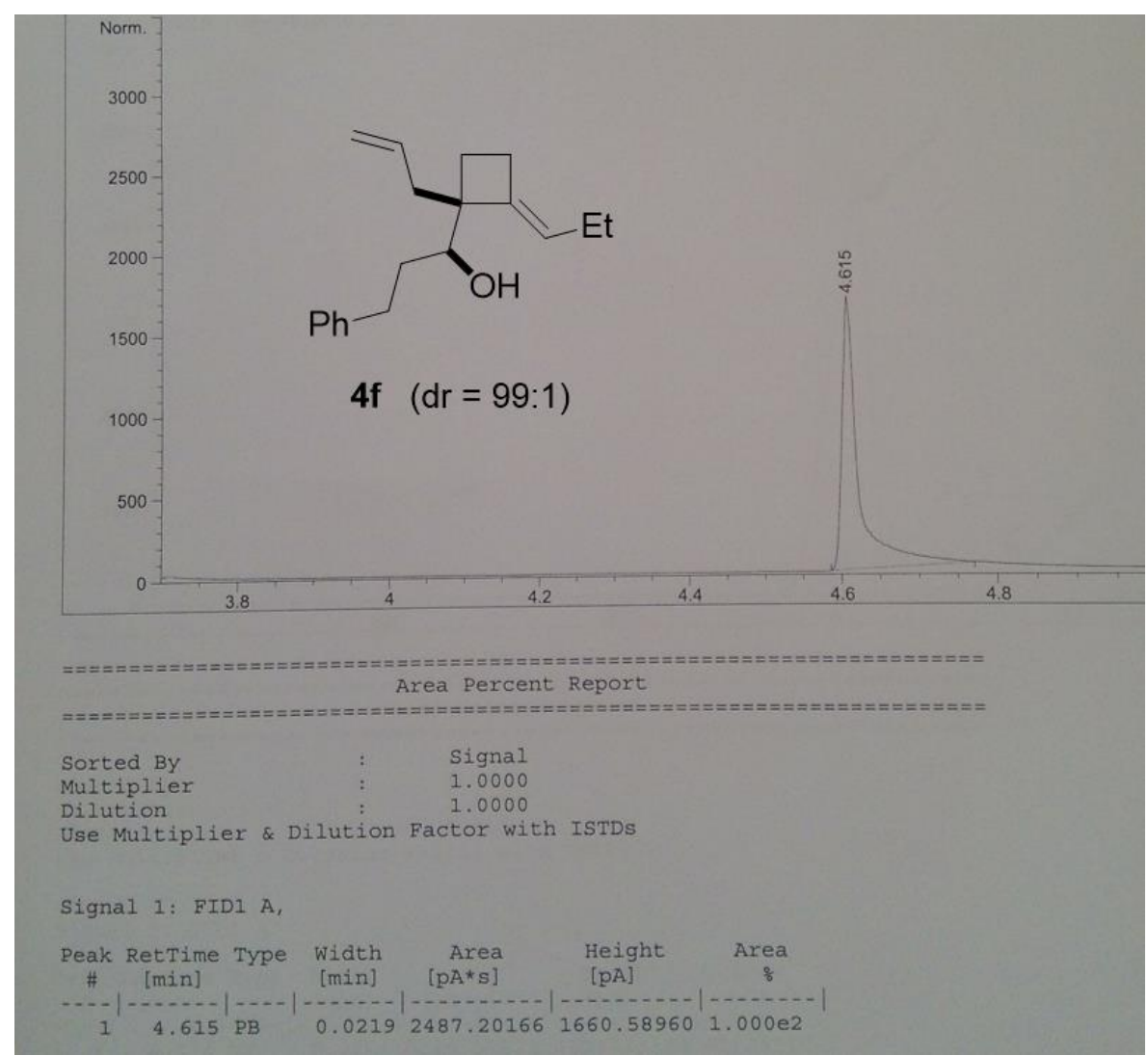

